of

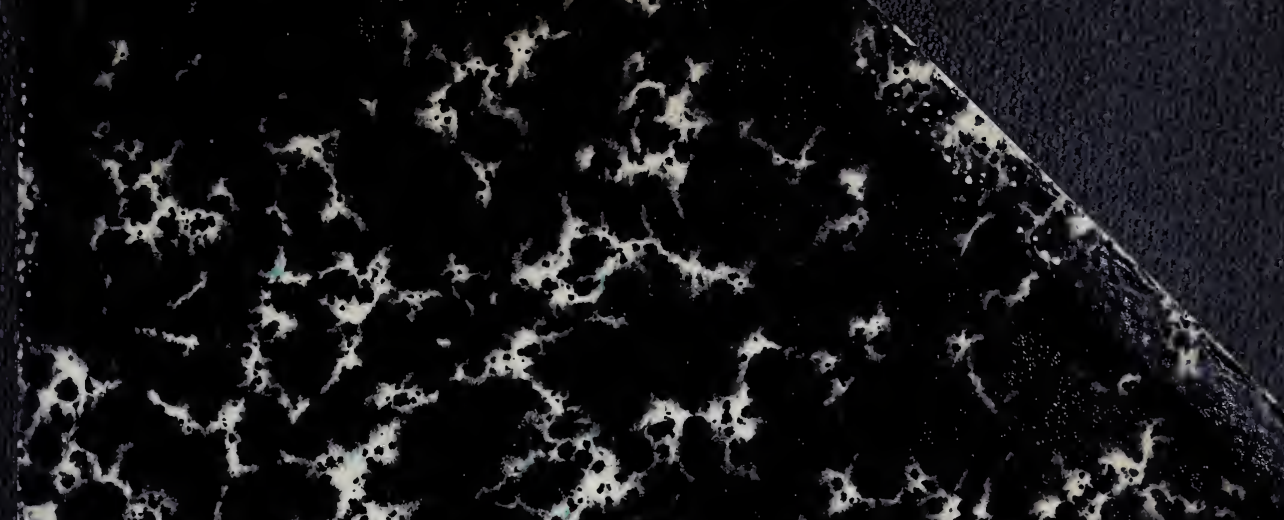

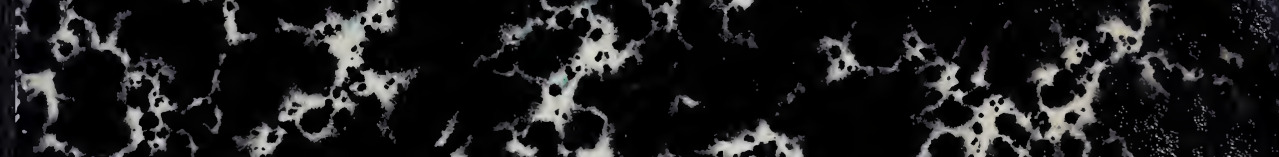

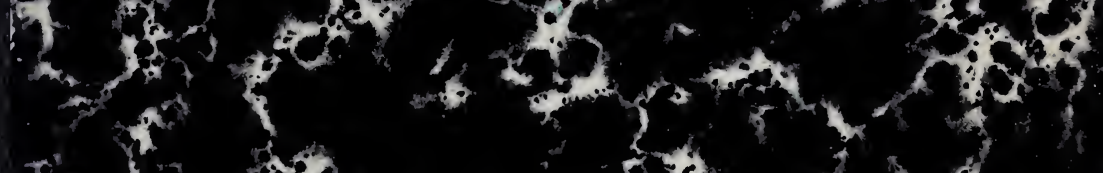

1. *

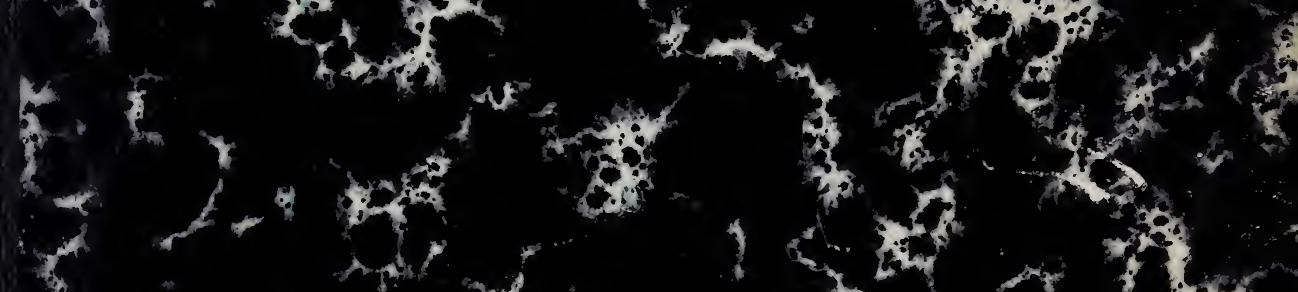

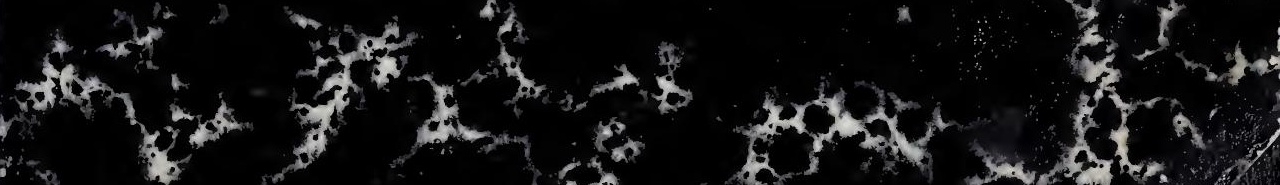

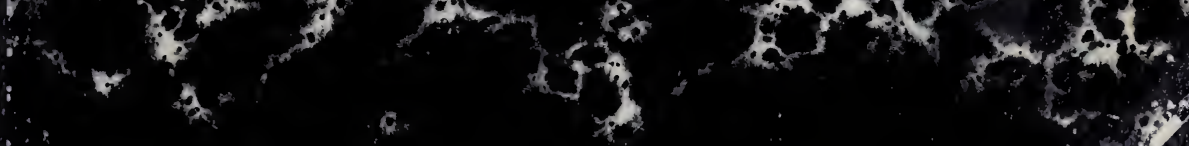

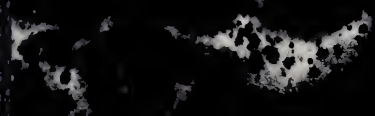




$$
\begin{gathered}
R 3453 \\
\times .2
\end{gathered}
$$






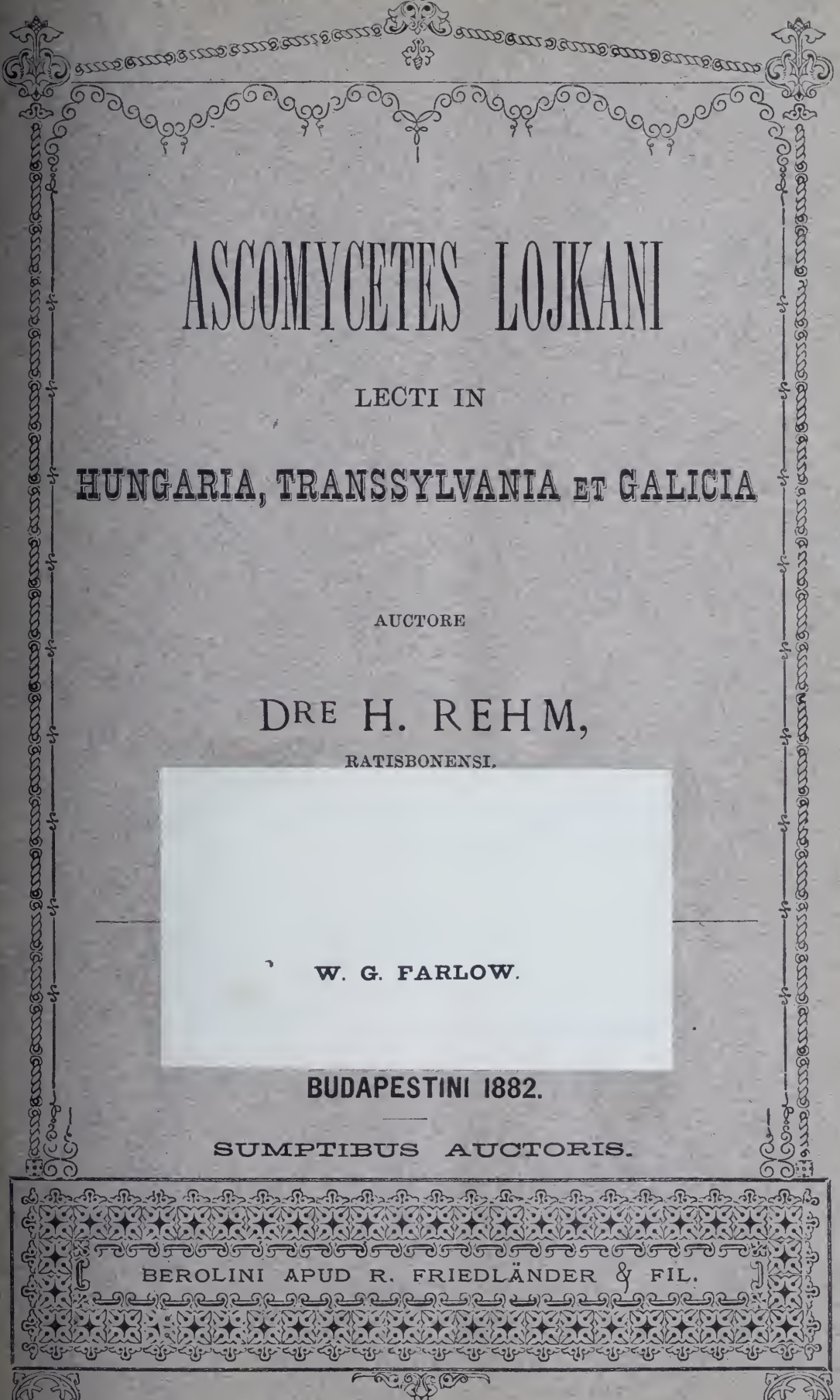




\section{$285 x=$}

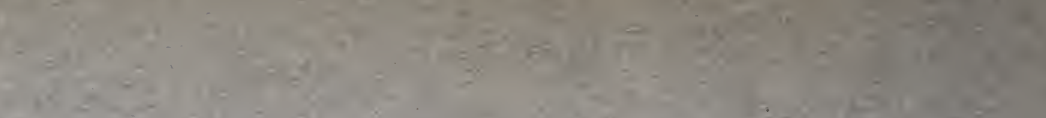

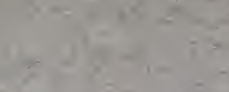

\&iv

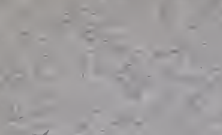

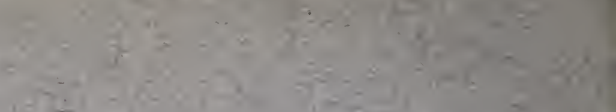

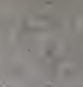

8

(axis

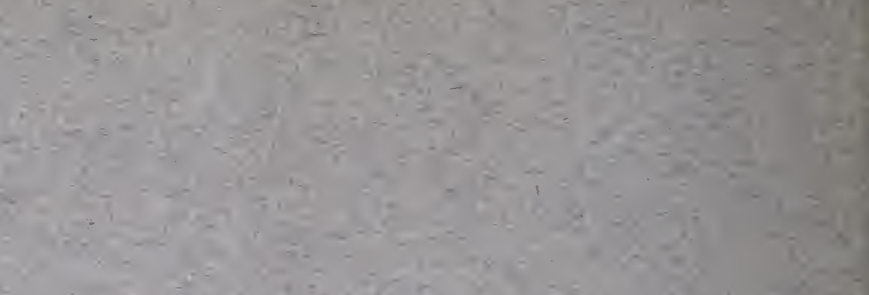

cest

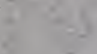

$-8, \frac{1}{3}$

F.

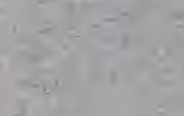

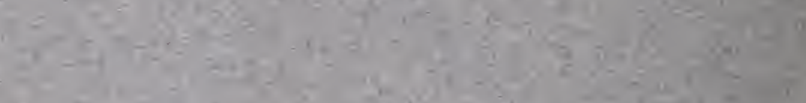

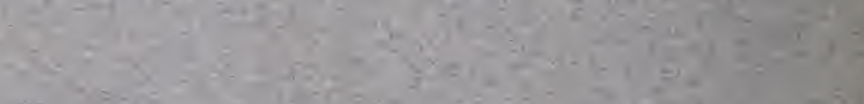

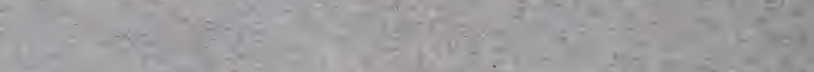

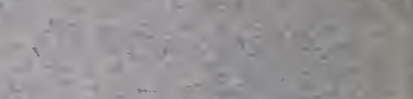

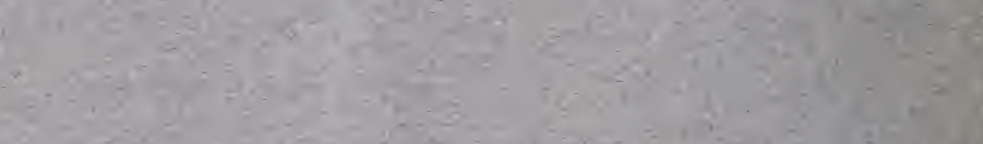

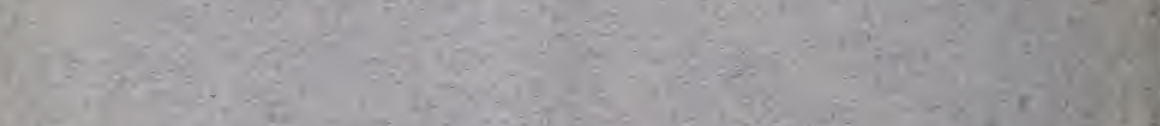

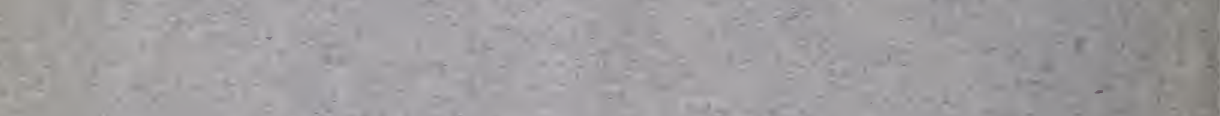

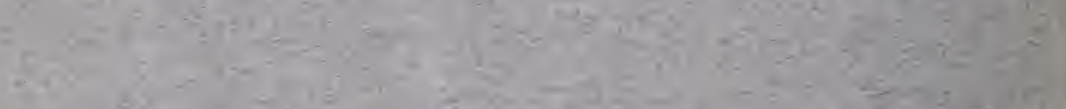

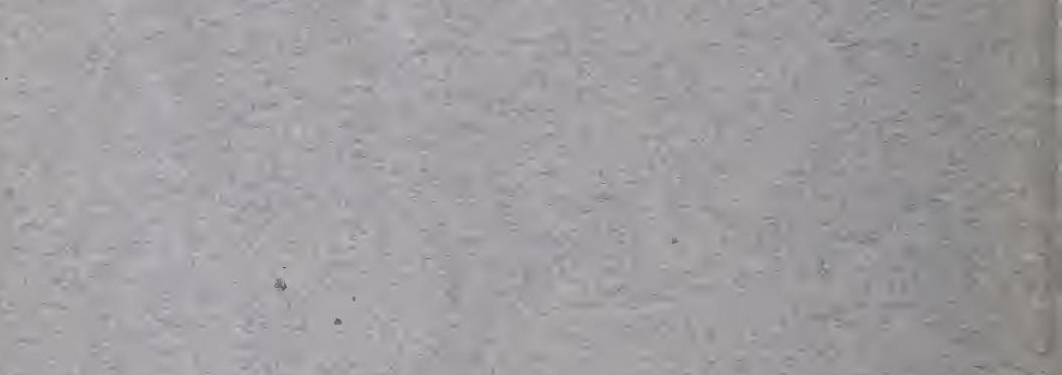

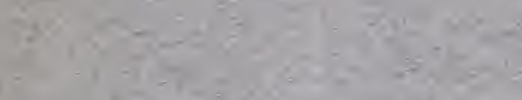

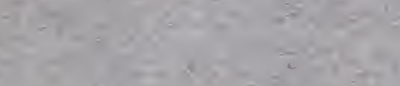

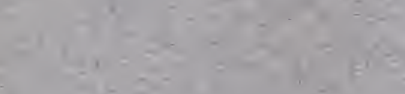
-

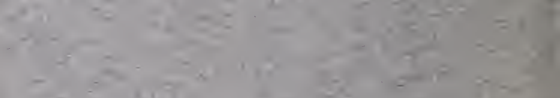

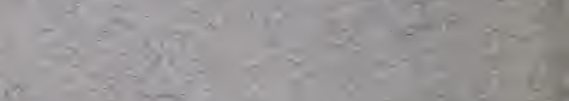

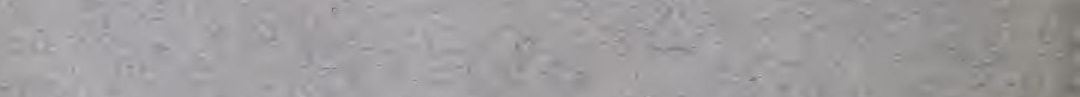

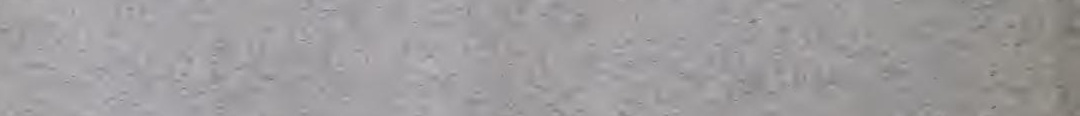
$-1$

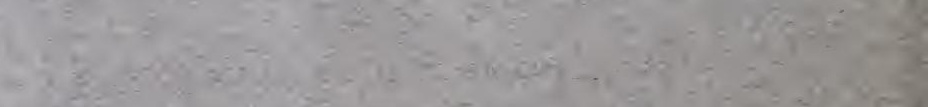

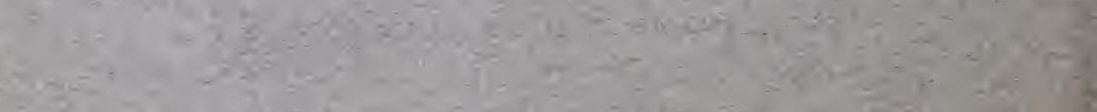

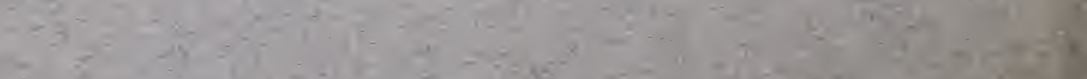

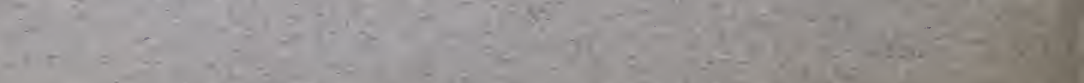




\section{ASCOUICLETES LOJKAVI}

LECTI IN

HUNGARIA, TRANSSYLVANIA ET GALICIA

AUCTORE

DRE ㅍ. REFAM,

RAXXSBONENSX.

BUDAPESTINI 1882.

- - S U UIPTIBUS AUOTORIB. $\rightarrow-\infty$

BEROLINI APUD R. FRIEDL ÄNDER \& FIL. 
EX OFFICINA JOSEPHI FANDA, Budapestini. 


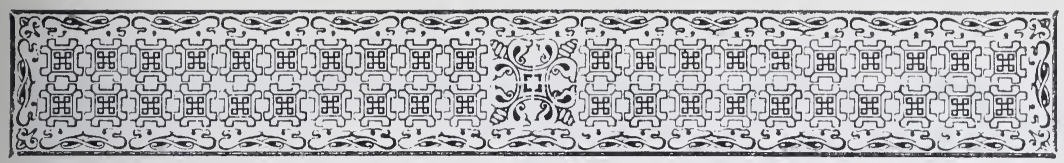

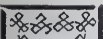

A cissimus et studio Lichenum praestantissimus, quoscunque Ascomycetes in itineribus per Hungariam et imprimis alpes Transsylvanas necnon per Galiciam factis, collegerat, eos mihi ad usum litterarum conferendos committeret. Sed demum longo post tempore fieri potuit, ut ex messe allata magnum Ascomycetum numerum his paginis mihi describendum esse constituerem, haud ignorans, perdifficile esse - id, quod cuivis Ascomycetum pescrutatori perspicuum est, - in exquirendis et comparandis illis speciebus accedere ad veritatem. Itaque, quantum in me situm erat, spectatione microscopii ope facta comparatisque inter se diligentissime speciebus et exsiccatis et descriptis, ad veritatem accedere conatus sum.

Specimina archetypa specierum omnium descriptarum in herbario meo reposita sunt; species autem plures in Ascomycetibus meis exsiccatis distributae in collectiones cryptogamicas insignes transierunt.

Jam descriptio specierum haud prorsus systematico ordine efficitur; multo plus operae collocavi in afferendis synonymis, litteris et collectionibus exsiccatis, quascunque usque ad hunc diem comparare et microscopii ope examinare mihi licuit. Ita quivis in posterum comparandi facultatem habebit, eo faciliorem, quod summam eorum, quae de Hymenii partibus egeram, accuratissime descriptam inveniet.

Magna copia Ascomycetum rariorum novorumque, ut dicunt, ex his regionibus prodiit magnam partem jam ab aliis auctoribus comprobatorum. Praeterea etiam illud apparet, multas species dis- 
tributas esse per alpes editissimas Helveticas, Tyrolenses usque ad Transsylvanas.

Itaque valde optandum est, ut et in posterum ad amoenas illas regiones, ditissimas, ut videtur, Ascomycetum quoque, perscrutator ille, usu peritissimus et indefessus, iterum iterumque redeat, copiam novam addens collectionibus Lichenum.

Me autem etiam posthac ex studio eorum summum gaudium percepturum et in iis examinandis diligentissime elaboraturum esse, ad usum litterarum, polliceor.

Castris Reginae (Regensburg) die 15 mensis novembris 1881. 


\section{Discomycetes.}

1. Humaria coprinaria (Cooke mycogr. p. 82 pl. 38 , f. 149 sub Peziza).

haud dubie varietas Humariae stercoreae (Pers.)

Sporidia hyalina, oblonga, obtusa, 1 cellularia, glabra, $15-18 / 8-9 ; 8$ monosticha in ascis cylindraceis, c. 12 mikr. lat. Paraphyses filiformes, apice sensim -6 micr. lat. clavatae ibique aurantiace granulatae. Pili perithecii fusci, septati, glabri, acutati, simplices. Jod -

Ad fimum vaccinum in Galicia australi 1869 leg. Lojka.

Ad fimum vaccinum prope Sumjácz. (Gömör) 1872 leg. Lojka sub 1866.

? Ad stercus human. Kaposvár (Somogy) Lojka 174.

Ad fimum vace. prope Eperjes 1869. Lojka.

Ad fimum vace. juxta introitum antri prope Ponor-Ohába (Hunyad) Transsylvaniae 1875. Lojka.

2. Humaria tenuis Fuckel symb. myc. p. $322 \mathrm{~s} \mathrm{e} \mathrm{c.} \mathrm{descriptionem.}$ c fr. Cooke mycogr. pl. 30 f. 116.

ex s. : Rehm Ascomyc. 404. (n o n Fuckel f. rhen. 2209 propter defectum pilorum perithecii).

Sporidia obtuse elliptica, hyalina, glabra, nucleo magno centrali instructa, 18-21/12-14; monosticha in ascis cylindraceis, $15-18$ mikr. lat. Paraphyses filiformes, hyalinae, sensim apice -5 mikr. crassae. Pili peritheciorum simplices, fusci, basi bulbosi, glabri, Jod -.

In monte Aksamitka prope Szczawnica in Galicia ad terram. Lojka sub 284.

3/a. Humaria theleboloides (Alb. et Schw. sub Peziza) e fr. Grevillea III f. 118. Cooke mycogr. I p. 83. pl. 39 f. 151. Michelia p. 430 . 
syn.:? Ascobolus granulatus Fuckel symb. myc. p. 287.

exs.: Rabh. f. eur. 621 (c. pilis). Phillips elv. brit. I 20. Cooke f. brit. I 571. Cooke f. brit. II 185. Fuckel f. rhen. 1876 (sub P. granulata). Rehm Ascomyc. 604.

Sporidia obtuse elliptica, glabra, hyalina, 1 cellularia, $15-18 / 6-8 ; 8$ monosticha in ascis cylindraceis $120 / 9$. Paraphyses filiformes, apice elliptice-clavatae, - 5 mikr. crassae. Jod -

Ad fimum vaccinum. Introitus antri prope Ponor-Ohálıa (Hunyad) Transsylvaniae. 1875 Lojka.

$3 / b$. Humaria theleboloides (Alb. et Schw. sub Peziza) va r. rubra (Cooke sub Peziza).

s yn: Peziza rubra Cooke mycogr. p. 83 pl. 39 f. 152.

exs: Cooke f. brit. I 572, II 186. Fuckel f. rhen. 2081 (sub Peziza stercorea).

Sporidia obtuse elliptica, glabra, hyalina, 1 cellularia, 14-18/9; 8 monosticha in ascis cylindraceis. Paraphyses apice clavatae, filiformes, hyalinae, clava elliptica -8 mikr. crassa. Perithecia haud pileis conspicuis instructa, ut apud Cooke 1. c., extus subgranulosa, crassa, in stercoris superficie longelateque aeruginose tincta, sessilia (quod idem in Fuckelii exs.). Jod -

Ad fimum vaccinum. Malomviz (Hunyad) Transsylvaniae. 1872. Lojka sub 1876, 1877.

4. Humaria umbrorum Fuckel symb. myc. p. 323.

s yn: Peziza umbrata Cooke hdb. p. 682. Peziza umbrosa Fr.

c f r. Cooke mycogr. I. p. 76 pl. 33 f. 138. Nyl. pez. fenn p. 122.

exs: Rabh. hb. myc. II 626 (sub P. uinbrosa). Fuckel f. rhen. 2080. Phillips elv. brit. 65 (sub P. umbrata). Rehm Ascomyc. 555. Sydow myc. march. 150.

Sporidia obtuse elliptica, hyalina, 1 cellularia, plerumque nucleo uno magno centrali praedita, episporio verruculoso, 18-20/12; 8 monosticha in ascis cylindraceis. Paraphyses filiformes, superne 6 mikr. crass. clavatae, fere hyalinae. Pili peritheciorum longi, simplices, septati, glabri, fusci, subacuti, basi c. 12 mikr. crassi. Jod -

Ad terram prope Kaposvár (Somogy) 1871. Lojka sub 199. 5. Humaria luteopallens (Nyl. pez. fenn. p. 18 sub Peziza). c f r Winter in Flora 1872 p. 509 . Grevillea III f. 72 tab. 31 f. 72. Cooke mycogr. I. p. 85 pl. 40 f. 156. exs: Rehm Ascomyc. 7 sub Pyronema. 
Sporidia obtuse - elliptica, glabra, hyalina, 1 cellularia, $12-17 / 8-9,8$ monosticha in ascis cylindraceis. Paraphyses filiformes, hyalinae, apice vix clavatae. Pili marginales fusciduli, obtusi, septati, glabri. Jod -.

Ad viam arenosam horti pastoris ecclesiae. Gelsendorf (Stryj) in Galicia australi 8/1869. Lojka.

6. Pustularia cerea (Sow. sub Peziza) Rehm. (potius fortasse var. Pustulariae vesiculosae (Bull sub Peziza).

syn:? Plicaria - Fuckel symb. myc. p. 327. (sporarum descriptio no $n$ quadrat).

e f r : Nyl. pez. fenn. p. 13. Cooke hdb. p. 671, myengr. I pl. 64 f. 244 . Karsten myc. fenn. II p. 54 . Michelia VI p. 164.

exs: Fuckel f. rhen. 1225. Ellis f. n. am. 135. Rehm Ascomyc. 201.

Sporidia oblonga, obtusa, glabra, unicellularia, hyalina, $15-18 / 9-10 ; 8$ monosticha in ascis cylindraceis, usque 18 mikr. crassis. Paraphyses filiformes, apice sensim usque 7 mikr. crass. clavatae, hyalinae.

Jodii ope apex ascorum valde coerulescit.

Ad terram prope Kaposvár (Somogy) 1872 Lojka sub 145.

7. Pustularia repanda (Wahlbg sub Peziza)

syll: Peziza Stephensoniana Ellis in herb. meo, quod explr convenit cum explre Phillipsii in herb. meo sub Peziza repanda Whlbg.

c fr. Nyl. pez. fenn. p. 14. Karsten myc. fenn. II p. 54.

I o n conveniunt: Cooke hdb. p. 670 (sporidia rough!), Fuckel symb. myc. p. 328 (episporium granuloso-tuberculosum fuscum), Cooke mycogr. p. 142 pl. 62 f 240 (sporidia elliptica, binucleata, $18-20,10)$.

Sporidia obtusa, oblonga, hyalina, glabra, unicellullaria, enucleata, 12-14/8; monosticha in ascis cylindraceis, -12 mikr. crassis ; Paraphyses paucae, hyalınae, filiformes, apice sensim 3 mikr. crass.

Jodii ope asci valde coerulescunt.

(Quum incertum apparet, quid sit vera Peziza repanda Wahlenbergii, potius pro hac specie nomen supra allatum Ellisii sumendum erit.)

Ad terram prope Kaposvár (Somogy). 1872 Lojka sub 74.

Ad terram prope Javorina (Szepes) 1869 Lojka sub 638.

8. Pustularia Tarzetta (Cooke sub Peziza nov. spec in litt. ad me d. d. 18/III 1874) 
c fr. Winter in Flora 1872 p. 425. Cooke in Grevillea II p. 176 ; mycogr. I p. 166 pl. 74 f. 287.

exs.: Rehm Ascomycetes 53 (sub Peziza vesiculosa).

Descriptio cl. Cookei l. c.: „Stipitata vel subsessilis. Cupula cyathiformis, regularis, pallens, subfarinosa, deorsum in stipitem gracile attenuata; margine leniter incurvo, laevi. Hymenio rubescente. Ascis cylindraceis Sporidiis ellipticis, laevibus 15/7. Paraphysibus sursum incrassatis."

Jodii ope asci non coerulescunt.

Ad terram prope Kaposvár (Somogy). Lojka 5/1871 sub 118.

9. Plectania coccinea (Jacq. sub Peziza) Fuckel symb. myc. p. 324 .

c f r. Karsten myc: fenn. II p. 43. Grevillea III f. 87 Cooke mycogr. I p. 55 pl. 25 f. 95. Nyl. pez. fenn. p. 11.

exs: Fuckel f. rhen. 1213. Cooke f. brit. I 651. Phillips elv. brit. 61. Saccardo myc. Ven. 1512.

Sporidia oblongo-elliptica, hyalina, glabra, 1 cellularia, nucleo 1 parvulo praedita, $24-30 /-10 ; 8$ monosticha in ascis cylindraceis, c. 10 mikr. crass. Paraphyses filiformes, haud clavatae, granulis rubris imbutae. Jod -.

Ad ramulum siccum. Kaposvár (Somogy) 1872 Lojka sub 31.

10. Pyronema omphalodes (Bull. sub Peziza) Fuckel symb. myc. p. 319.

s y n: Pyronema Marianum Carus, P. confluens Tul., ? P. Franzonianum De N.

c fr. Cooke hdb. p. 676. mycogr. I p. 37 tab. 17 f. 65. Grevillea III f. 50. Karsten myc. fenn. II p. 48. Saccardo f. Ven. nov. IV p. 38. Michelia VII p. 328.

exs: Fuckel f. rhen. 1218. Rabh. f. eur. 708 (? 267).

Sporidia oblongo-elliptica, obtusa, hyalina, glabra, 1 cellularia, $12 / 7 ; 8$ monosticha in ascis cylindraceis. Paraphyses articulatae (?), haud perspicue clavatae, c. 3 mikr. crassae. Jod -.

Ad terram limosam prope Soborsin. 7/1872 Lojka sub 1871.

12. Helotium citrinum (Hedw. sub Octospora) Fr. S. Veg.

syn: Peziza - Batsch. Helotium lenticulare Fuckel symb. myc. p. 315 .

c fr. Nyl. pez. fenn. p. 43. Karsten myc. fenn. II p. 120. Saccardo myc. Ven. 165. Cooke hdb. p. 712 (? Fuckel symb. myc. 1. 315 .)

ex s: Mougeot et Nestl. stirp. Vog. 784. Rabh. f. eur. 29 a, 
b. Schweiz. crypt. 118. Fuckel f. rhen. 1151 (sub H. lenticulare), n 0 n Tuckel f. rhen. 1152 (explr mancum in herb. meo).

Sporidia oblonga, obtusa, hyalina, 1 cellularia, duobus nucleis magnis instructa, $10-12 / 4 ; 8$ monosticha, superne saepe disticha in ascis clavatis. Paraphyses filiformes, apice haud clavatae, c. 1,5 mikr. crassae. Perithecium prosenchymaticum, flavidulum. Jodii ope apex ascorum coerulescit.

Ad ramulum fagineum decorticatum vallis "Raj" prope SzepesOlaszi. 1869 Lojka sub no 637.

12. Helotium cyathoideum (Bull. sub Peziza) Karsten myc. fenn. I p. 136.

syn: Octospora albidula Hedw. Peziza - Wahlbg. Helotium cyathoideuin var. - Karsten myc. fenn. I p. 137. Cyathicula vulgaris DN. (? Peziza Cacaliae Pers., Solani Pers.)

cfr. Cooke hdb. p. 699. (? Fuckel symb. myc. p. 307.)

exs: Cooke f. brit. I 594, II 377, 378. Thümen f. austr. 1115. myc. un. 416. Phillips elv. brit. 79. Fuckel f. rhen. 1179. Kunze f. sel. 294 (sub P. Cacaliae), 183 (sub P. Solani) (? 381). Rehm Ascomyc. 306. Rabh. f. eur. 2207 (sub P. Solani) (? 224, 2408).

Sporidia oblongo-elliptica, subacuta, recta, hyalina, 1 cellularia, saepe nucleis duobus apicalibus praedita, $6-10,1,5 ; 8$ disticha in ascis clavatis $-45 /-5$; paraphyses filiformes, apice vix (-2 mikr.) crassiores. Perithecium prosenchymaticum, tlavidulum. Jodii ope apex ascorum coerulescit.

a. ad caules siccas Dipsacisylv. prope Lunkány in Hung. 1872 Lojka.

b. ad caulem putrescentem Aconiti in alpe Dzurowa (Liptó) c. $5000^{\prime} 1872$ Lojka.

c. ad caules siccas Heraclei palmati infra alpem Retyezát (Hunyad) Transsylvaniae c. 6000'. 1873 Lojka.

d. ad caules siccas Artemisiae vulg. in horto Esterházyano, Kaposvár (Somogy). 1871 Lojka.

(differt peritheciis crassioribus, fuscidulis).

13. Helotium cyathoideum (Bull. sub Peziza) Karsten mye. fenn. I p. 136. f. dolos ellum Karsten myc. fenn. I p. 138.

Sporidia 9-12/2; Asci 45-50/5-6.

Ad caules siccas Angelicae prope lacum Zenoga infra alpem Retyezát (Hunyad) Transsylvaniae. 1873 Lojka.

14. Holotium glanduliforme Rehm nov. spec. 1875 .

Perithecia sparsa, cyathoidea, crasse stipitata, stipite fuscidulo, 
basi subhyalino, turbinata, c. $1-1,5 \mathrm{~mm}$ alta, $0,5-7 \mathrm{~mm}$ diam., sicca margine urceolato, involuto, fusca, humida concavo-urceolata, flavidula, prosenchymatice flavide contexta, glabra, sicca extus subruguloso-striata. Asci clavati, 8 spori, 45/6; Sporidia elliptica, subobtusa, hyalina, recta, 1 cellularia, disticha, 9/2-2,5. Paraphyses filiformes, superne sensim - 2,5 vel 3 milkr. crassae, ascos superantes. Jodii ope apex ascorum coerulescit.

exs: Rehm Ascomyc. 255, 559.

syn. ? Helotium cyathoideum (Bull). f. multicolor Karsten myc. fenn. p. 137.

Ad ramulos siccos Ononidis spinosae. Szent-Miklós in insula Danubii Csepel (Pest). Lojka sub 2001.

15. Helotium herbarum (Pers. sub. Peziza) Fr. S. V. Sc.

ef r Fuckel symb. myc. p. 316. Nyl. pez. fenn. p. 45. Cooke hdb. p. 714. Karsten myc. fenu. I p. 117.

ex s: Moug. et Nestler st. Vog. 785. Rabh. hb. myc. II 227. f. eur. 218 (sub P. rubi v. herbicola), 810. Fuckel f. rhen. 1147. Cooke f. brit. II 391. Phillips elr. brit. 87. Kunze f. sel. 187, 572. Rehm Ascomyc. 12. Saccardo myc. Ven. 1506 (sub H. scutula f. Urticae); ? Fuckel f. rhen. 1200 (sub P. caulicola). ? Ellis n. am. f. 670.

f. h e r bicola Karsten myc. fenn. I p. 118.

Sporidia fusoideo-elongata, subacuta, plerumque curvula, hyalina, $1-2$ cellularia, 4 nucleolis praedita, $9-12,1,5 ; 8$ disticha in ascis clavatis $-65 / 6$. Paraphyses filiformes, hyalinae. Jodii ope apex ascorum coerulescit.

Ad caules putrescentes Umbelliferarum prope Kaposvár (Somogy) 1871 sub 204 et 208 Lojka.

16. Helotium Humuli (Lasch sub Peziza) DN. in ann. critt. I p. 379.

s y n: Peziza humilis Desm.

cf r Fuckel symb. myc. p. 308. Karsten myc. fenn. I p. 112. Saccardo myc. Ven. p. 211.

ex s : Rabh. f. eur. 1185 (Jod -), 1421, hb. myc. II 221. Saccardo myc. Ven. 960 (Jod -). Rehm Ascomyc. 60. Sydow myc. march. 269.

Sporidia elongato-elliptica, plerumque acutata, recta vel subcurvata, hyalina, 1 cellularia, nucleis $2-4$ magnis instructa; $15-18 / 4 ; 8$ disticha in ascis clavatis $-80 / 9$. Paraphyses filiformes, superne $-2,5$ mikr. incrassatae. Jodii ope apex ascorum coerulescit.

Ad sarmenta sicca Humuli Lupuli in vinetis prope Kaposvár (Somogy) 1870 Lojka in Rehm Ascomyc. 
17. Helotium Urticae (Pers. sub Peziza) Karsten myc. fenn. I p. 133.

syn: Peziza striata Fr. P. Cacaliae f. Senecionis Fuckel symb. myc. p. 307. P. Cacaliae Sacc. in Michelia I p. 42.

ef r Nyl. pez. fenn. p. 39. Fuckel symb. myc. p. 307.

exs: Thümen f. austr. 1116 (sub Cyathicula De N.), myc. un. 1811 (sub H. Cacaliae var. Amaranthi Sacc.). Rehm Ascomyc. 59. (? Fuckel f. rhen. 1180, 2283 sub P. Cacaliae f. Senecionis; Rabh. f. eur. 221).

Sporidia elongato-fusiformia subobtusa, plerumque recta, hyalina, 1 cellularia, $8-9 / 2 ; 8$ disticha in ascis clavatis, 50/6 ; Paraphyses filiformes. Jodii ope apex ascorum coerulescit.

a. Ad caules putridas Umbelliferarum in horto Esterházyano Kaposvár (Somogy) 1871 Lojka in Rehm Ascomyc.

b. Ibidem ad caules putr. Lapparum. 1871 Lojka.

c. Ibidem ad caulem Daturae Stramonii 1871 Lojka sub $117 \mathrm{c}$.

d. Ad caules putridas Polygoni Persicariae prope Malomviz in Transsylv. 1872. Lojka.

18. Helotium virgultorum (Vahl sub Peziza) Fr. syst.

e f r Fuckel symb. myc. p. 314. Nyl. pez. fenn. p. 37, 38. Karsten myc. fenn. I p. 109.

f. fructigenu $u$ (Bull. sub Peziza).

cfr Karsten myc. fenn. I p. 113 (? Cooke hdb. p. 709, sporae acicular-oblong!)

exs: Fuckel f. rhen. 1154. Rabh. hb. myc. II 510 (? 121), Thiumen f. austr. 929. Cooke f. brit. I 479, II 392. Kunze f. sel. 386. Rehm Ascomyc. 9, 10.

Sporidia elongato-fusiformia, recta vel subcurvata, 1 cellularia, plerumque nucleis 2 magnis praedita, hyalina, $15-18 / 4,5-5 ; 8$ disticha in ascis clavatis, tenuibus, apice subincrassatis, 100,-12; Paraphyses filiformes, apice sensim - 3 mikr. crassae. Jodii ope apex ascorum coerulescit.

Ad dejectos fructus quercinos. Gelsendorf (Galicia australis) 1869 Lojka.

19. Helotium virgultorum (Vahl sub Peziza) Fr.

v a r. s cutula (Pers. sub Peziza) Karsten mye. fenn. I p. 110. c f r ? Fuckel symb. myc. p. 308.

ex s: Fuckel f. rhen. 2477. Rabh. f. eur. 2105. Rehm Ascomyc. 56 (? Saccardo myc. Ven. 728. Phillips elv. brit. 120a). 
Sporidia elliptica, plerumque una apice obtusata, recta vel subcurvata, 1 cellularia, plerumque nucleis 2 magnis praedita, hyalina, 18-21/4-5; 8 disticha in ascis clavatis, 90/8. Paraphyses filiformes, hyalinae. Jodii ope apex ascorum coerulescit.

Ad caules putridas Artemisiae vulg. in horto Esterházyano. Kaposvár (Somogy) 1871 Lojka in Rehm Ascomycet.

21. Leucoloma convexellum (Karsten myc. fenn. I p. 64 sub Peziza) Rehm.

efr Cooke mycogr. I p. 22 pl. 9 f. 35.

Sporidia globosa, hyalina, glabra, 12-14 mikr. diam. 8 monosticha in ascis cylindraceis c. 16 mikr. crass. Paraphyses filiformes, apice curvatae et sensim 3 mikr. crassae. Jod -.

In excavatione terrae vallis "Riu mare" infra alpem Retyezát Transsylvaniae. 8/1872 Lojka sub 1870.

21. Leucoloma tetrasporum Fuckel symb. myc. p. 317.

sy n: Ascobolus-Fuckel in IJedwigia 1866 p. 4 t. 5 f. a-c.; Peziza-Cooke in Grevillea III f. 82.

cfr ? Cooke mycogr. I p. 27 pl. 12 f. 45. (delineatio sporarum et paraphysum non quadrat, sporae $-35 / 10$ ).

exs: ? Fuckel f. rhen. 1856 (nimis mancum explr herb. mei).

Sporidia elliptica, obtusa, glabra, hyalina, 1 cellularia, nucleo magno 1 (rarius 2) centrali instructa, 18-20/10; 4 monosticha in ascis cylindraceis c. 105/12; Paraphyses filiformes, guttulis rubescentibus repletae, apice $7-8$ mikr. crassa clava praeditae. Jor -

Ad terram. Kaposvár (Somogy). 10/1871 Lojka sub 211.

(Species valde dubia, nempe in sporidiorum saepe abortivorum numero condita.)

22. Dasyscypha calycina (Schum. sub Peziza) Fuckel symb. myc. p. 305 .

syn: Helotium-Karsten myc. fenn. I p. 154. Nyl. pez. fenn. p. 24.

Sporidia obtusa, elliptica, glabra, 1 cellularia, hyalina, 9/5; $81-1^{1} / 2$ sticha in ascis clavatis 90/7--9. Paraphyses filiformes, guttulatae, apice vix crassiores.

Ad ramulos Pini Pumilionis; Válye-Rásza infra montem Retyezát Transsylvaniae. 8/1872 Lojka.

Exemplaria manca, itaque dubie ad hanc speciem allata.

23. Dasyscypha chrysophthalma (Pers. sub Peziza) Rehm.

s y n: Helotium-Karsten myc. fenn. I p. 155. Peziza calycina $\beta$ Abietis Fr. Syst. Peziza pulchella $\beta$ Havococcinea Alb. et Schw 
Pithya suecica Fuckel symb. mye. Nachtrag III p. 32. (m in i me = P. chrysophthalma Gerard, efr Hedwigia 1874 p. 29).

ef r Nyl. pez. fenn. p. 34. Grevillea III p. 127 tab. $40 \mathrm{f}$. 168, IV p. 41.

exs: Rehm Ascomyc. 108 (sub Pithya). Thümen f. austr. 1213. Fuckel f. rhen. 2686 (sub P. suecica).

Sporidia globosa, hyalina, glabra, 1 cellularia, $4-5$ mikr. crassa, 8 monosticha in ascorum cylindraceorum parte superiore, c. 60/6. Paraphyses filiformes. Jod -

Ad ramulos Pini Pumilionis. Tatra "Hintere Leithen" c. 5500. 9/1869. leg. Lojka sub 277.

24. Dasyscypha coerulescens Rehm nov. spec. 1873.

Perithecia gregaria, sessilia, primitus globosa, dein urceolata, denique applanata, margine spurio circumdata, coerulescentia, c. 0,5-6 $\mathrm{mm}$ diam. prosenchymatice contexta, pilis brevibus, scabris, obtusis, simplicibus, 4-6 mikr. crassis obsessa. Asci clavati, 24-30/3-4, 8 spori. Sporidia oblongata, obtusa, 1 cellularia, hyalina, $4-5 / 1$, disticha. Paraphyses simplices, filiformes, tenerae, haud clavatae Jodii ope apex ascorum dilute coerulescit.

Ad corticem Betulae prope Chodowice (Stryj) Galiciae australis. Lojka sub 41 .

25. Dasyscypha lanata Rehm nov. spec. 1879.

Perithecia aperta, explanata, sessilia, solitaria, sicea corrugata, humida disco flavo plano, extus hirta, alba, parenchymatice flavide contexta, pilis septatis, hyalinis, glabris, simplicibus, obtusis, longis, c. 4 mikr. crass. obsessa, $-1,2-5 \mathrm{~mm}$ diam. Asci clavati, 8 spori, 60/4. Sporidia bacilliformia, obtusa, recta vel subcurvata, 1-2 cellularia, 9-15/1-1,5, hyalina, disticha. Paraphyses filiformes, tenerae, apice, subincrassatae. Jodii ope apex ascorum coerulescit.

Ad caules putrescentes Aconiti Napelli infra alpem Retyezát (Hunyad) Transsylvaniae. Lojka.

Species videtur propria, ad Dasysc. leucophaeam vergens.

26. Dasyscypha leucophaea (Pers. sub Peziza) Rehm.

syn: Lachnum-Karsten myc. fenn. I p. 177. Trichopeziza sulphurea Fuckel symb. myc. p. 296. (? Peziza sulphurea Fr. syst. myc. II p. 104.)

c fr Cooke hảb. p. 689. Nyl. pez. fenn. p. 31. Saccardo f. Ven. IV p. 34. Michelia VI p. 79. [sec. Saccardo differt a Tr. sulphurea (Fr.) Fuckel.]

exs: Rabh. hb. myc. II 707 (sub P. sulphurea); Fuckel f. 
rhen. 1196. Cooke f. brit. II 372. Phillips elv. brit. (? 25) 166. Saccardo myc. Ven. 1518 (sub Tr. sulphurea). Rehm Ascomye. 65 $a, b$. (? Thümen myc. un. 1812). Perithecia prosenchymatica, flavidula, pilis flavis, septatis, scabris, c. 4,5 mikr. crassis obsessa; Asci cylindraceo-clavati c. $60 / 5,8$ spori. Sporidia recta vel subcurvata, elongato-elliptica, subobtusa, 1 cellularia, hyalina, 9-14/1, 5-2 disticha. Paraphyses lanceolato-acuminatae, ascos superantes, c. 45 mikr. crass. Jodii ope apex ascorum cocrulescit.

a. Ad caules putridas Umbelliferarum in lıorto Esterházyano prope Kaposvár (Somogy) Lojka 1871. (edita in Rehm Ascomyc. $65 a !)$

b. Ad caules putridas Conii maculati. Malomviz (Hunyad) Transsylvaniae 8/1873. Lojka.

\section{Dasyscypha leucostoma Rehin.}

Perithecia gregaria, sessilia, globosa, urceolata, extus fuscobrunnea vel fusconigra, hirta, ore connivente albo ex fibris hyalinis constituto, humidata aperta, disco dein conspicuo concavo pallido, c. $0,4 \mathrm{~mm}$ diam., prosenchymatice fusce contexta, pilis longis, fuscidulis, obtusis, subscabris, simplicibus, c. 3 mikr. crassis obsessa. Asci cylindraceo-clavati, apice incrassati $60-90 / 5,8$ spori. Sporidia oblonga vel subfusiformia, plerumque recta, 1 cellularia, hyalina, 9-16 1,5-2, disticha. Paraphyses filiformes, apice haud clavatae. Jodii ope apex ascorum coerulescit.

exs: Rehm Ascomyc. $205 a, b$ (sub Tapesia); Thümen myc. uil. 610 .

( Ad caules putridas Aconiti Napelli aliarumque herbarum circa lacum Zenoga infra montem Retyezát (Hunyad) Transsylvaniae, c. $6000^{\prime}$ alt. 8,1873 Lojka (edita in Rehm Ascomyc. 205 a). Lo cu s primarius!

b. ibidem in alpe Dzurowa (Liptó) alt. c. 5000! 1872 Lojka.

Species distinctissima et ab illo tempore in Alpibus editissimis Tyrolensibus et Helveticis saepe reperta.

28. Dasyscypha mollissima (Lasch sub Peziza) Rehm.

syn: Lachnum - Karsten myc. fenn. I p. 147.

cf r: Nyl. pez. fenn. p. 31.

exs: Rabh. hb. myc. II 708. Kunze f sel. 567.

Exemplar dubium, quoad Hymenium plane inevolutum et immaturum.

Ad caules putridas Conii maculati in horto Esterházyano Ka"posvár (Somogy) 5/1871 Lojka. 
29. Dasyscypha rolicina (Fr. sub Peziza).

s y n: Trichopeziza - Fuckel symb. myc. 1. 296. Lachnum Karsten myc. fenn. I p. 182. Peziza Atropae Pers. sec. Rabh. D. crypt. Flora I p. 358. Excipula phaeotricha Rabh.

e f r. Michelia VI p. 164 (sporidia $8-10 / 1-1,5)$.

ex s: Fuckel f. rhen. 1197. Moug. et Nestler stirp. Vog. 686 (sub P. Atropae). Rabh. hb. myc. II 515. Rehm Ascomyc. 204.

Perithecia prosenchymatica, fuscidula, pilis septatis, glabris, apice obtusis, fuscis, c. 6 mikr. crassis, longis obsessa. Asci clavati, apice incrassati, 8 spori, $60-70 / 6-8$. Sporidia oblonga, obtusa, recta vel subcurvata, hyalina, 1 cellularia, 12-15/2, 5-3, disticha. Paraphyses lanceolatae, apice acuminatae, c. 2,5 mikr. crassae, ascos superantes. Jodii ope apex ascorum coerulescit.

Ad caules putridas Aconiti circa lacum Zenoga infra montem Retyezát (Hunyad) Transsylvaniae alt. e. 6000'. 8/1873 Lojka.

Edita in Rehm Ascomyc. 204.

30. Dasyscypha resinaria (Cooke et Phillips sub Peziza).

c fr. Grevillea III p. 185. Winter in Flora 1872 p. 495.

exs: Phillips elv. brit. 66. Rehm Ascomyc. 55 (sub Pithya chrysophthalma).

Perithecia prosenchymatica, hyalina, pilis hyalinis, scabris, simplicibus, c. 4 mikr. crassis obsessa; Asci clavati, 30-36/4, 8 spori. Sporidia subrotunda, 1 cellularia, hyalina, 2,52, disticha. Paraphyses filiformes, c. 1,5 mikr. crassae. Jodii ope apex ascorum coerulescit.

Ad corticem abietis in regione "Male Brunowo" infra alpem Kralowa Hola prope Teplicska (Liptó) 8/1872 Lojka.

31. Dasyscypha villosa (Pers. syn. f. p. 655 sub Peziza).

s yn: Trichopeziza - Fuckel symb. myc. p. 296. Cyphella Karsten myc. fenn. I p. 175, III p. 325. Peziza syringea Wallr. Trichopeziza nivea Fuckel symb. myc. p. 296. (? Cyphella pezizoides Zopf in myc. march. no 1 sec. icon.).

c f r Nyl pez. feun. p. 32. Saccardo myc. Ven. p. 163 Cooke hdb. p. 690.

exs: Rabh. f. eur. $616 a, b$ (snb P. nivea), Fuckel f. rhen. 1199 (sub P. nivea); Rabh. hb. myc. II 225, 705 (sub nivea', f. eur. 32 (sub syringea), Thümen myc. ur. 1608 (n o n Fuckel f. rhen. 1193 (sub P. Aspidii), quam Fuckel 1. all. citat, nec f. rhen. 2286.)

Perithecia prosenchymatica, hyaline contexta, pilis longis, obtusis, scabris, hyalinis, septatis, c. 5 mikr. crassis obsessa. Hyme- 
nium sine ascis et sporidiis. Vix inter Ascomycetes collocanda species!

a. ad caules siccas Umbelliferarum prope Soborsin in Hungaria $7 / 1872$ Lojka.

b. ad sarmenta vini et ramulos sambucinos putrescentes. Kaposvár (Somogy) 5/1871 Lojka.

c. ad caules putrescentes Ononidis spinosac. Szent-Miklós in insula Danubii Csepel (Pest). 1873 Lojka sub 2001.

32. Tapesia fusca (Pers. sub Peziza) Fuckel symb. myc. p. 302.

s y n: Mollisia - Karsten myc. fenn. I p. 207 (? Niptera livido-fusca Fr. sub Peziza -, efr Nyl. pez. fenn. p. 61). Fucke symb. myc. Nachtrag II p 58.)

exs: Rabh. hb. myc. II 306. Fuckel f. rhen. 1173 (sub P. vulgaris), 1595, 2573 (sub Niptera lividofusca). Rehm Ascomyc. 153 (? Phillips elv. brit. 77, Cooke f. brit. II 556,654).

Asci clavati, apice incrassati, $-60 / 7-8,8$ spori, Sporidia oblongata, obtusa, 1 cellularia, hyalina, $9-11 / 3$, disticha. Paraphyses filiformes, superne sensim -3 mikr. crassae. Jodii ope apex ascorum coerulescit.

Ad ramum putridum Pini Cembrae prope lacum Zenoga (Hunyad) in Transsylv. Lojka 1873 sub 2412 \#.

33. Tapesia byssina Fuckel symb. myc. p. 302.

s y n ? Peziza melaleuca Fr. f. byssina.

ex s: Rehm Ascomyc. 153 b (sub Tapesia fusca).

Asci clavati, 50/6, 8 spori. Sporidia oblonga vel ovoidea, obtusa, 1 cellularia, hyalina, $9-11 / 3,11 / 2$ sticha. Paraphyses hyalinie, filiformes. Jodii ope apex ascorum coerulescit.

Ad lignum putridum prope Kaposvár (Somogy) 1872. Lojka sub 167.

Forsitan forma bysso deficiente diversa a: Niptera melaleuca (Fr. sub Peziza) Fuckel symb. myc. p. 292. syn: Patellaria - Fr. S. Veg. c fr Nyl. pez. fenn. p. 60 adn. Saccardo f. Ven. nov. IV p. 31. ? Michelia VI p. 81. e x s : Fuckel f. rhen. 2284.

34. Pezizella Aconiti Rehm nov. spec. 6,9 1873.

Perithecia gregaria, sessilia, orbicularia, primitus subglobosa, urceolata, dein plus minusve expansa, sicca albido-rosea, extus scabra; disco roseo; humida dilatata, margine crasso, albido, denticulato cincta, roseo-cinerea, disco plano, prosenchymatice tenerrime, subhyaline contexta, c. $3-5 \mathrm{~mm}$ diam Asci clavati, apice incrassati, 
8 spori, 45-50/7; Sporidia subelliptica, obtusa, 1 cellularia, 2 plerumque 4 nucleata, recta vel subcurvata, hyalina, disticha, 12 - 14/2,5-3. Paraphyses filiformes, tenerae, hyalinae. Jodii ope apex ascorum coerulescit.

ex s: Rehin Ascomyc. 163.

Ad caules putridas Aconiti Napelli in alpe Dzurowa prope Teplicska (Liptó) alt. c. 5000' Lojka 81873 sub 1378.

Edita in Rehm Ascomyc. 163.

35. Pezizella Hungarica Rehm nov. spec.

cf r. Winter in Flora 1872 p. 526.

Perithecia minutissima, c. $0,3 \mathrm{~mm}$ diam., gregaria, sessilia, primitus globosa, dein urceolata, sicca flavidula, humectata hyalinella, prosenchymatice contexta. Asci clavati, 8 spori, 30/4. Sporidia elongato-cuneiformia, recta, 1 cellularia, hyalina, obtusa, $5-7 / 1,5$, disticha. Paraphyses tenerae, filiformes. Jodii ope apex ascorum coerulescit.

ex s. Rehm Ascomyc. 64.

a. Ad caules putridas Umbelliferarum in horto Esterhízyano Kaposvár (Somogy) 1871 Lojka.

Edita in Rehm Ascomyc. 64.

b. Ad caulem putridam Heraclei palmati infra montem Retyezát (Hunyad) Transsylvaniae alt. c. 6000' 1873 Lojka.

(differt sporidiis p. p. subcurvatis, 6-8/1, paraphysibus apice globosa clava c. 3 mikr. crassa instructis).

36. Pezizella Tyrolensis Rehm nov. spec.

Perithecia plerumque sparsa, rarius gregaria, sessilia, primitus globosula, dein substipitata, serius explanata, sicca juniora aureovel aurantiaco-flavida, scabra, concava, plicata, margine subfimbriato et rugoso, dein explanata, disciformia, haud distincte marginata, margine albido-fiunbriata, humida flavida, expansa, prosenchymatice flavidule contexta, - 1 (vel 1,2) $\mathrm{mm}$ diam. Asci clavati, apice incrassati, 8 spori, $40-60 / 6-8$. Sporidia oblonga vel obtuse oblongata, elliptica, hyalina, recta, 1 cellularia, saepe 1 -2 nucleolis praedita, $6-9 / 2,5$; Paraphyses filiformes, apice haud clavatae, hyalinae, p. p. crassae ascosque superantes, c 2 mikr. diam. Jodii ope apex ascorum coerulescit.

exs: Rehm Ascomyc. 116. Thümen myc. un. 516.

Ad caules putridas Angelicae circa lacum Zenoga infra alpem Retyezát (Hunyad) Transsylvaniae 1873 Lojka.

(Forsitan et Pezizella Hungarica Rehm huc pertinet). 
Species distinctissima et per omnes alpes editiories juxta glaciem aeternam distributa.

37. Niptera cinerea (Batsch sub Peziza) Fuckel symb. myc. p. 292.

s y n: Peziza callosa Bull. P. salicaria Pers. Trochila - De N. Mollisia cinerea Karsten myc. fenn. I p. 189.

c f r. Nyl. pez. fenn. p. $60,61^{* *}$. Cooke hdb. p. 701. Saccardo myc. Ven. p. 16\%. Nichelia I p. 424.

exs: Rehm Ascomyc. 66 (sub Pyrenopeziza Artemisiae). (n o n = Saccardo myc. Ven. p. 371). Mollisia complicata Karsten myc. fenn. I p. 192 ut videtur).

Perithecia parenchymatice fusca. Asci clavati, $45-50,5 ; 8$ spori. Sporidia obtusa, elongato-elliptica recta vel subcurvata, 1 cellularia, utraque apice nucleo parvulo instructa, hyalina, $7-9$ / 1,5-2, disticha; Paraphyses filiformes, hyalinae, apice paullulum crassiores. Jodii ope apex ascorum coerulescit.

a. Ad caules siccas Artemisiae vulg. in horto Eszterházyano Káposvár (Somogy) 1871 Lojka.

Edita in Rehm Ascomyc. 66.

b.? Ad lignum siccum. Gelsendorf (Stryj) Galiciae austr. Lojka.

38. Niptera cinerea (Batsch) Fuckel 1. c. f. a tr ocin er ea Cooke sec. Ellis in herb. meo.

ex s: Kunze f. sel. 179,382. Rabh. f. eur. 1643.

Asci clavati 24-30/5, 8 spori; Sporidia elongato-cuneiformia, 1 cellularia, hyalina, $6,1,5$, disticha. Paraphyses filiformes, apice vix incrassatae. Jodii ope apex ascorum coerulescit.

$\Lambda d$ caules siccas Dipsaci sylv. prope Lunkány in Hung. 1872 Lojka.

39. Niptora Ligni (Desm. sub Cenangium) Rehm.

s y 11: Trochila - De N. Mollisia - Karsten myc. fenn. I p. 204. (? Pyrenopeziza - Sace. f, Ven. nov. IV p. 33).

exs: Rehm Ascomyc. 413 (sub Tapesia fusca v. Myricariae). cfr. Beiträge des naturhist. Vereines zu Augsburg 1877 p. 60.

Perithecia parenchymatice fusca. Asci clavati, 8 spori $40-45 / 6$; Sporidia elliptica, recta vel subcurvata, obtusiuscula, 1 cellularia, hyalina, 6-9 2, disticha. Paraphyses filiformes, haud clavatae. Jod Ad lignum mortuum corylinum. Kaposvár (Somogy) 1872 Lojka sub 67.

40. Niptera plicata Rehm nov. spec. 1878.

exs: Rehm Ascomyc. 570. 
Perithecia solitaria vel gregaria, sessilia, primitus sphaeroidea, subclausa, dein urceolata, sicca plicata, fusco-cervina, margine tenui, albido, subdilacerato cincta ; disco livido albo, parenchymatice fuscidule contexta, $-1 \mathrm{~mm}$ diam., orbicularia. Asci clavati, apice incrassati, 8 spori, 45-60/6-9. Sporidia elliptica, plus minusve obtusa, recta vel subincurvata, 1 cellularia, hyalina, disticha, $9-12 / 2-3$. Paraphyses obtuse lanceolatae, plus minusve ascos superantes, $4-5$ mikr. diam., hyalinae. Jodii ope apex ascorum coerulescit.

Ad caules siccas Angelicae infra alpem Retyezát (Ilunyad) Transsylvaniae. 8/1877 Lojka

41. Niptera Polygoni (Cooke sub Peziza atrata f. -) Rehm 1871.

syn: Peziza (Mollisia) luctuosa Cooke.

c f r. Winter in Flora 1872 p. 542 . Cooke in Hedwigia 1875 p. 83 no 14 .

exs: Rehm Ascomyc $70 a, b, c$. Cooke f. brit. I 284. Thümen f. austr. 1011. Ellis n. am. f. 442. (? Rabh. f. eur. 1117, Phillips elv. brit. 174).

Sporidia elongato-cunoidea, obtusa, recta vei subcurvata, hyalina, 1 cellularia, $5-8 / 2,8$ disticha in ascis clavatis, apice incrassatis, 30--40/5--6; Paraphyses filiformes, 1,5-2 nikr. crassae, haud clavatae. Jodii ope apex ascorum valde coerulescit.

a. Ad caules putridas Polygoni in horto Esterházyano Kaposvár, (Somogy). 5/10 1871 Lojka.

Edita in Rehm Ascomyc. 70a.

b. ibidem prope Malomviz (Hunyad) Transsylvaniae 8/1872 Lojka sub 1885.

Edita in Rehm Ascomyc. $70 b$.

42. Niptera Vitis Rehın (sub Pyrenopeziza -) 1871!

c f r diagnosin cl. Winterii in Flora 1872 p. 526.

sy n: Peziza ampelina Passerini 1874!

exs: Rehm Ascomyceten 67. Rabh. f. eur. 1706

Sporidia obtuse conoidea, unicellularia, hyalina, $8 / 2,5,8$ disticha in ascis clavatis, apice incrassatis, 36 6. Paraphyses hyalinae, filiformes. Perithecia parenchymatice fusce contexta. Jodii ope apex ascorum coerulescit.

Ad corticem trunci Vitis viniferae in sylva Kislakensi prope Kaposvár (Somogy) 7/1871 Lojka sub 122. Videtur potius forma Nipterae atratae (Pers)!

Edita in Rehm Ascomyceten 67. 
43. Pseudopeziza rosella Relim nov. spec.

Perithecia sessilia, gregaria, minutissima, in maculis epidermidis flavidulis plerumque insidentia, orbicularia, plana, margine fuscidulo cincta, disco rosello, basi parenchymatice flavidule contexta, c. $0,2 \mathrm{~mm}$ diam. Asci clavati, apice incrassati, 8 spori, 45/9. Sporidia obtusa, oblonga, 1 cellularia, hyalina, disticha, 9/2,5-3. Paraphyses superne dichotomae et subincurvatae, haud incrassatae, laxae, hyalinae. Jodii ope apex ascorum violascit.

Ad caules putrescentes Aconiti Napelli. Arágyes infra alpem Retyezát (Hunyad) Transsylvaniae. 8/1874 Lojka.

44. Micropeziza Iridis Rehm nov. spec. 1873.

Perithecia sicca oblonga vel rotundata, plus minusve hemisphaerice contracta, humida concava vel explanata, sessilia, gregaria, subfusca, margine viridiflavo cincta, parenchymatice fusce contexta, disco fuscidulo, c. $0,3 \mathrm{~mm}$ diam. Asci clavati, apice incrassati, 50/9; 4-8 spori. Sporidia elliptico - elongata, haud acuminata, recta, 1 cellularia, nucleis magnis $1-4$ praedita, disticha, 18 21/4. Paraphyses filiformes, hyalinae, superne sensim - 3,5 mikr. incrassatae. Jodii ope apex ascorum coerulescit.

exs: Rehm Ascomyceten 161.

Ad caules et folia sicca Iridis spuriae prope Tököl in insula Danubii Csepel (Pest) Lojka sub 1988.

Edita in Rehm Ascomyc.

45. Habrostictis diaphana Rehm nov. spec.

Perithecia aurantiaca, primitus immersa, dein sessilia, plane ut in Calloria fusarioide, parenchymatice, dilute flavidule contexta, hyphis teneribus, hyalinis, brevibus insidentia. Asci clavati, apice incrassati, c. 50-70/9-12, $4-8$ spori; Sporidia obtuse oblonga, 1 - demum 2 cellularia, hyalina, $10-12 / 4-5$; transverse $1 \frac{1}{2} 2^{-}$ sticha. Paraphyses filiformes, snperne clavula elliptica, hyalina, c. 3 mikr. crassa instructae. Jodii ope apex ascorum violascit.

exs: Rehm Ascomyc. 118 (sub Calloria fusarioides var. alpigena), 210. Thümen f. austr. 1113, myc. un. 719.

Ad caules siccas Angelicae prope lacum Zenoga infra alpem Retyezát (Hunyad) Transsylvaniae c 6000’. Lojka 8/1873.

Edita in Rehm Ascomyc. 210.

46. Habrostictis ocellata (Pers. sub Peziza) Fuckel.

s y n : Stictis - Fr. T'ul. Peziza Lecanora Schm. et Kze. Stictis - Fr. syst. Propolis - et Populi De N. Phacidium Populi I asch. Ocellaria aurea Tul. O, parvula Speg. 
cfr Cooke hdb. p. 735. Fuckel symb. myc. Nachtrag I p. 326, II p. 47. (? Karsten myc. fenn. I p. 242. Jod -; Michelia VII p. 333 sporidia utrinque acutiuscula !)

ex s: Rabh. f. eur. 457. hb. myc. II 519. Fuckel f. rhen. 2368, 2669. Thümen myc. un. $276 a, 277$ b. Kunze f. sel. 171, 366. Spegazzini dec. myc. it. 103. Rehm Ascomyc. 265, 514 (n o n = Phillips elv. brit. 148).

Asci ovato-clavati, c. $120 / 15,8$ spori. Sporidia obtusa, elliptica, 1 cellularia, hyalina, $-27 /-10$, disticha. Paraphyses filiformes, granulatae, superne 2 mikr. crassae Epitheciumque flavum formantes, clava elliptica flavidula. Hypothecium flavum. Jodii ope apex ascorum violascit.

Ad ramulos siccos Cytisi austriaci prope Szent-Miklós in insula Csepel Danubii (Pest) 6/1873 Lojka sub 1994.

47. Calloria coccinella (Sommf. sub Peziza) Rehm.

s yn: Orbilia - Karsten myc. fenn. I p. 98

e f r Nyl. pez. fenn. p. 55. Saccardo f. Ven. nov. IV p. 30. Michelia I p. 428.

exs: Rehm Ascomyc. 71 (sub C. coccinella f. vinosella Nyl.) Exemplar juvenile! Asci clavati, 30/3-4. Sporidia, ut videtur, subglobosa, hyalina, monosticha. Paraphyses filiformes, clava rotunda coronatae. Hypothecium flavidulum. Jod - .

Ad lignum putridum in Galicia australi. 1869 Lojka sub 20.

48. Calloria fusarioides (Berk. sub Peziza) Fr. S. Veg.; Tul.

c f r. Nyl. pez. fenn. p. 57. Cooke hdb. p. 704. Karsten myc. fenn. I p. 96 (? Fuckel symb. myc. p. 282).

ex s: Rehm Ascomyc. 72. Spegazzini dec. myc. it. 26. Thümen myc. un. 277. Karsten f. fenn. 646. Zopf et Syd. myc. march. 90. Sacc. myc. Ven. 1501. Rabh. hb. myc. II 676. (? 20, 73 ; Cooke f. brit. II 381.) Moug. et Nestler stirp. vog. 396 (Dacrymyces) sub Tremella Urticae Pers.

Asci clavati, 8 spori, 50-55/10. Sporidia disticha, elongatoovata, 1 (-2) cellularia, recta vel subcurvata, hyalina, 9-10/4; Paraphyses parcae, filiformes, superne subincrassatae. Jod -.

Ad caules siccas Urticae dioicae in horto Esterházvano Kaposvár (Somogy). 5/1871 Lojka.

Edita in Rehm Ascomyc. 72 !

49. Calloria vinosa (Alb. et Schw. sub Peziza) Fr.

sy n: Orbilia - Karsten myc. fenn. I p. 101. (? Habrostictis rubra Fuckel symb. myc. p. 249 Nachtrag II p. 47). 
c f r Nyl. pez. fenn. p. 56. Saccardo f. Ven. nov. IV p. 30. Michelia I p. 63. Cooke hdb. p. 700. Dr. Winter in Flora 1872 p. 510. (n o n Fuckel symb. myc. p. 283 (sec. descriptionem paraphysum pertinet ad C. coccionellam).

ex s: Rehm Ascomyc. 17. Fuckel f. rhen. 2554 (sub Habr. rubra). Rabh. f. eur. 1412. (n o n Fuckel f. rhen. $2569=$ Peziza luteorubella Nyl. pez. fenn. p. 55, ne c Ellis n. am f. $142=$ Calloria occulta Rehm).

Hymenium rubidulum; Paraphyses filiformes, in clavam sensim abeuntes; asci clavati c. 30/6. Sporidia desunt.

Exemp'aria haud evoluta huc trahenda videntur.

Ad ramulos siccos Rosae caninae in valle Kolczvárensi (Hunyad) Transsylvaniae. 8/1873 Lojka.

50. Calloria vinosula Rehm nov. spec. 1872 !

Perithecia vix conspicua, minutissima, gregaria, sessilia, humida subconvexa, sicca concavula, flavidula, parenchymatice contexta. Asci ovato-elliptici, apice incrassati, 8 spori, 30/6-8. Sporidia disticha, elliptica, obtusa, 1 cellularia cun nucleis $2-4$, dein 1 septata, hyalina, 6-8/2. Paraphyses superne trifidae, articulatae, in clavam ellipticam, hyalinam, -3 mikr. crassam apice ampliatae ascosque superantes. Jod -. Kaposvár (Somogy) leg. Lojka.

a. ad ramum decorticatum Sambuci Ebuli.

b. ad caulem siccam Daturae Stramonii sub $117 b$.

51. Durella atrata (Hedw. sub Lichen) Rehm.

syn: Peziza - Wahlbg. Patellaria - Fr. syst. Lecanidion - Rabh. Peziza patellaria Pers. Lecidea saprophila Fw. Ucographa Lecanactis Mass. catagr. p. 4. Opegrapha Mass. symm. p. 64. Pragmopora-Körb. par. lich. p. 279.

c f r Nyl. pez. fenn. p. 67. Saccardo myc. Ven. p. 161. tab. XV f. 47. Fuckel symb. myc. p. 266 tab. IV f. 32 (n o n Cooke hdb p. 716).

ex s: Körber lich. sel. germ. 199. Anzi lich. rar. Ven. 96. Rabh. f. eur. 1612. Zwackh lich. 444. Saccardo myc. Ven. 263. Fuckel f. rhen 1118. Ellis n. am. f. 64. Erb. critt. it. 821. Rehın Ascomyc. 574 (? Phillips elv. brit. 90), - $\mathrm{n} 0 \mathrm{n}$ Conke f. brit. II 194 (sporidia $4-5$ cellularia, $21-30 / 4-4,5$ Jod) $=$ Durella atrella Rehm nov. spec.

Asci clavati, apice incrassati, c. $120 / 18,8$ spori, Sporidia elongato-clavata, obtusa, recta vel plerumque subcurvata, 6-8 cellularia, unaquaque cellula nucleo uno magno praedita, hyalina, 
- 45 9, disticha. Paraphyses filiformes, superne ramosac et in clavam ellipticam, -5 mikr. crassam, coeruleam exeuntes. Hypothecium nigrocoeruleum. Jod -

Ad caules Maydis putrescentes. Kaposvár (Somogy) 1872 Lojka. sub 113.

52. Durella commutata Fuckel symb. myc. p. 281.

exs: Rabh. hb. myc. II 509 (sub Peziza compressa).

Asci clavati, 8 spori, 45/6; Sporidia ovata, 1 cellularia, hyalina, superne disticha, $6-8 /-3$. Paraphyses filiformes, apice clava crassa fusca instructae. Jod -

Ad lignum decorticatum. Kaposvár (Somogy) 1872 Lojka sub 61.

53. Durella lecideola (Fr. S. V. Sc. sub Patellaria) Rehm.

s y n : Peziza - Fr. obs.

c f r Karsten myc. fenn. I p. 234. Nyl. pez. fenn. p. 64 (Jod +).

Asci clavati, crassi, 8 spori, c. $66 / 15$; Sporidia obtusa, elliptica, 4 cellularia, hyalina, demum fuscidula, $15-18 / 7-8$, disticha. Paraphyses septatae, clava fuscidula, $4-5$ mikr. crassa praeditae. Jod -

Ad lignum decorticatum. Kaposvár (Somogy) 1872 Lojka sub 152.

Dubium mihi est, an exemplar hungaricum ad speciem designatam pertineat. Excmplar originale hujus speciei mihi plane ignotum hucusque et reactio jodina minime congruit.

54. Karschia Strickeri Körb. par lich. p. 460.

syn: Patellaria - Saccardo in Michelia I p. 57. Poetschia -- Stein schlesische crypt. flora II 2 p. 224. (? Arthonia melaspermella Nyl. Patellaria lignyota Fr.)

ef $\mathrm{r}$ Grevillea VI p. 25. Flora 1872 p. 511, 1874 fragm. Arnoldii 16, Saccardo f. Ven. nov. IV p. 29.

cx s. Rehm Ascomyc. 21 (sub K. nigricans). Saccardo myc. Ven. 1201. Phillips elv. brit. 146 et Ellis n. am. f. 400 (sub Patellaria lignyota).

Asci clavati, apice incrassati, 8 spori, 30-36/12. Sporidia ovata, 2 cellularia, cellulis anisomeris, fuscidulis, disticha, 9-11/4, Paraphyses conglutinatae. Hypo- et Epithecium fuscidulum, Hymenium viridiflavum. Jodii ope asci coerulescunt.

Ad ramum querneum putridum in sylva Holobutowensi prope Brigidau (Sambor) Galiciae australis. Lojka. 8/1869.

55. Melaspilea Peltigerae Nyl. pez. fenn. p. 65 adn.

s y n: Pseudopeziza - Fuckel symb. myc. p. 291.

c f r Arnold in Flora fragm. 16. 
exs: Fuckel f. rhen. 1871. Rehm Ascomyc. 19. Arnold lich. exs. 700. Thümen myc. un. 1707.

Asci clavati, 8 spori, - $75 / 18$; Sporidia elongato-0roidea vel subfusiformia, $2-4$ cellularia, hyalina, disticha, $18-24,5-7$. Paraphyses filiformes, superne clavatae et Epithecium viride for mantes. Hypothecium flavescens. Jod -

Ad thallum vivum Peltigerae horizontalis L. prope Eperjes. Lojka 10/1869.

56. Cryptomyces Peltigerae Fuckel symb. myc. p. 253.

ex s: Fuckel f. rhen. 2069.

Exemplaria Hymenio prorsus inevoluto!

Ad saxa micaceo-schistosa vallis Kiolcvárensis prope rivulum "Riu sor" infra alpem Retyezát (Hunyad) Transsylvaniae. 8/1873 Lojka sub 2262-3.

57. Dermatea fascicularis (Alb. et Schw. sub Peziza) Fr.

syn: Peziza populnea Pers. Encoelia fascicularis Karsten.

c f r Karsten myc. fenn. II p. 217. Fuckel symb. myc. p. 278. Cooke hdb. p. 678. Nyl. pez. fenn. p. 15.

ex s: Rabh. hb. myc. II 620 (? f. eur. 721), Fuckel f. rhen 1128. Cooke f. brit. I 478. Phillips elv. brit. 17a, $b$. Thümen myc un. 663. Rehm Ascomyc. 301.

Asci clavati, apice subincrassati, $75 / 7-8,8$ spori. Sporidia oblonga, obtusa, recta vel subcurvata, hyalina, 1 cellularia, disticha, $12-15 / 3-3,5$. Paraphyses filiformes, apice sensim clavatae et fuscidulae. Epi- et Hypothecium viridi-fuscum. Jod -

a. ad corticem emortui Fraxini Orni in valle Kolczvárensi. (Hunyad) Transsylvaniae. 8/1873 Lojka.

b.? ad lignum putridum in valle "Riu sor" infra alpem Retyezát (Hunyad) Transsylvaniae. 9/1874 Lojka sub 3450.

Exemplaria prorsus inevoluta et dubia, ascis et sporidiis plane deficientibus! Perithecia purpureo tincta.

58. Dermatea Syringae Rehm nov. spec. 1873.

Perithecia plerumque gregaria, ex rimis epidermidis erumpentia, primitus globosa, dein irregulariter expansa, rugoso-marginata, fusca, 0,5-1,5 mm. diam. Asci clavati, 8 spori, $50-60 / 5$. Sporidia elliptica vel oblongato-oovidea, 1 cellularia, saepe 2 nucleata, hyalina, disticha, $8-9 / 2-2,5$. Paraphyses filiformes, superne clava fusca $45 \mathrm{mikr}$. crassa instructae Jod -

Ad ramum siccum Syringae vulgaris prope Plavisevicza com. Szörény in Hungaria 9,1872 Lojka sub 1001 B. 
59. Cenangium Cerasi (Pers. sub Peziza -) Fr. syst.

s yn: Dermatea - De N.; T'ul.

c f r Karsten myc. fenn. II p. 223 (J-!) Fuckel symb. myc. p. 267. Cooke hdb. p. 724 . Saccardo myc. Ven. p. 160.

ex s: Mougeot et Nestler stirp. Vog. 494. Rabh. hb. myc. II 517, f. eur. 1025. Badische crypt. 435. Fuckel f. rhen. 1127. Cooke f. brit. II 659 ( $\mathrm{J}$ !) Thümen myc. un. 1863. Phillips elv. brit. 193 Rehm Ascomyc. 421.

Asci clavati, $909-10,6-8$ spori. Sporidia oblonga vel elliptica, obtusa, recta vel subcurvata, 1 cellularia, plerumque nucleo 1 magno centrali instructa, hyalina, disticha, 15/4. Paraphyses filiformes in clavam c. 3 mikr. crassam, fuscam ampliatae. Hymenium et Hypothecium flavidulum. Jodii ope apex ascorum coerulescit.

Ad ramum siccum Cerasi. Kaposvár 1872 Lojka sub 133.

60. Cenangium Prunastri Fr. syst. V.

s y n : Dermatea - Fr. S. V. Sc.

c f r Fuckel symb. myc. p. 267. Cooke h(b. p. 724.

exs: Rehm Ascomyc. 213 (? Fuckel f. rhen. 1126), non Rabh. f. eur. 125.

Asci clavati, \& spori, 60-70/12. Sporidia oblonga vel elongato-ovata, obtusa, 1 cellularia, 1-2 nucleis magnis praedita, an demum 2 cellularia?, hyalina, disticha, 12-14/4. Paraphyses filiformes, superne furcatae. Epi- et Iypothecium viridulum, Hypothecium fuscum. Jod -

Ad ramos siccos Pruni domesticae prope Malomviz (Hunyad) Transsylvaniae. \&, 1873 Lojka.

Edita in Rehm Ascomyc. 213!

61. Tympanis amphiboloides Nyl. var. hy popodiza. Nyl. c f r Nyl. pez. fenn. p. 71. adn. p. 72. Karsten myc. fenn. II 1. 231 .

exs: ? Rehm Ascomyc. 358.

Asci clavati, crassi, - 100 10-15, 8 spori. Sporidia elliptica, subacutata, 4-8 cellularia, cellula media plerumque longitudinaliter semel divisa, hyalina, $18-24 / 3$, disticha. Praeterea repleti sunt asci granulis minimis. Paraphyses ramosae, articulatae, clava $5 \cdots 6$ mikr. crassa, fuscidula praeditae. Epi- et Hypothecium viride. Jod sub 55 .

a. ad ligna quercina prope Kaposvár (Somogy) 1872 Lojka

b. ad ligna pinea in Transsylvania. Lojka sub 2521.

62. Tympanis Pinastri Tul. carp. 
(? = Fuckel symb. myc. p. 269.)

s y n: Cenangium laricinum Fuckel symb. myc. p. 270.

c f r Nyl. pez. fenn. p. 73.

? Karsten myc. fenn. I p. 28 (nil dicit de ascis polysporis).

exs: Fuckel f. rhen. 2473 (sub C. laricinum), (? 1103 sub Triblidium). Phillips elv. brit. 145. Rabh. f. eur. 33 sub Lecanidion atrum); explr in Fuckel symb. myc. p. $266=$ Triblidium pithyum declaratum!. Rehm Ascomyc. 356. (? Rabh. 1231, Erb. critt. it. II 828, Moug. et Nestl. stirp. Vog. 587 p. p.).

Asci clavati, apice incrassati, 90/9, myriaspori ; Sporidia oblonga, hyalina, 2-2,5/1. Paraphyses filiformes, apice subcurvatae, articulatae et clava fuscidula -5 mikr. crassa instructae. Epi- et Hypothecium fuscum. Jod -

a. Ad abietum corticem prope Teplieska (Liptó) 8/1872 Lojka sub 1359, ibidemque Zatracsano infra alpem Dzurowa.

b. Ad ramulos pineos in Galicia a ustrali 1869 Lojka.

63. Heterosphaeria Patella (Tode sub Sphaeria penetrans « patella) Grev.

s y n : Sphaeria - Pers. Peziza fimbriata Chaill. Peziza Chailletii Pers. Peziza leucomela $\beta$ Umbelliferae DC. Peziza Ligustici DC. Phacidium Patella Fr. Tympanis - Wallr.

c fr Fuckel symb. myc. p. 266. Karten myc. fenn. I p. 222 (asci apice Jod - !) Saccardo myc. Ven. 160, Michelia I p. 262, 332. (? Cooke hdb. p. 754 propter sporidia 1 - 4 septata).

var. Lojka e Rehm 1871 !

s y n: Pyrenopeziza fuscoatra Hazslinszky Közlemen. 1873. p. 58. tab. II f. 10 ; Zool. bot. Vhdlgen 1873 p. 368.

c f $r$ Winter in Flora 1872 p. 510.

exs: Rehm Ascomyc. 15 a b, 209 (sub Pyrenopeziza -).

Perithecia parenchymatica, fusca disco pallido vel roseo. Asci clavati, 8 spori, 60/9-12. Sporidia oblonga, obtusa, recta, interdum subcurvata et intus granulata, 1 cellularia, hyalina, disticha, 12-15 4-5. Paraphyses filiformes, in clavam ellipticam, c. 2,5 mikr crassam, hyalinam exeuntes ascosque superantes. Jodii ope apex ascorum coerulescit.

a. Ad caules siccas Umbelliferarum prope lacum Zenoga infra alpem Retyezát (Hunyad) Transsylvaniae c. $6000^{\circ}$ alt. 8/1872 Lojka.

b. Ibidem prope Soborsin in Hungaria 7/1872 Lojka.

Differt sporidiis plerumque 2 cellularibus. Jod -

Edita ex loco a in Rehm Assomyc. 209! 
64. Lachnella corticalis (Pers. sub Peziza ) Fr. S. Veg. Sc. syn. Helotiuın-Karsten mye fenn I p. 159.

c f r Nyl. pez. fenn. p 27 f. 3, Fuckel symb. myc. p. 280. Saccardo myc. Ven. p. 161. Michelia I p. 472. Cooke hdb. p. 687.

exs: Fuckel f. rhen. 1120. Rabh. hb. myc. II 625. f. eur. 915. Thümen myc. un. 280. Phillips elv. brit. 119. Kunze f. sel. 562.

Perithecia prosenchymatice contexta, pilis simplicibus, septatis, glabris, fuscis, c. 3 mikr. crassis obsessa. Asci clavati, apice incrassati, c. $75 / 6,8$ spori. Sporidia elliptica, subacuta, recta, hyalina, 2 cellularia, disticha, 12-15/3. Paraphyses filiformes. Jodii ope apex ascorum coerulescit.

In sylva Stryhaniecensi prope Gelsendorf (Stryj) in Galicia australi ad truncum Populi putrescentem. 8/1869 Lojka sub 329.

65. Lachnella flammea (Alb. et Schw. sub Peziza) Fr.

s y n: Helotium-Karsten myc. fenn. I p. 157.

c f r Fuckel symb. myc. p. 280. Nyl. pez. fenn. p. 28.

ex s: Fuckel f. rhen. 1121. Rabb. hb. myc. II 22. Phillips elv. brit. 72. Thümen myc. un. 520. Rehm Ascomyc. 418.

Perithecia prosenchymatice contexta, pilis simplicibus, fuscis, obtusis, subglabris c. $4-5$ mikr. crass. obsessa. Asci clavati $75-80$ / $9-10,8$ spori. Sporidia oblonga, obtusa, recta vel subcurvata, 1 cellularia, hyalina, -24/4, disticha. Paraphyses filiformes, apice haud clavatae. Jod -

Ad ramum decorticatum Lonicerae? Kaposvár. (Somogy) 1871 Lojka sub 484 .

66. Triblidiopsis Pinastri (Pers. sub Peziza) Karsten myc. fenn. I p. 262.

s y n : Cenangium - Fr. syst. Phacidium - Fr. elench. Triblidium - Fr. S. V. Sc.

c f r Nyl. pez. fenn. p. 73. (n o n $=$ Fuckel symb. myc. p. 269 tab. IV f. 28)

exs. Karsten f. fenn. 557 (? Rabh. f. eur. 1026, 1103 sub Triblidium, 1140 sub Tympanis).

Exemplaria plane inevoluta sine ascis et sporidiis!

Ad ramulos pineos. „Válye Rásza" (Retyezát) in Transsylvania. Lojka sub 1723.

67 Phacidium Maydis Rehm nov. spec. 1873.

Perithecia gregaria, hemisphaerica, epidermide denigrata pustulatimque elevata immersa, dein in lacinias $4-6$ triangulares dilacerata et discum pallidum, orbicularem, planum aperientia, pa- 
renchymatice viridi-fusce contexta, c. $0,8-1 \mathrm{~mm}$. diam. Asci clavati, 8 spori, 60/7-8. Sporidia filiformia, ut videtur 8 cellularia et incellulas singulas haud difficiliter soluta, hyalina, parallele contorta, 30-36/1,5. Paraphyses filiformes, c. 2 mikr. crassae. Jod -

Ad caulem putrıdam Zeae Maydis. Kaposvár (Somogy) 1872. Lojka sub 112.

68. Aggrium rufum (Pers. sub Stictis) Fr.

c f r Cooke hdb. p. 733. Nyl. lich. scand. p. 250. Minks in Flora 1880 no 33.

exs: Anzi lich. it min. rar. 466. Phillips elv. brit. 142. Ravenel n. am. f. 314 . Ellis f. n. am. 450.

Sporidia ovata, nucleo 1 magno centrali praedita, 1 cellularia, hyalina, 12 6-7, 8 disticha in ascis clavatis. Hypothecium hyalinum. Paraphyses superne fuscidule conglutinatae. Jodii ope Hymenium coerulescit.

Ad ligna pinea decorticata circa lacum Zenoga infra alpem Retyezát (Hunyad) Transsylvaniae. Lojka sub 2409.

69. Coccomyces quadratus (Schmitt et Kunze sub Phacidium) Karsten.

s yn: Phacidium leptideum Fr. syst.

c fr Karsten myc. fenn. I p. 255. Fuckel symb. myc. p. 262.

ex s: Moug. et Nestl. stirp. vog. 984. (? Fuckel f. rhen. 1092. Thümen f. austr. 873).

Asci $-105 / 15$, \& spori Sporidia filiformia, pluricellularia, hyalina, c. $65 / 2,5$, parallela. Paraphyses filiformes. Jod -

Ad frutices Vaccinii Myrtilli prope Teplicska (Liptó) 8/1872 Lojka sub 1204.

70. Pleiostíctis propolidoides Rehm nov. genus et spec. 1880 !

Perithecia in ligni dealbati superficie primitus immersa, lirelaeformiter supremo ligni strato cincta, dein fere sessilia, primitus elliptica, dein rotundata, subplana, aperta, margine tenui, sublacerato, concolori cincta, atrofusca. gregaria, c. $0,8 \mathrm{~mm}$. diam.

Asci elongati, monospori. Sporidia oblonga, obtusa, hyalina, dein fuscidula, transverse 12-16, longitudinaliter 1-2 septata, 24-30/5-9. Paraphyses filiformes, superne c. 3 mikr. crassae, clavatae, fuscidulae, conglutinatae. Hymenium alt. c. 45 mikr. Hypothecium dilute fuscidulum. Jodii ope Hymenium coerulescit, dein vinose rubescit.

Ad ramulos Abietum in regione "Arágyes" infra alpem Retyezát (Hunyad) Transsylvaniae 8/1873. Lojka sub 2430. 
Genus Stictideum valde eximium ascis monosporis, sporidiis oblongis, multicellularibus.

Etab cl. Arnoldio allatum speciminulum ex alpibus editioribus prope Kühtei (Oetz) ad lignum decorticatum Pini Cembrae in herbario meo depositum est!

71. Robergea unica Desm. 1847.

sy n: ? Cryptella - Quell. Tuberculostoma lageniforme Sollm. Valsa-Currey. Ostropa cubicularis Fuckel. (n o n Sphaeria - Fr.).

c fr Cooke hdb. p. 829. Fuckel symb. myc. p. 92. Hedwigia 1868 no 3 adn. Michelia I p. 252,257.

e x s. Rabhorst f. eur. 765 (sub. 'Tub. lageniforme), Fuckel f. rhen. 2035 (Ostropa cubicularis), 'Thümen f. austr. 1156 (Schmitzomia radiata), Riehm Ascomyc. 31 sub Ostropa cubicularis.

Asci cylindracei, apice valde incrassati, 8 spori. Sporidia filiformia, multicellularia, hyalina, c. 200/2,5, parallela. Paraphyses filiformes, tellerrimae Jod -

Ad ramulos Syringae vulgaris (ibidem spontaneae) prope Cetati Boli (Hunyad) Transsylvaniae. S/873 I ojka sub 2094.

72. Colpoma quercinum (P'ers. sub Hysterium) Wallr.

s y n: Cenangium -- Fr. Clithris- Karsten myc. fenn. I p. 221.

c fr Fuckel symb. myc. p. 257. Cooke hdb. p. 765. Saccardo myc. Ven. p. 157.

exs: Moug. et Nestler stirp. vog. 367. Rabh. hb. myc. II 449. Badische crypt. 645. Fuckel f. rhen. 760. Cooke f. brit. If 197. Thümen myc. un. 369 (? f. austr. 508). Saccardo myc. Ven. 717, 259. Karsten f. fenn. 328 (sub Cenangium). Rehm Ascomyc. 27.

Asci elliptici, stipitati, — 135/9, S spori. Sporidia filiformia, multicellularia, hyalina, paral'ela, extus ascos convoluta, c. 90/1,5. Paraphyses filiformes, tenerrimae, superne hamulatae. Jod -

Ad ramulos querneos siccos, Kaposvár 1871 Lojka sub 148.

73. Lophium mytilinum Pers. (sub Hysterium -) Fr.

s y 1 :? Lophidium mytilinellum Karsten myc. fenn. II p. 247.

c f r Fuckel symb. myc. p. 83. Cooke hdb. p. 766. Saccardo f. Ven. IV p. 28. (n o n Karsten myc. fenn. II p. 245) Mytilinidion aggregatum); Revue myc. 11 p. 49.

exs: Rabh. hb. myc. II 714. Fuckel f. rhen. 762.

Asci cylindracei, 8 spori. Sporidia filiformia, multicellularia, hyalina, parallela, c. 100/2. Paraphyses filiformes, tenerrimae. Jod Ad ramulos pineos siccos in Galicia australi 8/1869. Lojka sub V 1869. 


\section{Mytilinidion lineare Rehm nov. spec.}

ex s: Rehm Ascomyc. 216.

Perithecia sessilia, in maculas atras, subrotundas, c. $1-3 \mathrm{~mm}$ diam. coacervata, minutissima, mytiliformia, atra, carbonacea, sublinearia. Asci cylindraceo-clavati, apice incrassati, 8 spori, 60/6 Sporidia elongato-fusoidea, recta vel subcurvata, didyma, fuscidula, disticha, 12/2,5. Paraphyses ramosae. Jod -

Ad frustula ligni pinei putrescentis in valle Zserelen infra montem Domuglad prope Thermas Herculis com. Szőrény in Hung. 4'1874. Lojka sub 2586.

Edita in Rehm Ascomyc. 75.

75. Mytilinidion tortile (Schwein. syn. p. 50 sub Hysterium). c f r Saccardo f. Ven. IV p. 27.

exs: Thümen myc. un. 74. Rehm. Ascomyc. 269 [n o n Saccardo myc. Ven. 1174 (sporidia dimidio minora!]

Sporidia oblonga, obtusa, 6 cellularia, plus minusve nucleata, ad septa vix constricta, recta, fusca, $24 / 9 ; 81 \frac{1}{2}$ sticha in ascis cylindraceo-clavatis, crassis c 140/15. Paraphyses paucae, superne ramosae. Jod -

Ad corticem trunci Juniperi Sabinae; Petrila (Hunyad) in Transsylvania. 1873 Lojka sub 2126.

Species hucusque tantum ex America septentr. cognita!

76 Lophodermium arundinaceum (Schrad. sub Hysterium) Chev.

s y 11 : Hysterium culmigenum Fr., H. apiculatum Fr., gramineum Moug., H. seriatum Lib., caricinum Rob., Lophodermium Actinothyrium Fuckel symb. myc. Nachtrag III p. 28.

e f r Fuckel symb. myc. p. 257. Cooke hdb. p. 763. Karsten myc. fen. II p. 242. Saccardo myc. Ven. p. 157. Michelia I. p. 252.

exs: Fuckel f. rhen. 737, 738, 739, 740, 741, 1751, 2557, 2675. Cooke f. brit. II 300. Rabh. hb. myc. II 444. a, b, 723. f. eur. 1226 (? 1613). Thümen myc. un. 77, 471, 661. Saccardo myc. Ven. 1173. 1280. Moug. et Nestler stirp. vog. 655. (? 368). Kunze f. sel. 160, 161, 372. Zopf et Sydow inyc. march. 25. Ellis n. am. f. 465. Rehm Ascomyceten 271 (sub f. culmigenum), 58) (f. Secalis).

Asci clavati, apice conico-truncati, $75 / 9-10,8$ spori. Sporidia filiformia, nucleata, subrecta, hyalina, 1 cellularia, 8 parallele sita. Paraphyses apice subincrassatae, vix hamulatae. Jod -

Ad folium gramineum prope Tököl in insula Danubii Csepel (Pest.) 1873 Lojka sub 1988. 
77. Hysterium acuminatum $\mathrm{Fr}$. syst.

c f r Fuckel symb. myc. p 259. Rabh. D. Crypt. I. p. 154. f. a l p in u in Rehm.

ex s: ? Fuckel f. rhen. 750. Rehm Ascomyc. 125 sec. Minks.

Asci cylindracei, 8 spori. Sporidia oblonga, 4 cellularia, medio, minus ad cetera septa subconstricta, interdum una cellularum mediarum longitudinaliter septata, recta, fusca, $15-18 / 6-7$, monosticha Paraphyses ramosae. Jod -

Ad truncum putridum Pini circa lacum Zenoga infra alpem Rétyezát (Hunyad) Transsylvaniae. Lojka sub 2407.

Mihi plane dubium est, an haec species certe sit Hysterium acuminatum Fr. Exemplar Fuckelii cum meis in Ascomyc. 125 ex Alpibus editioribus Tyrolensibus editis haud congruit.

78. Hypoderma virgultorum DC. fl. fr. 5 p. 165 ; Duby hyst. 41. c f r Fuckel symb. myc. p. 258. Cooke hdb p. 761. Saccardo myc. Ven. p. 158. Michelia I p. 57, 514.

f. R u bi.

ex s: Moug. et Nestl. stirp. vog. 564 (sub Hysterium Rubi). Rabh. hb. myc. II 443, f. eur. 1618. Fuckel f. rhen. 757. Cooke f. brit. II 460. Thumen myc. un. 180. Saccardo myc. Ven. 720. Kunze f. sel. 162. Rehm Ascomyc. 362.

Asci superne elliptici, longe stipitati, \& spori; Sporidia elliptica vel fusiformia, subobtusa, recta vel subcurvata, primitus 1 cellularia cum nucleis 2 magnis, dein 2 cellularia, hyalina, $2-3$ sticha. Paraphyses filiformes, apice hamulatae. Jod --

Ad ramulos siccos Rubi Idaei infra "Theu ursului" prope Lunkány in Hungaria. 7/1872. Lojka sub 1883a.

79. Glonium lineare (Fr. sub Hysterium) De N.

c f r Fuckel symb. myc. p. 260. Cooke hdb. p. 760. Saccardo myc. Ven. p. 159 tab. XVI f. 13-15. Karsten myc. fenn II p. 237. Revue myc. 11 p. 237.

exs: Rabh. f. eur. 841. Fuckel f. rhen. 754 (sub Gl. confluens), 2560. Cooke f. brit. II 457. Saccardo myc. Ven. 948 (sub Gl. lineare var. angustissimum De N.). Rehm Ascomyc. 365. Ellis n. am. f. 463.

Exemplar nimis vetustum cum singulis sporis.

Ad lignum decorticatum Coryli (?). Kaposvár (Somogy). 1872 Lojka sub 43.

80. Pseudographis Carestiae (De N. sub Blitridium) Rehm. ef $\mathrm{r}$ De N. in erb. critt. it. II 92. Hedwigia 1868 p. 121. 
syn: Phacidiopsis alpina Hazsl. in bot. Vhdlgen 1873 (non = Peziza macrospora Karsten, Nyl. cfr Karsten myc. fenn. I. p. 235. Nyl. pez. fenn. p. 66, quae = Patellaria Carestiae declaratur!)

exs: Rabh. f. eur. 1224 (sub Blitridium).

Perithecia senilia, ascis subevanidis. Sporidia obtusa, oblonga, 8 cellularia, hyalina, 18/9. Paraphyses filiformes, guttulatae, rigidae. Hypothecium flavidulum. Jod -

Ad ramulos siceos, pendulos Rhododendri myrtifolii. Retyezát in Transsylvania 1872; leg. Lojka sub 1849.

81. Pseudographis pinicola (Rebent. sub Peziza) Rehm.

s yn: Heterosphäria - Fr. S. V. Sc. Triblidium-Cooke in Grevillea IV tab. 67 f. 8 . (n o n Crumenula pinicola Karsten myc. fenn. I p. 210.)

e fr Nyl. pez. fenn. p. 5, 77 adn.

ex s: Rabh. f. eur. 124. Schweiz. crypt. 627 (sub Hysterium elatinum). Rehm Ascomyc. 24 (sub Hysterium pinicolum).

Asci clavati, $150 / 15,8$ spori. Sporidia elliptica, subobtusa, recta vel navicularia, (2 ) plerumque 8 (rarius 10 ) cellularia, hyalina, 30/9, disticha. Jodii ope episporium valde coerulescit.

Ad corticem Pinorum vallis "Riu sor" infra alpem Retyezát in Transsylv. Lojka sub 1864 .

82. Xylographa parallela (Ach. sub Opegrapha -) Fr. S. V. Se.

s y 11 : Hysterium - Wahlbg. Stictis - Sommf., Fr. syst.

ef $\mathrm{r}$ Th. Fries lich. aret. p. 212. lich. scand. p. 638 Krempelhuber Bay. Lich. p. 266. Nyl. lich. scand. p. 250. Koerb. par. lich. 275. Cooke hdb. p. 736. Fuckel symb. myc. p. 252. Stein schles. Flechtenflora p. 261. Saccardo f. Ven. V p. 184. Minks in Flora 1880 no 34.

ex s : Koerb. lich. sel. germ. 257. Anzi lich. Lang. 346. Arnold lich. exs. 244 $a, b$. Fuckel f. rhen. 2672. Kunze f. sel. 368. Rehm Ascomyc. 124.

Asci clavati, 8 spori, $60-70 / 18$; Sporidia plus minusve ovata vel elliptica, 1 cellularia, nucleo 1 magno centrali, rarius nucleis 2 praedita, hyalina, 12-15/6, disticha. Paraphyses filiformes, articulatae et in clavam fuscam c. 5 mikr. diam. exeuntes. Hypothecium hyalinum, Epithecium fuscum. Jodii ope Hymenium coerulescit.

a) Ad asseres regionis Tielhavy prope Teplicska (Liptó). 8/1872 Lojka sub 1242. 
b) ad lignum decorticatum Pini Pumilionis. Válye Rásza infra alpem Retyezát Transsylvaniae. 8/1872 Lojka sub 1728.

83. Xylographa spilomatica (Anzi symb. p. 20 sub Agyrium) Th. Fr. lich. scand p. 639.

syn: X. minutula Körb. par. lich. p. 276. X. corruscans Norm. bot. not. 1872. p. 34 .

c f r Stein schles. Flechtenflora p. 261. Minks in Flora 1880 no 34.

exs: Anzi lich. lang. no 385 (sub Agyrium rufum). Arnold lich exs. 563 et Rehm Ascomyc. 123 (sub. X. minutula).

Asci clavati, apice incrassati, 8 spori, c. 66/15. Sporidia suovata vel oblonga, obtusa, nucleo 1 magno centrali praedita, 1 cellularia, hyalina, $9-12 / 5$, disticha. Paraphyses filiformes, superne crassiores et fuscidulae, conglutinatae. Hypothecium hyalinum. Jodii ope Hymenium coerulescit.

Ad truncum abietinum putridum in valle "Riusor" infra alpem Retyezát Transsylvaniae. 8/1872 I_ojka sub 1874.

84. Helvella albipes Fuckel symb. myc. p. 334. t. 5 f. 2.

e fr Cooke mycogr. p. 198 tab. 93 f. 336.

exs: Fuckel f. rhen. 1240 (f. minor.)

Exemplar altitudinis $7 \mathrm{~cm}$ ! Asci cylindracei, 4 spori. Sporidia oblonga, obtusa, nucleo magno 1 centrali instructa, 1 cellularia, glabra, monosticha, 18/12. Paraphyses filiformes, superne articulatae et c. 4 mikr. diam. clavatae. Jod -

Ad terram arenosam sub Populo. Szent-Miklós in insula Danubii Csepel (Pest). Lojka 6/1873 sub 1997.

85. Ascophanus carneus (Pers. syn. p. 676 sub Ascobolus) Boudier mem. p. 60 pl. 12 f. $48-49$.

syn: Peziza - Karsten myc. fenn. I p. 60. Ascobolus Cooke hdb. p. 60. Ascophanus saccharinus Boud. mem. p. 6.

c fr Nyl. pez. fenn. p. 86. Fuckel symb. myc. p. 288. Michelia I p. 70,236 , II p. 77.

ex s: Fuckel f. rhen. 1857. Rabh. f. eur. 625 (sub Peziza testacea), 14.49. Thümen f. austr. 1012. Cooke f. brit. II 398. Ravenel f. amer. 176. Phillips elv. brit. 197. Rehm Ascomyc. 2 (? 425, videtur Peziza difformis Karsten myc. fenn. I p. 61, Nyl. pez. fenn. p. 85, syn? Ascobolus saccharinus Berk., Cooke hdb. p. 731.)

Asci clavati, 8 spori, 100/21-24. Sporidia oblonga, obtusa, 1 cellularia, hyalina, $-18 / 10$, disticha. Paraphyses filiformes, subhyalinae, apice sensim -7 mikr. clavatae. Jodii ope Hymenium valde coerulescit. 
a. Ad fimum vaccinum prope Gelsendorf (Stryj) Galiciae australis. 1869 Lojka.

b. ibidem Kaposvár (Somogy). 1871 Lojka sub 137.

$c$. ibidem. In ostio antri prope Ponor-Ohába (Hunyad) Transsylvaniae. 8/1875 Lojka.

d. ibidem Transsylvania 1872 Lojka sub 1876 p. p.

$e$. ad laminas papyri putridas in horto Esterházyano Kaposvár (Somogy). 10/18`1 Lojka sub 129.

86. Ascophanus granuliformis (Crouan sub Ascobolus) Boudier mem. p. 55.

s yn : Peziza - Karsten myc. fenn. I p. 50.

cf r Cooke hdb. p. 729. Michelia II p. 235 (? Fuckel symb. myc. p. 288.)

exs: Rabh. f. eur. 782. Rehm Ascomyc. 104 (? Fuckel f. rhen. 1850 , sporae majores!)

Asci clavati, 90/12, S spori. Sporidia ovata, obtusa, 1 cellularia, nucleo 1 centrali magno instructa, glabra, hyalina, $12-$ $15 / 6-7$, disticha. Paraphyses filiformes, apice subincrassatae, p. p. incurvatae. Jod -

Ad fimum vaccinum. Malomviz (Hunyad) Transsylvaniae. 8/1872 Lojka sub 1879.

87. Ascophanus pilosus (Fr. sub Ascobolus) Boud. mein. p. 64.

s y n: Peziza equina Müll., diversicolor Fr. syst., cervina Pers. Ascobolus papillatus Wallr., ciliatus Schmitt.

c f r Nyl. pez. fenn. p. 20, 86 adn. Karsten myc. fenn. I p. 72, 73. c. var. ciliata Schm. Cooke hdb. p. 731 (sub ciliatus).

exs: Rabh. hb. myc. II 426 (sub papillatus). Fuckel f. rhen. 1860 (subciliatus), 1133 (papillatus). Rabh. hb. myc. II 425 (ciliatus). Cooke f. brit. II no 190 et Phillips elv. brit. 97 (ciliatus). Kunze f. sel. 564. Rehm Ascomyceten $103 a, b$.

Asci clavati, 8 spori, c. 100/30. Sporidia obtusa, elliptica vel oblonga, glabra, 1 cellularia, hyalina, $21 / 9$, dsticha. Paraphyses filiformes, haud clavatae. Pili peritheciorum rigidi, acutati, simplices, non septati, glabri, hyalini, basi diam. 15-20 mikr. Jod -

Ad fimum vaccinum circa introitum antri prope Ponor-Ohába (Hunyad) Transsylvaniae. 8/1875 Lojka.

88. Saccobolus Kerverni (Crouan sub Ascobolus) Boudier mem. p. 39 pl. VIII f. 18.

c fr Fuckel symb. myc. p. 287. Cooke hdb. p. 729. Michelia II p. 234. 
syn: ? Accobolus glaber Karsten myc. fenn. I. p. 78 (cfr $\mathrm{Nyl}$. pez. fenn. p. 84.

exs: Fuckel f. rhen. 1846. Rabh. f. eur. 1313. Cooke f. brit. II 399 (delineatio non quadrat). Rehm Ascomyc. 166.

Asci ovato-clavati, 8 spori, c. 70/18. Sporidia obtusa, elliptica, 1 cellularia, fuscoviolacea, glabra, $21-24 / 9-12$, in medio asci specifice conglobata; Paraphyses filiformes, articulatae, superne clavatae. Hymenium flavidulum. Jodii ope Hymenium coerulescit.

a. ad fimum vaccinum prope Malomviz (Hunyad) Transsylvaniae. 8/1872 Lojka.

b. ibidem Kaposvár in Hungaria. Lojka 1871 sub 176.

c. ibidem Bandrów in Galicia australi 1879 Lojka.

89. Saccobolus violascens Boud. mem. p. 40 tab. 8 f. 19.

syn: Ascobolus glaber Fuckel symb. myc. p. 287 (non Cooke hdb. p. 728 nec Nyl. pez. fenn. p. 83, exs. Rabh. f. eur. 778, nec $=$ Karsten inyc. fenn. I p. 78) ? Ascobolus versicolor Karsten inyc. fenn. I p. 79.

exs: Fuckel f. rhen. 1134. Phillips elv. brit. 48 (videtur). Rehm Ascomyc. 165.

Asci ovato-clavati, 8 spori, c. 66/15. Sporidia elliptica, subobtusa, fusco-violacea, glabra, 1 cellularia, $15-17 / 7-8$, in medio asci specifice conglobata. Paraphyses filiformes, superne clava c. 3 mikr. crassa, flavidula instructae, articulatae. Jodii ope Hymenium coerulescit.

Ad finum vaccinum. Singlér prope Eperjes. 10/1869 Lojka. (Exemplaria manca!)

90. Saccobolus depauperatus (Berk. et Br. sub Ascobolus).

syn: Ascobolus Kerverni Karsten myc. fenn. I p. 78 .

c f r Cooke hdb. p. 729.

exs : Rabh. f. eur. 780 .

Asci ovato-clavati, 8 spori, c. $40 / 18$. Sporidia elliptica, demum fusca, 1 cellularia, glabra, $12 / 5$, in medio asci specifice conglobata. Paraphyses conglutinatae, apice clavatae et flavidulae. Jodii ope Hymenium coerulescit.

Ad fimum vaccinum prope Malomviz (Hunyad) Transsylvaniae. 1882 Lojka.

Speciminulum huc trahendum videtur!

91. Ascobolus furfuraceus Pers.

s yn: Peziza stercoraria Bull P. fusca Huds.

e fr Nyl. pez. fenn. p. 83. Fuckel symb. myc. p. 286. Cooke 
hdb. p. 727. Janczewski in Hofmann myc. Bericht III p. 94. Boudier mem. p. 29 pl. 6. Karsten myc. fenn. I p. 76. Michelia I p. 69, II p. 233, VII p. 258 (f. nudus Kickx).

ex s: Fuckel f. rhen. 1132. Rehm Ascomyc. $1 a, b$; Cooke f. brit. II 189. Phillips elvell. brit. 46. Ravenel f. am. 312.

Asci elliptico-clavati, 8 spori, c. 105/27. Sporidia elliptica, fuscoviolacea, episporio longitudinaliter, striaeformiter lacerato, 1 cellularia, - 24/10, disticha. Paraphyses filiformes, c. 2 mikr. crassae, superne vix clavatae. Epithecium flavidulum. Jod - . (interdum asci leviter coerulescunt !)

a) Ad fimum vaccinum. Malomviz (Hunyad) Transsylvaniae. 8/1872 Lojka sub 1878 .

b) ibidem. Válye Rásza infra alpem Retyezát (Transsylvania). 8/1872 Lojka sub 1869.

c) ibidem prope Gelsendorf (Stryj) in Galicia australi 1869 Lojka.

d) ibidem circa introitum antri prope Ponor-Ohába. (Hunyad) Transsylvaniae. 8/1875 Lojka (Exemplaria manca!).

92. Ascobolus immersus Pers.

syn: Ascobolus macrosporus Crouan.

c f r Boudier mem. p. 37. Nyl. pez. fenn. p. 84. Cooke hdb. p. 728. Fuckel symb. myc. p. 286 (immersus et macrosporus). Karsten myc. fenn. I p. 79. Michelia I p. 234

ex s: Fuckel f. rhen. 1847, 1848. Rehm Ascomyceten 102 $a, b$, (? Rabh. f. eur. 779). Cooke f. brit. II 397 sec. delin.

Asci ovales, $180,100,8$ spori. Sporidia elliptica, obtusa, demum violacea, episporio longitudinaliter, striaeformiter dilacerato, 1 cellularia, disticha, 48/30. Paraphyses filiformes, flavidulae, conglobatae. Jodii ope Hymenium coerulescit.

Ad fimum vaccinum in Galicia australi. 1869. Lojka. 


\section{Pyrenomycetes.}

1. Hypoxylon fuscum (Pers. sub Sphaeria) Fr. S. Veg.

s yn: cfr apud Nitschke pyren. I p. 35.

ctr Fuckel symb. myc. p. 234. Cooke hdb. p. 796. Saccardo myc. Ven. p. 152. f. it. del. 569. Karsten myc. fenn. I p. 39.

ex s: Moug. et Nestler stirp. vog. 178. Schweiz. crypt. 530. bad. crypt. 151. Rabh. f. eur. 628. Fuckel f. rhen. 1054. Plowright sphaer. brit. I 20 Thümen f. austr. 664. myc. un. 367 (? 871 ). Saccardo myc. Ven. 674 (675 ?). Cooke f. brit. II 467. Rehm Ascomyc. 221. Ellis f. n. am. 678 (J-) (? Ravenel f. am. 184 J-, Thümen myc. un. 1861).

Asci cylindracei, 90-100/6, 8 spori. Sporidia elliptica, plerumque subcurvata, 1 cellularia cum $1-2$ nucleis magnis, hyalina, dein fusca, monosticha, 10-12/6-7. Paraphyses articulatae, ramosae. Jodii ope apex ascorum coerulescit.

Ad ramum siccum (Coryli ?). Kaposvár (Somogy) 1872 Lojka sub 70 .

\section{Nummularia Bulliardi Tul.}

s yn: Sphaeria nummularia DC. Hypoxylon - Bull.

c f r Nitschke pyren. I p. 60. Fuckel symb. myc. p. 236. Cooke hdb. p. 798.

ex s: Moug. et Nestler stirp. vog. 374. Fuckel f. rhen. 1062. Ellis f. 11. am. 85 (? sine hymenio in herb. meo.)

Asci evanidi. Sporidia elliptica, 1 cellularia, plerumque nucleis 2 magnis apicalibus instructa, fusca, - 12/7. Exemplar valde mancum!

Ad ramum fagineum. Kaposvár (Somogy) 1872 Lojka sub 65.

3. Poronia punctata (L. sub Peziza) Fr. S. V.

s y n: Sphaeria - Fr. syst. Sph. Poronia Pers.

c f r Nitschke pyren. I p. 19. Cooke hdb. p. 791. Karsten 
myc. fenn. I p. 36. Hansen champ. sterc. Dan. p. 14 (ubi cit. sy n. Peziza coriacea Bull = Patellaria Fr. = Lecanidion Rabh.)

ex s: Moug. et Nestler stirp. vog. 958. Rabh. hb. myc. II 45, f. eur. 2020. Cooke f. brit. I 468. II 213. Plowright sphaer. brit. II 9. Rehm Asconyc. 168.

Asci cylindracei, apice truncati et incrassati, 100/18, 8 spori. Sporidia elliptica, fusca, 1 cellularia, massa gelatinosa tenui cincta, 18-24/9; plerumque 1 sticha. Paraphyses ramosae, articulatae, $3-5$ mikr. crassae. Perithecium prosenchymaticum. Jodii ope apex ascorum valde coerulescit.

Ad fimum vaccinum prope Tököl in insula Danubii Csepel (Pest). 6/1873. Lojka sub 1989.

Edita in Rehm Ascomyc.

4. Valsaria insitiva Ces. et De N.

sy n : Sphaeria - Tode. Diatrype - Fr. S. V. Melogramma rubricosum Fr. Tul. Myrmaecium - Nitschke.

c fr Fuckel symb. myc. p. 227. Thümen Beiträge II p. 29. Saccarlo myc. Ven. p. 148 tab. XV f. 5-10, f. Ven. IV p. 20. $\mathrm{V}$ p. 183 (declarat V. rubricosam et insitivam esse diversas species!), f. it. del. 640. Michelia I p. 31.

a. f. quercicola Fuckel.

ex s: Fuckel f. rhen. 1821. Rehm Ascomyc. $170 a, b$. Rabh. f. eur. 2112 (? 1128).

Asci cylindracei, 8 spori, - 120/10-12. Sporidia biscoctiformia, nempe 2 cellularia, medio plusminusve constricta et utraque cellula nucleo 1 magno instructa, episporio saepe scabro, hyalina, dein fusca, monosticha, $-15 /-8$; Paraphyses tenerae, filiformes, guttulatae. Jod -

Ad ramum quercinum siccum. Toponár (Somogy) in Hungaria 1872 Lojka. sub 175.

b. f. viticola Fuckel symb. myc. p. 228

exs. Fuckel f. rhen. 1822.

c f r Myrmaecium lophiostomum Hazslinszky in Közlem. 1873 p. 55 .

Ad sarmenta sicca Vitis vinif. Kaposvár (Somogy) 1872. Lojka sub 147.

c. f. S y ring a e.

Ad ramulos siccos Syringae vulg. spontanee crescentis prope Plavisevicza in Hung. 9/1872 Lojka sub 1001.

d. f. Viburni. 
Ad ramos siccos Viburni Opuli in horto Esterházy ano Kaposvár (Somogy). 5/1871 Lojka.

5. Melogramma spiniferum (Wallr. sub Sphaeria) DN.

s yn: Diatrype podoides (Sphaeria-Wallr.) Fr. S. V.

c fr Fuckel symb. myc. p. 226. Saccardo myc. Ven. p 147. tab. XIV f. 68-69; f. it. del. 541. Michelia II p. 66.

exs: Fuckel f. rhen. 1000 (sub Bertia). Kunze f. sel. 153 Saccardo myc. Ven. 237. Moug. et Nestler stirp. vog. 1074 (sub) Sphaeria podoides Pers.! Spegazzini dec. myc. 93. Thümen myc. un. 1860.

Asci cylindraceo-clavati, 8 spori, 180/14. Sporidia dactyloidea, obtusa, subcurvata, 8 cellularia, cellulis finalibus hyalinis, ceteris fuscis, $-66 / 6$, disticha. Paraphyses hyalinae, ? ramosae, guttulatae, c. 3 mikr. crassae. Jod -

Ad truncum fagi emortuum. Kaposvár (Somogy). Lojka 1872 sub 65 .

6. Diatrype Stigma (Hoffm. sub Sphaeria) Fr.

syn: Stictosphaeria Hoffmanni Tul.

c fr Nitschke pyren. I p. 65. Fuckel symb. myc. p. 230. Cooke hdb. p. 811. Saccardo myc. Ven. p. 150, f. it. del. 464. Karsten myc. fenn. I p. 150.

ex s: Mougeot et Nestler stirp. vog. 372, 373 (sub Sphaeria decorticans). Rabh. hb. myc. II 529, f. eur. 820. Fuckel f. rhen. 1043 (1044? propter sporas minores). Plowright sphaer. brit. I 32. Thümen f. austr. 763. Fuckel f. rhen. 2263. Cooke f. brit. II 217. Saccardo myc. Ven. 943, 944. Rehm Ascomyc. $220 a, b$ (? Ravenel f. am. $359 a, b$. Ellis n. am. f. 491.)

Asci fusiformes, longe stipitati, 8 spori, pars sporifera c. 45 mikr. long. Sporidia disticha, oblonga, obtusa, subcurvata, flavidula, 1 cellularia, 9-11/2,5. Paraphyses filiformes. Jod -

Ad ramos siccos Pruni domesticae prope Malomviz (Hunyad) Transsylvaniae. 8:1873 Lojka.

Edita in Rehm Ascomyc. $220 a$.

7. Eutypa spinosa (Pers. sub Sphaeria -) Tul.

s y n: Valsa - Nke pyren. I p. 127. Diatrype Berengeriana Sacc.

c f r Fuckel symb. myc. p. 214. Michelia II p. 51.

ex s: Fuckel f. rhen. 1050. Rabh. f. eur. $936 a$, $b$. Moug. et Nestler stirp. vog. 376. bad. crypt. 831. Cooke f. brit. II 675 .

Asci fusiformes, 8 spori. Sporidia oblonga, obtusa, 1 cellularia, recta vel subcurvata, dilute flavidula, 5/2,5, disticha. 
Ad lignum fagineum in regione Turkului infra alpem Retyezát (Hunyad) Transsylvaniae. 7/1872 Lojka sub 1616.

8. Eutypa lejoplaca (Fr. syst. sub Sphaeria, S. Veg. Sc. sub Diatrype) Nitschke pyren. I p. 151.

c f r? Cooke hdb. p. 800.

exs: Fuckel f. rhen. 1047. Plowright sphaer. brit. 13,14. (? Ellis f. n. am. 679).

Asci fusiformes, stipitati, c. $45 / 6,8$ spori. Sporidia oblonga, obtusa, subcurvata, 1 cellularia, dilute flavidula, disticha, 7-8/2,5. Paraphyses filiformes, crassae. Jod -

Ad sarmenta putrida Vitis viniferae prope Kaposvár (Somogy) 5/1871. Lojka.

9. Eutypa flavovirens (Hoffm. Pers. Fr. syst. sub Sphaeria) Tul.

s y n : Valsa - Nke pyren. I p. 141. Diatrype - Fr. S. Veg.

c fr Fuckel symb. myc. p. 213. Cooke hdb. p. 799. Karsten myc. fenn. II p. 128. Saccardo f. Ven. IV p. 16 ; f. it. del. 471.

ex s: Moug. et Nestler stirp. vog. 375. Rabh. hb. myc. II 48. Fuckel f. rhen. 1049, 1825. Plowright sphaer. brit. I 23. Cooke f brit. II 469. Rehm Ascomyc. 219. Thümen myc. un. 1364.

Asci fusiformes, longe stipitati, pars sporifera $20-24 / 6$, 8 spori. Sporidia oblonga, obtusa, subcurvata, dilute flavidula, 1 cellularia, disticha, 6/2. Paraphyses articulatae, c. 3 mikr. crassae. Ope liq. kali caust. materia flava perithecii solvitur, sed non decoloratur. Jod -

Ad ramulos siccos Pruni Coryli et Betulae prope Malomviz (Hunyad) Transsylvaniae. 8/1873 Lojka.

Edita in Rehm Ascomyc. 219.

10. Eutypa aspera (Nitschke pyren. I p. 132 sub Valsa) Fuckel symb. myc. p. 214.

s yn: Eutypa polycocca var - Karsten myc. fenn. II p. 128. Eutypa aspera Fr. cfr Cooke in Grevillea VI p. 128.

c f r Saccardo f. Ven. IV p. 16.

ex s: Fuckel f. rhen. 1981. Rehm Ascomyc. 218.

Asci fusiformes, longe stipitati, 8 spori, c. $90 / 6$. Sporidia oblonga, obtusa, subcurvata, 1 cellularia, dilute flavidula, disticha in superiore asci parte, c. 7/2,5.

Ad radices Evonymi verrucosi in valle Nuksoraensi infra alpem Retyezát (Hunyad) Transsylvaniae. 1873 Lojka sub 2464.

Videtur ad hanc speciem trahenda!

11. Cryptovalsa ampelina (Nke pyren. I p. 156 sub Valsa). 
c f r Fuckel symb. myc. p. 212. Saccardo myc. Ven. p. 211. syn: Echusias Vitis Hazslinszky Közlem. X 1873 p. 51 tab. I f. $1-2$.

exs: Fuckel f. rhen. 1824 (sub V. protracta). Rehm Ascomye 79 .

Asci fusiformes, longe stipitati, c. $150 / 6-7$, c. 30 spori Sporidia oblonga, obtusa, subcurvata, dilute fla vidula, $7-8 / 2,5$.

Ad sarmenta putrida Vitis viniferae prope Kaposvár (Somogy) 5/1871 Lojka.

Edita in Rehm Ascomyc. 79.

12. Cryptovalsa Pruni Fuckel symb. myc. Nachtrag I p. 321. e f r Saccardo in Michelia I p. 22 (qui syn: Cryptovalsa Rabenhorstii declarat!)

exs: Fuckel f. rhen. 2336.

Asci clavati, longe stipitati, c. 100/7, 80-90 spori. Sporidia oblonga, obtusa, subcurvata, 1 cellularia, $5-6 / 2,5$.

a. Ad ramulum siccum Pruni prope Kaposvár (Somogy) 1872 Lojka sub 146.

b. ibidem prope Malomviz (Hunyad) Transsylvaniae. 8/1873 Lojka. (Sporidia $9 / 2,5$, c. 30 in ascis.)

Specimina haud certa adhuc allata!

13. Valsa (Leucostoma) cincta (Fr. sub Sphaeria) Nitschke pyren. I. p. 228.

m in im e $=$ Valsa cincta Curr. Linn. Trans. XXII. e fr Cooke hdb. p. 817.

c fr Fuckel symb. myc. p. 202. Revue myc. 11 p. 47.

exs: (? Fuckel f. rhen. 2140), Rehm Ascomyc. 224. Speciminulum huc ducendum videtur.

Asci elliptici, c. 40/8-9, 8 spori. Sporidia oblonga, obtusa, 1 cellularia, hyalina, subcurvata, 12/2,5, disticha.

Ad ramum siccum Pruni prope Malomviz Transsylvaniae. Lojka.

14. Valsa (Monosticha) Syringae Nitschke pyren. I p. 189.

ex s:? Thümen myc. un. 1363 (non Ascomycetes in explr meo.)

Speciminulum hue forsitan trahendum!

Asci clavati, 8 spori, 36/5. Sporidia oblongo-elliptica, obtusa, recta vel subcurvata, 1 cellularia, hyalina, disticha, 6/2,5.

Ad ramulos Syringae vulgaris prope Cetati Boli (Hunyad) Transsylvaniae. 8/1873 Lojka sub 2095.

15. Valsa (Monosticha) Orni Rehm nov. spec.

Stroma e basi suborbiculari conico convexum, in cortice in- 
teriore nidulans ejusque materiam non mutans, epidermide circa ostiola facile solubili, pustulata. Perithecia in singulo stromate 8-12, monosticha, dense stipata, globosa, mediocria, in collum attenuata. Ostiola epidermidem vix superantia, in disco minuto aggregata, rotundata, atra, poro pertusa, sessilia. Asci clavati, 8 spori. Sporidia cylindrica, obtusa, vix curvata, 1 cellularia, hyalina 15/3-4.

Ad ramum siccum Fraxini Orni in valle Kolczvárensi (Hunyad) Transsylvaniae. 1873 Lojka.

Secundum speciminulum in herb. meo videtur nova species declaranda!

16. Valsa (Monosticha) decorticans (? Fr. sub Sphaeria) Nitschke pyren. I p. 194.

(n on $=$ Fuckel symb. myc. p. 199, exs. Fuckel f. rhen. $606=\mathrm{V}$. ceratophora f. quercicola.)

exs: bad. crypt. 826 (sub Valsa megas Awd). non Ellis n. am. f. 496 (verisimiliter $=$ ceratophora).

Asci clavati, 8 spori, 906-7. Sporidia oblonga, obtusa, subcurvata, 1 cellularia, disticha, 123.

Ad ramum corticatum Fagi. Kaposvár (Somogy). 1872 sub 153.

Exacte convenit cum exemplaribus badensibus exsiccatis.

17. Valsa (Circinnata) acclinis (Fr. sub Sphaeria).

efr Nitschke pyren. I p. 207.

Asci clavati, substipitati. 8 spori, $-60 /-8$; Sporidia oblonga, obtusa, 1 cellularia, subcurvata, hyalina, disticha, - 11/2,5.

Ad ramulos Pyri Mali. Malomviz (Hunyad) Transsylvaniae. 8/1873 Lojka.

Exemplaria incertae sedis !

18. Valsa (Circinnata) Cypri ('Tul sub Sphaeria) Nitschke pyren. I p. 206.

c fr Saccardo f. Ven. IV 27. Karsten myc. fenn. II p. 142. var. Fraxini.

(f. spermog. Sphaeria pruinosa Fr. efr Cooke hdb. p. 558)

e x s: (Moug. et Nestler stirp. vog. 968, Rabh. f. eur. 1020, Thümen f. austr. 1266, status spermogoniferi!) Rehm Ascomyc. 226.

Asci elliptici, breviter stipitati, - $60 / 12,8$ spori. Sporidia cylindrica, obtusa, recta vel subcurvata, 1 cellularia, hyalina, disticha, -- 21/6. Paraphyses articulatae. Jod -

Ad ramos siccos Fraxini Orni in valle Kolczvárensi infra alpem Retyezát (Hunyad) Transsylvaniae. 8/1873 Lojka.

Edita in Rehm Ascomyc. 226. 
19. Diaporthe (Chorostate) tessera (Fr. syst. sub Sphaeria). Fuckel symb. myc. Nachtrag I p. 318.

efr ? Saccardo f. Ven. nov. V p. 180 (appendiculi sporarum).

ex s: Fuckel f. rhen. 592 (sub Wüstneia). Kunze f. sel. 119 (ubi injuste citatur syn: Rehm Ascomyc. 176). (? Saccardo myc. Ven. 664. Thümen mye. un. 1264).

Asci fusiformes, 8 spori, 66/-9; Sporidia elliptica, subacutata, medio subconstricta, 2-4 cellularia, singulis cellulis 1 nucleo magno praeditis, recta, hyalina, $15 / 3-5$, disticha. Paraphyses filiformes, sittulatae. Jod -

Ad ramulos putridos Coryli. Lunkány in Hung. 7/1872 Lojka sub 1882.

20. Diaporthe (Euporthe) incrustans Nke pyren. I p. 267.

Asci clavati, 8 spori, 5099; Sporidia elliptica, acuta, recta vel navicularia, medio subconstricta, 2 cellularia, utraque cellula nucleis 2 inagnis praedita, hyalina, disticha $10-12 /-4$.

Ad caulem Umbelliferae siccam prope Soborsin in Hung. 7/1872 Lojka sub 1888.

Exemplar incertae adhuc sedis!

21. Diaporthe (Tetrastagon) inaequalis (Curr. sub Sphaeria) Nitschke pyren. I p. 285.

s y n: Sphaeria Fuckelii Duby. Diatrype inaequalis Cooke hdb. p. 813.

ef $r$ Saccardo myc. Ven. p. 140 tab. XIII f. 76-78. Fuckel symb. myc. p. 209.

exs: Fuckel f. rhell. 919. Rabh. f. eur. 2223. Rehm Ascomyc. $276 a, b$.

Asci cylindracei, 8 spori - 90 8. Sporidia biscoctiformia, obtusa, medio subminusve constricta, 2 cellularia, plerumque utraque cellula nucleo 1 magno centrali praedita, hyalina, plerumque 1 sticha 15/--8. Paraphyses filiformes, c. 4 mikr. crassae, guttulatae. Jod -

Ad ramulos emortuos Cytisi hirsuti in sylva Hársas prope Töröcske (Kaposvár, Somogy). 5/1871 Lojka.

Edita in Rehm Ascomyc. $276 b$.

22. Diaporthe (Tetrastagon) Sarothamni Nke pyren. I p. 303. c f r Fuckel symb. myc. p. 207.

ex s: Fuckel f. rhen. 2345. Plowright sphaer. brit. III 31, Thümen myc. un. 1070. Rabh. f. eur. 2428 (n o n Cooke f. brit. I 671). Asci elliptici, 8 spori, 60/7-8. Sporidia elliptica, subacuta, 
plerumque recta, medio subconstricta, 2 cellularia, utraque cellula nucleis 2 instructa, hyalina, disticha, - 18/3,5.

Ad ramulum emortuum Cytisi austriaci prope Szent-Miklós in insula Danubii Csepel (Pest). 6/1873 Lojka sub 1993.

23. Dothidea Sambuci (Pers. sub Sphaeria) Fr. syst.

c f r Fuckel symb. myc. p. 222.

exs: Kunze f. sel. 158. Fuckel f. rhen. 1007. Saccardo myc. Ven. 648 .

Exemplar quoad Hymenium prorsus inevolutum !

Ad ramulum emortuum Sambuci nigrae. Malomviz (Hunyad) Transsylvaniae. 1873 Lojka.

24. Mazzantia Napelli (Ces. sub Dothidea) Passerini, Sacc.

c f r Sacc. f. Ven. IV p. 25.

exs : Rabh. f. eur. 1272,2123.

Asci elliptici, teneri, apice subincrassati, 8 spori, $60-90 / 9-12$. Sporidia elliptica, 1 cellularia, nucleis $2-3$ submagnis praedita, hyalina, disticha, $15 /-5$, saepe una vel utraque apice filiformiter breviter appendiculata. Paraphyses tenerrimae, filiformes, guttulatae, - 4 mikr. crassae. Jod -

An caules putridas Aconiti circa lacum Zenoga infra alpem Retyezát (Hunyad) Transsylvaniae. alt. c. 6000'. 8/1873 Lojka.

25. Thyridium vestitum (Sphaeria - Fr. syst., Valsa - Fr. S. Veg. Tul.) Fuckel symb. myc. p. 195 p. p.

syn: Cucurbitaria vagans Sacc. myc. Ven. p. 122 t. XII f. 34-38, f. ital. 402. Fenestella vestita Sacc. in Michelia I p. 50.

ex s: Fuckel f. rhen. 954. Saccardo myc. Ven. 1272. Rehm Ascomyc. 179 (? Thümen f. austr. 859).

Asci cylindrico-clavati, 8 spori, 150/15. Sporidia subovoidea vel oblonga, obtusa, hyalina, dein fusca, transverse $6-8$ septata, longitudinaliter semel, inde muriformia polyblasta, 1 sticha, 15-18/ 9-10. Paraphyses filiformes, guttulatae, c. 1,5 mikr. crassae. Jod -

Ad ramulos siccos Coluteae arboresc. spontanee crescentis prope Tököl in insula Csepel (Pest). 6/1873 Lojka.

Edita in Rehm Ascomyc. 179!

26. Thyridaria Ailanthi Rehm nov. spec. 1875.

Stromata valsea, conoidea, sub epidermide nidulantia. Perithecia globosa, mediocria, rubrofusca, parenchymatice contexta, c. 8 conglobata, in collum brevissimum attenuata atque ostiolo conoideotruncato, fuscidulo, poroque instructo epidermidem fissam vix superantia. 
Asci cylindraceo-clavati, 8 spori, $75 / 6-7$; Sporidia elliptica, primitus $2-$, denique 4 locularia, ad septa subconstricta, singulis cellulis plerumque nucleo 1 magno praeditis, fuscidula, incurvata, monosticha, $1215 / 3-4$. Paraphyses tenerae, ramosae. Jod -

Ad ramos siccos Ailanthi glandulosae in horto Esterházyano Kaposvár (Somogy). 7/1871 Lojka.

27. Aglaospora profusa (Fr. syst. sub Sphaeria) Tul. De N. s yn: Valsa - Fr. S. V. Sc. (? Massaria Seiridii B. et Br.) efr Cooke hdb. p. 838. Fuckel symb. myc. p. 187. Saccardo myc. Ven. p. 125 tab. XII f. $49-50$, f. it. del. 441.

exs: Moug. et Nestler stirp. vog. 871 p. p. Schweiz. crypt. 622. Rabh. f eur. $733 a, b, 1137,1441$ (sub Cucurbitaria elongata), hb. myc. II 727. Fuckel f. rhen. 583. bad. crypt. 823. Saccardo myc Ven. 650. Thümen myc. un. 969. Rehm Ascomyc. 45. Ellis n. am. f. 172.

Asci cylindraceo-clavati, crassi, 4 spori, 180/24. Sporidia oblonga, obtusa, recta, 4 cellularia, nucleis magnis specifice ordinatis, hyalina, dein fusca, monosticha, - 45/12. Paraphyses ramosae. Jod -

Ad ramos emortuos Robiniae Pseudacaciae. Kaposvár (Somogy). 1872 Lojka.

28. Thyronectria pyrrhochlora (Awd sub Nectria). Saccardo in Michelia III p. 325.

exs: Rabh. f. eur. 1234. Rehm Ascomyc. 40.

Asci clavati, c 80 long., 8 spori. Sporidia oblonga, obtusa, hyalina, transverse 8 septata, longitudihaliter pluries, inde muriformia, polyblasta, - 21/12, 1 - 2 sticha. Paraphyses filiformes. Jod Lojka.

Ad sarmenta Vitis viniferae putrida. Kaposvár (Somogy) 5/1871

\section{Nectria lecanodes Ces.}

? s y n: N. peziza (Tode) Fr. * minor. Desm.

e f $r$ Saccardo in Michelia III p. 290. Fuckel symb. myc. p. 178. Karsten myc. fenn. II p. 215.

exs: Rabh. hb. myc. II 525. luckel f. rhen. 2050. Cooke f. brit. II 564. Rehm Ascomyc. 38. Thümen myc. un. 1746 (? Plowright sphaer. brit. III 12).

Asci cylindracei, 8 spori. Sporidia obtusa, elliptica, medio subconstricta, 2 cellularia, unaquaque cellula 2-4 nucleis magnis praedita, hyalina, monosticha, $-10 /-4$. Paraphyses? Jod -

Parasitica in thallo vivo Peltigerae horizontalis in sylva prope Eperjes. 10/1869 Lojka sub 604. 
30. Nectria episphaerica (Tode sub Sphaeria) Fr. S. V.

c f r Fuckel symb. myc. p. 181. Saccardo in Michelia I p. 291, myc. Ven. p. 123. Cooke hdb. p. 785. Karsten myc. fenn. II p. 124.

exs: Fuckel f. rhen. 981. Thümen myc. un. 766. Ravenel f. am. 340. Plowright sphaer. brit. I 11. Cooke f. brit. I 493. Rabh. f. eur. 642. Rehm Ascomyc. 585. Ellis n. am. f. $469 a, b$.

Asci cylindracei, 8 spori, 45/6. Sporidia ovoidea vel oblonga, obtusa, 2 cellularia, medio interdum subconstricta, interdnm cellulis inaequalibus, monosticha, hyalina, $-8 /-5$. Paraphyses ramosae. Jod -

a. Ad Diatrype Stigma. Malomviz (Hunyad) Transsylvaniae; 8/1873 Lojka.

Exemplaria inevoluta!

b. ad Hypoxylon? prope Kaposvár (Somogy). Lojka sub 71.

c. ad Anthostoma Schmidtii (?). Kaposvár (Somogy). 1871 Lojka sub 197.

31. Nectria minutissima Rehm nov. spec.

Perithecia minutissima, nudo oculo vix conspicua, conoidea, tremelloidea, eburnea, dispersa, in thallo Ophioboli rubelli sessilia. Asci clavati, 8 spori. Sporidia elliptica, subobtusa, medio subconstricta, 2 cellularia, quaque cellula nucleo 1 magno praedita, disticha, hyalina, 12-14/4. Paraphyses? Jod -

Ad caules Umbelliferarum putridas in horto Eszterházyano Kaposvár (Somogy). Lojka 1871.

Species difficillime rite cognoscenda ob minutiem peritheciorum, inde forsitan adhuc dubia!

32. Nectria Cucurbitula (Tode sub Sphaeria) Fr. S. V.

efr Fuckel symb. myc. p. 180. Saccardo in Michelia I p. 290,409 .

exs: Fuckel f. rhen. 983. Schweiz. crypt. 323. Rabh. hb. myc. II 248. f. eur. 1235. Cooke f. brit. I 581. (? Kunze f. sel. 105). Asci cylindracei, 8 spori. Sporidia biscoctiformia, apice obtusa vel subacutata, 2 cellularia, quaque cellula nucleo 1 magno praedita, hyalina, monosticha, $-14 / 6$. Paraphyses filiformes.

Ad Abietum corticem prope Teplicska et Zatracsano infra alpem Dzurowa (Liptó). S/1872 Lojka sub 1358.

33. Nectria chlorella (Fr. El. sub Cenangium) Tul. carp.

c f r Michelia I p. 289 (sporidia 1 septata!), II p. 325.

Asci cylindracei, rarius fusiformes, 8 spori, c. $60 /-7$. Sporidia ovoidea vel elliptica, obtusa 2 cellularia, ad septa haud constricta, 
hyalina, 1 (rarius 2) sticha, $-12 /-5$. Paraphyses filiformes. Perithecium viridiflavum, parenchymaticum. Jod -

Ad ramulos emortuos Abietis. Bandrów in Galicia australi. 8/1869 Lojka sub 6.

34. Nectria cinnabarina (Tode et Fr. sub Sphaeria) Tul.

c f r Fuckel symb. myc. p. 177. Cooke hdb. p. 781. Saccardo in Michelia I p. 289, myc. Ven. p. 122. Karsten myc. fenn. II p. 212.

exs: ? Moug. et Nestler stirp. vog. 570. Rabh. hb. myc. II 633, f eur. 324, 924, 1631. Thümen f. austr. 1050. Fuckel f. rhen. 2657. Cooke f. brit. II 474. Saccardo myc. Ven. 767 (? 926). Ravenel f. am. 339. Plowright sphaer. brit. I 7. Ellis n. am. f. 468. Rehm Ascomyc. 184 (f. obscurata).

Asci cylindraceo-clavati, 8 spori, c. 60 long. Sporidia oblonga, obtusa, recta, medio non constricta, 2 cellularia, hyalina, disticha, $-18 /-5$. Paraphyses filiformes.

a. Ad ramulos siccos. Kaposvár (Somogy) 1871 Lojka sub 185.

b. Ad sarmenta putrida Vitis viniferae. Kaposvár (Somogy) Lojka sub 119.

Sporidia 12/4, itaque forsitan N. viticola B. et C. (cfr Saccardo in Michelia III p. 203).

c. Ad ramulos emortuos Sambuci nigrae. Malomviz (Hunyad) Transsylvaniae 8/1873 Lojka.

Exemplar plane inevolutum itaque dubium!

35. Gibberella pulicaris (Fr. syst. sub Sphaeria). Saccardo in Michelia I p. 43, 317.

syn: Gibbera - Fr. S. V. Sc., Fuckel symb nyc. p. 167. Botryosphaeria - Ces. et De N., Nectria - Tul.

c fr Saccardo myc. Ven. p. 118 tab. XVII f. 10; f. Ven. IV p. 5. Cooke hib. p. 780.

exs: Rabh. hb. myc. II 735, 644 (sub Sphaeria cyanogena Desm.). Fuckel f. rhen. 789. Saccardo myc. Ven. 652, 653 (sub f. baccata Wallr. non = baccata Fuckel symb. myc. p. 306), 1465, 1466. Rehm Ascomyc. $230,489$.

Asci elliptici, tenerrimi, 8 spori, -70,12. Sporidia elliptica, subobtusa, recta vel subcurvata, 4 cellularia, ad septa interdum subconstricta, disticha, hyalina, - 21/5. Paraphyses ramosae? Perithecium parenchymaticum, coeruleum. Jod -

Ad ramulos siccos Sambuci nigrae prope Malomviz (Hunyad) Transsylvaniae. 8/187: Lojka. 
Edita in Rehm Ascomyc. 230.

36. Gibberella pulicaris (Fr.) Saccardo.

var. S a u bineti i Dur. et Montg sub Sphaeria).

s yn: Gibbera - Fuckel symb. myc. p. 168. Botryosphaeria dispersa De N., B. cyanogena (Desm. sub Spaeria), Gibbera flacca (Wallr. sub Sphaeria) Fuckel symb myc. p. 168.

e f r Saccardo myc. Ven. IV 5-6, myc. Ven. p. 117. tab. XII"f. 8-9. Michelia I p. 317, 513. Niessl in Hedwigia 1873 p. 131. exs: Saccardo myc. Ven. 927, 1467, 1468. Ellis n. am. f. 81. Fuckel f. rhen. 976, 2046. Rehm Ascomyc. 381.

Asci fusiformes, teneri, 8 spori, 60/10. Sporidia elliptica, 2 cellularia, hyalina, disticha, 18/4 -6. Paraphyses filiformes. Perithecium parenchymaticum, coeruleum.

Ad caules putridas Zeae Maydis prope Kasposvár (Somogy). 1872 Lojka sub 184.

Exemplar inevolutum, itaque sporidia 2 cellularia!

37. Calonectria dacrymycella (Nyl. sub Sphaeria) Saccardo.

s y n: Nectria - Karsten mye. fenn. II p. 216.

e f $\mathrm{ryl}$. pez. fenn. p. 92, flora 1863 p. 323. Michelia I p. 314, II 163.

ex S: Rehm Ascomyc. $232 a, b$ (sub Nectriella).

Asci fusiformes, 8 spori, $-70 / 11$. Sporidia elliptica, subacutata, 2 cellularia, utraque cellula nucleis $1-2$ magnis praedita, medio interdum subconstricta, recta, hyalina, disticha, -18/-4. Paraphyses ramosae, c. 3 mikr. crassae. Jod -

Ad caules putridas Umbelliferarum prope lacum Zenoga infra alpem Retyezát Transsylvaniae, alt. c. $6000^{\prime}$. 8/1873 Lojka.

Edita in Rehm Ascomyc. $232 a$.

Species distinctissima et in alpibus editissimis Tyrolensibus iterum iterumque a me observata.

38. Calonectria lichenicola (Ces. sub Cryptodiscus) Rehm.

syn : ? Nectriella Robergei Desm. (c f r Nyl. pez. fenn. p. 90). Nectria lichenicola Saccardo in Michelia I p. 289. N. Peltigerae Ph. et Pl. (cfr Grevillea IV p. 223). Nectriella carnea Fuckel symb. myc. p. 176 (? tab. IV f. 19); Nectria erythrinella (Nyl. sub Sphaeria) Tul. I(cfr Karsten myc. fenn. II p. 217. Michelia I. p. 289). ? Nectria Kalchbrenneri Fuckel symb. myc. p. 177. ? N. Peziza Fr. (cfr Arnold in Flora 1881 p. 327, Winter in Flora 1872 p. 523). ex s: Rabh. hb. myc. II 523. Rehm Ascomyc. 37. (? Fuckel. f, rhen. 1835. Plowright sphaer. brit III 13. 
Asci fusiformes vel elliptici, 8 spori, c. 60/15. Sporidia elliptica vel ovoidea, obtusa, 2 cellularia, medio subconstricta, quaque cellula 1-2 nucleata, hyalina, disticha, 15-18/7-8. Paraphyses?

Ad thallum Peltigerae horizontalis in valle Kolczvárensi prope rivulum Riu sor infra alpem Retyezát (Hunyad) Transsylvaniae. 8/1873 Lojka sub 2264.

39. Cucurbitaria pithyophila (Kunze et Fr. sub Sphaeria) De N. schem. p. 214.

c f r Fuckel symb. myc. p. 172 Saccardo f. it. del. 522.

ex s: Rehm Ascomyc. 147 (? Rabh. f. eur. 645, 1337, Fuckel f. rhen. 1814).

Asci cylindracei, apice incrassati, 8 spori, 105/ 8. Sporidia elliptica, subobtusa, recta, hyalina, demum fuscidula, monosticha, 4-8 cellularia, interdum una alterave media cellula semel longitudinaliter divisa, ad septa vix subconstricta, -- 18/5. Paraphyses ramosae. Jod -

Ad ramum siccum Pini. In monte Siodelko prope balneum Szczawnica in Galicia. 1869 Lojka 978.

40. Cucurbitaria elongata (Fr. sub Sphaeria) Grev.

c f r Fuckel symb. myc. p. 174. Cooke hdb. p. 840. Saccardo myc. Ven. p. 120 tab XII f. 25-27. f. it. del. 529.

ex s: Fuckel f. rhen. 970 (? 1815). Moug. et Nestl. stirp. vog. 873 p. p. Rabh. f. eur. 882 . Thümen myc. un. 565 (? f. austr. 252). Rehm Ascomyc. 185, 336 (? Saccardo myc. Ven. 95), non Plowright sphaer. brit. I 54 (Lophiostoma).

Asci cylindracei, 8 spori, 125/10. Sporidia elliptica, recta, transverse 4-6 septata, longitudinaliter plerumque semel, ad septa subconstricta, hyalina, demum fuscidula, monosticha, 18 24/9-10. Paraphyses articulatae, ramosae. Jod -

Ad caules siccas Astragali virgati in terra arenosa dicta "Buczka" in insula Csepel Danubii (Pest). 1873 Lojka.

Edita in Rehm Ascomyc. 185.

41. Cucurbitaria acerina Fuckel symb. myc. p. 172 tab. IV f. 3. ex s: Fuckel f. rhen. 2255 (explr meum nimis mancum!)

Asci cylindracei, 8 spori. Sporidia oblonga, obtusa, transverse in cellulas 6 septata, singula semel longitudinaliter, medio subconstricta, hyalina, dein fuscidula, monosticha, 18/7-8. Paraphyses filiformes. Jod -

Ad ramulos siccos Aceris camp. in horto Esterházyano. Kaposvár (Somogy). 10/1871 Lojka sub 2533. 
42. Staurosphaeria Lycii (Duby sub Dothidea) Rabh.

s yn: St. varians (Hazslinszky sub Cucurbitaria) NiessI.

ex s: Zopf et Sydow myc. march. 82. Rehm Ascomyc. 593. Rabh. t. eur. 55, 1537 (hb. myc. II 736. Kunze f. sel. 261).

Perithecia sparsa, rotunda, atra, c. $1 \mathrm{~mm}$. diam., sub peridermio pustulatim elevato nidulantia, peridermio vario modo dilacerato et ostiolo, primitus albido, dein denigrato, perspicue late pertuso, ostiolo dein prorumpente. Stratum corticis interioris longe lateque nigrificatum. Perithecia parenchymatica, fusca. Asci cylindracei, 8 spori, 200/15. Sporidia elliptica, subacuminata, primitus 4 cellularia, dein longitudinaliter utraque cellula media septata, denique 6-12 cellularia, ad septa subconstricta, fuscidula, 15-17/ 9 -10. Paraphyses crassae, ramosae. Jod -

Ad ramulos siccos Lycii barbari in horto Esterházyano Kaposvár (Somogy). 5/1871 leg. Lojka.

Exemplaria sporis paullulum minoribus a forma normali divergunt!

43. Bertia moriformis (Tode sub Sphaeria) Fr. S. V. et De N

c f r Fuckel symb. myc. p 164. Cooke hdb. p. 861. Karsten myc. fenn. II p 160. Saccardo f. it. del. 440.

ex s: Fuckel f. rhen. 999. Moug. et Nestler stirp. vog. 382. Cooke f. brit. I 586, II 487. Plowright sphaer. brit. 67. Rehm Ascomyc. 442 (? Rabh. hb. myc. II 637).

Asci clavati, in stipitem brevem angustati, 8 spori, $120 / 18$ Sporidia dactyloidea, obtusa, recta vel subcurvata, 1 (? 2) cellularia, nucleis $2-4$ inagnis praedita, hyalina, tristicha, - 36/4 8. Paraphyses articulatae, crassae. Jod -

a. Ad truncos Abietum putridos in valle Riu sor infra alpem Retyezát Transsylvaniae. 8/1872 Lojka sub 1875.

b. Ad ramum Pini decorticatum in regione Turkului infra alpem Retyezát (Hunyad) Transsylvaniae. 7/1872 Lojka sub 1691.

44. Bertia lichenicola De N. in erb. critt. it. no 1190 (1864!)

s y 11: Rhagadostoma corrugatum Koerb. par. p. 473. Psilosphaeria - Cooke in Grevillea VIII p. 85 (? Sphaeria - Cooke in Grevillea I p. 156).

c f r Anzi anal. lich. p. 26. Arnold fragm. lich. XVI in Flora 1874. Saccardo f. it del. 439. (asci 4 spori !)

ex s: Rabh. f. eur. 950. Rehm Ascom. 283.

Asci elliptici, stipitati, 2 spori, 90/15. Sporidia dactyloidea, obtusa, 1 - 2 cellularia vel 2-4 nucleis magnis instructa, recta vel subcurvata, hyalina, parallela, 40-42/6-8. Paraphyses? Jod - 
a. Parasitica ad thallum Solorinae croceae. In jugo 'Tatrae, Kopa dicto. 8/1868 Lojka sub 162.

b. ibidem in cacumine alpis Retyezát (Hunyad) Transsylvaniae, alt. 2477 m. 1874 Lojka sub 2999.

45. Trematosphaeria (Winteria) lichenoides Rehm nov. spec. 1875.

syn: Zignoella - Sacc. in Michelia I p. 347.

ex s: Rehm Ascomyc. 285.

Perithecia - 0,5 $\mathrm{mm}$ diam., nigroviridula, haud carbonacea, dimidiata, semiglobosa, umbilicato-pertusa, in ligni dealbati superficie insidentia, denique foveolam nigrolimitatam relinquentia, sparsa. Asci subclavati, 6-8 spori, 90/-11. Sporidia obtuse elliptica, 2-4-6 cellularia, quaque cellula $1-$ pluribus magnis nucleis praedita, ad septa haud constricta, $1-1 \frac{1}{2}$ sticha, hyalina, $-20 /-8$. Paraphyses filiformes. Jodii ope Hymenium valde coerulescit.

Ad truncum Pini decorticatum circa lacum Zenoga infra alpem Retyezát (Hunyad) Transsylvaniae. Lojka sub 2410.

(Winteria Rehm nov. subgenus Trematosphaeriae. Perithecia haud carbonacea, sed viridi - vel fusce parenchymatica, collabentia, coriacea, non apiculata, sed patellaeformia, ostiolo pertusa. Hymenium Jodii ope plerumque coerulescens).

46. Zignoella transsylvanica Rehm nov. spec.

Perithecia solitaria, in ramorum superficie insidentia, ita ut globosa, integra, c. $0,8 \mathrm{~mm}$ diam. immersa sint, sed pars conica c. $0,5 \mathrm{~mm}$ longa exstet, nigerrima, poro pertusa, fragilia. Asci cylindracei, apice incrassati, 8 spori, 250/9. Sporidia elliptica, subacuminata, hyalina, 20-24 locularia, monosticha, 45-50/-9. Paraphyses filiformes. Jod -

Ad ramum siccum Syringae vulgaris. "Slima Leorda" prope Petrila (Hunyad) Transsylvaniae. 1873 Lojka sub 2085.

Species valde insignis!

47. Trematosphaeria Morthieri Fuckel symb. myc. Nachtrag I p. 306.

s y n : Zignoella - Saccardo in Michelia I p. 347. Trematosphaeria picastra Fuckel symb. myc. p. 162.

exs: Fuckel f. rhen. 2447. Thümen myc. un. 167. Rehm Ascomyc. 87.

Perithecia dimidiata; Asci clavati, 8 spori, - 66/15. Sporidia cuneiformia, obtusa, recta, 4 cellularia, 18-21/6, hyalina, disticha. Paraphyses filiformes. Jod -- 
1870 Lojka.

Ad truncum decorticatum Pini in sylva Kislakensi (Somogy).

48. Melanomma pertusum (Pers. sub Sphaeria) Rehm.

s y n : Trematosphaeria - Fuckel symb. myc. p. 162.

c f r Karsten myc. fenn. II p. 90. Cooke hdb. p. 869. Michelia I p. 345.

ex s: Plowright sphaer. brit. III 50. Saccardo myc. Ven. 691.

Asci clavati, 8 spori, 75/10. Sporidia elliptica, recta vel subcurvata, 2 cellularia, medio subconstricta, dein 4 cellularia, quaque cellula nucleo 1 magno instructa, fuscidula, disticha, $18-24 /-6$. Paraphyses filiformes. Jod sub 88 .

Ad ramum decorticatum. Kaposvál (Somogy). 1872 Lojka

Nescio an revera exemplar ad speciem nominatam, valde dubiam pertineat! Sed cum allatis exs. congruit.

49. Melanomma Catillus Sace. myc. Ven. p. 114. tab. XI. f. $39-42$.

c f r Michelia I p. 344. Saccardo f. it. del. 466.

Asci cylindracei, 8 spori. Sporidia elliptica, obtusa, 4 cellularia, ad septa subconstricta, nucleolis praedita, fuscidula, monosticha, 15/-5. Paraphyses ramosae?. Jod -

Verisimiliter ad Winteriam trahenda species!

Ad lignum querneum putridum in valle Bilian infra montem Domugled prope thermas Herculis (Szörény) in Hungaria. 1874 Lojka sub 2756.

50. Melanomma Aspegrenii (Fr. sub Sphaeria) Fuckel symb. myc. p. 159 tab. III f. 29.

Asci cylindracei, 8 spori. Sporidia elliptica, subacutata, medio constricta, 2-4 cellularia, quaque cellula nucleo 1 magno praedita, fuscidula, monosticha, $-15 / 6$. Paraphyses filiformes. Jod -

Ad ramulos putridos. Kaposvár (Somogy) 1872 Lojka sub 177

Mihi valde dubium, an exemplaria ad speciem nominatam pertineant!

51. Amphisphaeria pinicola Rehm 1872!

s y n : Didymosphaeria alpina Hazslinszky in Közlem. 1873 p. 49.

ex s: Rehm Ascomyc. 135 (sub Amphisphaeria betulina Lahm).

Perithecia erumpentia, dein in cortice sessilia, semiglobosa, breviter papillata poroque pertusa, dimidiata, atra, sparsa, c. $0,5 \mathrm{~mm}$ diam. Asci cylindracei, (4-) 8 spori, 120/15. Sporidia elliptica, primitus hyalina et 1 cellularia, dein medio constricta, 2 cellularia, 
fusca, massa granulosa repleta, 1-2 sticha, 21-24/-10. Paraphyses ramosae, numerosissimae. Jodii ope Hymenium coerulescit.

Ad ramulos Pini Mughi in monte „Schusterknöppchen" Tátra in Hung. 1873 Lojka sub 278.

52. Lophiostoma (Schizostoma) vícinellum Sacc. in Michelia III p. 335,337 ; f. it. del. 240.

Asci clavati, S spori, 75/12. Sporidia fusiformia, subobtusa, medio constricta, 2 cellularia, quaque cellula nucleis 2 magnis praedita, fuscidula, disticha, $216-7$. Paraphyses filiformes. Jol -

Ad ramulos siccos Artemisiae camp. in insula Csepel Danirbii (Pest). 1873 Lojka sub 1992.

53. Lophiostoma quadrinucleatum Karsten myc. fenn. II p. 85.

c f r Saccardo f. Ven. III p. 4, f. it. del. 222, 237. Grevillea VIII p. 107.

Asci clavati, 8 spori, 90/-10. Sporidia oblonga (- elliptica), obtusa, 4 cellularia, ad septa subconstricta, quaque cellula nucleo 1 mediocri praedita, $-17,6$, disticha. Paraphyses tenerrimae, filiformes. Jod -

a. Ad ranum siccum Rosae in valle Kolczvárensi infra alpem Retyezát Transsylvaniae. 8/1873 Lojka.

b. Ad ramum siccum Pruni spinosae prope Malomviz Transsylvaniae. 8/1873 Lojka.

54. Lophiostoma perversum De N.

c f r Sacc. f. Ven. III p. 4 ; f. it. del. 228. Michelia III $p$ 333,339 (sporidia appendiculata!)

Asci clavati, 8 spori, $-130 / 15$. Sporidia fusiformia, acutata, recta vel subcurvata, 6 cellularia, saepe ad septa subconstricta, primitus quaque cellula nucleo 1 magno instructa, haud appendiculata, fuscidula, disticha, - 36/6. Paraphyses filiformes. Jod -

Ad lignum quercinum. Kaposvár (Somogy). 1872 Lojka sub 165.

55. Lophiostoma macrostomum ('Tode sub Sphaeria) Fuckel symb. myc. p. 157. I p. 334 .

syn: L. pseudomacrostomum Sacc. f. it. del. 234. Michelia

ex s: Saccardo myc. Ven. 679. Rehm Ascomyc. 529.

Asci clavati, 8 spori, 105/9. Sporidia elliptica, subobtusa, 6-8 cellularia, ad septa vix constricta, recta, hinc inde una alterave cellula media semel longitudinaliter divisa, $1-1 \frac{1 / 2}{2}$ sticha, fusca, 15-20/5. Paraphyses filiformes. Jod -

Ad ramum decorticatum prope Bandrów in Galicia. 1869 Lojka 
sub 811. Mihi dubium videtur, an exemplar ad hanc speciem pertineat, nempe sporidiis diversum ab Rehm Ascomyc. 529.

56. Lophiostoma macrostomoídes De N.

c fr Sacc. in Michelia I p. 334, 339, f. it. del. 236.

exs: Rehm Ascomyc. 482.

(Asci clavati, 8 spori, 175/24. Sporidia elliptica, obtusa, $8-10$ cellularia, cellula tertia ampliori ceteris, quaque cellula nucleo magno 1-2 centrali praedita, hyalina, demum fusca, massa gelatinosa tenui involuta, disticha, 30-36/10. Paraphyses ramosae. Jodii ope sporidia adulta coerulescunt).

Ad ramulos siccos Artemisiae camp. in insula Danubii Csepel (Pest). 1873 Lojka sub 1992.

Non congruit cum speciminibus in Rehm Ascom. sub hoc nomine editis. Itaque incertum, an recte sit denominata!

57. Lophiostoma insidiosum (Desm. sub Sphaeria) Ces. et De N.

s y n: Lophiostoma appendiculatum Niessl, simillimum Karsten myc. fenn. II p. 84, caulium Fuckel symb. myc. p. 156, Nachtrag III p. 20.

c f r Winter in Flora 1872 p. 543. Saccardo myc. Ven. III p. 23 f. 11, f. it. del. 226, 227. Michelia I p. 340, 447. Iledwigia 1875 p. 70.

ex s: Rabh. f. eur. 1871. Rehm Ascomyc. 88. Fuckel f. rhen. 927. Rabh. f. eur. 1725 (sub Leptosphaeria Artemisiae). Zopf et Sydow myc. march. 67.

Asci clavati, 8 spori, - $-90 / 14$. Sporidia elliptica, obtusa, recta vel subcurvata, 6 cellularia, plerumque tertia cellula latiori, medio plus minusve constricta, subconstricta saepe ad cetera septa, saepe (juaque cellula $1-2$ nucleis praedita, utraque apice hyalina, filiformi, c. ${ }^{1 / 3}$ long. sporidii appendice instructa, fuscidula, disticha, $-216-7$. Paraphyses filiformes. Jodii ope sporidia adulta dilute coerulescunt.

1. Ad caules emortuas Artemisiae camp. et aliarum Compositarum in insula ('sepel Danubii (Pest). 1873 Lojka sub 1992.

b. Ad caules putridas Umbelliferarum. Kaposvár (Somogy) 1871 Lojka.

58. Lophiostoma caulium (Fr. sub Sphaeria) De N.

e f $r$ Cooke hdb. p. 851. Niessl in Hedivigia 1875 p. $21-24$. Vichelia I p. 339. Karsten myc. fenn. I p. 87.

exs: Rehm Ascomyc. 181, 484 (f. minuta).

Asci clavati, 8 spori, 105 12. Sporidia elliptica, plerumque 
subobtusa, recta, 6 cellularia, 15/6, hyalina, dein fuscidula, disticha, $15 / 5$ - 6. Paraphyses filiformes. Jod -

a. Ad ramulos siccos Ononidis spin. Szent-Miklós in insula Danubii Csepel (Pest'. 1873 Lojka sub 2001.

b. Ad caules putridas Zeae Maydis prope Kaposvár (Somogy) 6/1871 Lojka.

Differt imprimis sporidiis $18-20 / 4$, itaque forsitan ad aliam speciem trahenda!

59. Lophiostoma (Lophiotrema) glaciale Rehm nov. spec. 1879!

(? syn. Lophiotrema affine Spegazzini; cfr Saccardo f. it. del. 610$)$.

exs: Rehm Ascomyc. 183 (sub ? L. Sedi Fuckel).

Perithecia c. $0,0 \mathrm{~mm}$ diam., primitus gregarie sub epidermide denigrata nidulantia, dein superficialia, dimidiata, hemiglobosa, in papillam pectinatam, angustam, elongatam, protracta, atra, denique foveolam albescentem, rotundam relinquentia. Asci clavato-pedicellati, apice incrassati, 8 spori, -- 120/10. Sporidia elongato-fusiformia, subcurvata, bicellularia, medio constricta, quaque cellula nucleo 1 magno praedita, hyalina, $-2 \mathbf{2} / 5$, utraque apice appendice setif rmi, 5-6 mikr. long., hyalina instructa, disticha. Paraphyses filiformes. Jod -

Ad caules putridas Aconiti in alpe Dzurova prope Teplicska (Liptó) alt. c. 5000'. 8/1872 Lojka sub 1389.

Edita in Rehm Ascomyc. 183 !

60. Lophiostoma (Lophiotrema) crenatum (Pers. sub Sphaeria) Sace. f. it. del. 359.

c f r Michelia IV p. 412 (? Karsten myc. fenn. II p. 86). exs: ? Fuckel f. rhen. 1808.

Asci clavati, 8 spori, c. 100/12. Sporidia elliptica, recta, medio interdum subconstricta, 2 cellularia, utraque cellula nucleis 2 magnis instricta, dein $4-6$ cellularia, hyalin a, disticha, 27/5-7. Paraphyses filiformes. Jod --

a. Ad ramulos Artemisiae camp. siccos in insula Csepel (Pest) Danubii. 1873 Lojka sub 1992.

b. A ramulos decorticatos ? Csetátye Bóli (Hunyad) Transsylvaniae 1873 Lojka sub 2093.

61. Lophiostoma (Lophiotrema) ampelinum Rehm nov. spec. 1873.

Perithecia $0,3 \mathrm{~mm}$ diam., gregaria, globosa, fere plane cortici immersa, vix protuberantia nisi ostiolo pectinato, crasso, brevi, nigra, 
corticis superficie denigrata. Asci cylindraceo-clavati, crassi, 8 spori, -90/8; Sporidia elongato-fusoidea, subacuta, recta, 4-8 cellularia, ad septa haud constricta, hyalina, disticha, $-27 / 4$. Paraphyses ramosae, tenerrimae. Jod -

Ad corticem trunci Vitis vinif. spontanee crescentis in sylva Kislakensi prope Kaposvár (Somogy). 7/1871 Lojka sub 123.

62. Lophiostoma (Lophidium) compressum (Pers. sub (Sphaeria). Ces. et De N.

sy n: Mytilostoma - Karsten symb. VI p. 36 (? M. deflectens et subcompressum ibid). Lophiostoma angustatum Fuckel symb. myc. p. 158. Nachtrag I p. 16. Saccardo myc. Ven. 210 , f. it. del. 233. Michelia I p. 334, 3:0 Karsten myc. fenn. II p. 83. Grevillea VIII p. 107.

e x s : Fuckel f. rhen. 924, 925. Rabh. f. eur. 340 (sub Cucurbitaria elongata). Cooke f. brit. I 661 (sub L. bicuspidata). Rehm Ascomyc. $182 a, b$. Thümen myc. un. 1548, 1457. Kúnze f. sel. 341. Saccardo myc. Ven. 1476.

Asci clavati, 8 spori, c. $100 / 15$. Sporidia elliptica, obtusa, recta, 4-6 cellularia, cellulae mediae plerumque semel longitudinaliter divisae, ad septa subconstricta, fuscidula, disticha, - 18/7. Paraphyses ramosae. Jod -

Ad caules siccas ? Kaposvár (Somogy). 1873 Lojka sub 1995.

63. Didymosphaeria conoidea Niessl neue Kernpilze.

c f r Hedwigia 1875 p. 152. Saccardo f. Ven. II p. 314 obs. II, f. it. del. 208.

exs: Kunze f. sel. 326.

Asci cylindraceo-clavati, $-70 / 6,8$ spori. Sporidia dacryoidea, obtusa, 2 cellularia, fusca, monosticha, $8 / 5$. Paraphyses filiformes. Jod -

Ad caules putridas Aconiti Napelli infra alpem Retyezát (Hunyad) Transsylvaniae alt. c. $6000^{\prime}$. 8/1872 Lojka.

64. Rebentischia unicaudata (B. et Br. sub Spaeria).

c f r Cooke hdb. p. 892. Saccardo consp. p. 12.

exs: Rabh. f. eur. 50. Rehm Ascomyc. 241.

Asci clavati, crassi, 8 spori, 60/15. Sporidia oblonga, obtusa, 4 cellularia, ad septa haud constricta, quaque cellula nucleo 1 magno praedita, -21/6; apice una appendiculo hyalino, setiformi, 2 cellulari, c. 6 mikr. long. instructa, fuscidula, disticha. Paraphyses ramosae. Jod -

Ad corticem Clematidis Vitalbae in valle "Riu mare" infra alpem Retyezát (Hunyad) Transsylvaniae. 8/1873 Lojka. 
Edita in Rehm Ascomyc.:

65. Pleospora vulgaris Niessl Notizen p. 27.

c f r Saccardo in Michelia I p. 48, f. it. del. 549.

ex s: Rabh. f. eur. 451, 824, 1332. Saccardo myc. Ven. 1349 (sub Pl. media).

Exemplar mancum sporidiis transverse $4---8$ cellularibus, nonnulis cellulis semel longitudinaliter divisis, $-24 / 7$ huc pertinere videtur.

Ad caules Compositae siccas. Kaposvár (Somogy) Lojka sub 195.

66. Pleospora coronata Niessl Notizen p. 16 tab. IV f. 2.

ex s: Rehm Ascomyc. 591.

Asci crassi, clavati, (4-) 8 spori, $-105 / 15$. Sporidia ovoideoelongata vel elliptica, recta vel subcurvata, in $2-8$ cellulas transverse septata, cellulis mediis plerumque semel longitudinaliter divisis, mellea, disticha, $-30 / 8$; senilia saepe una apice breviter appendiculata. Paraphyses ramosae, guttulatae. Ad basim perithecii hyphae nonnullae fuscae, breves. Jodii ope sporidia coerulescunt.

a. Ad caules siccas Centaureae Tauscheri in terra arenosa „Buczka" prope Szent-Miklós in Insula Danubii Csepel (Pest). 6/1873 Lojka sub 1991.

b. ad caules siccas Artemisiae camp. ibidem. 1873 Lojka sub 1992.

c. ibidem 1873 sub 1995.

d. ad caules putridas Aconiti in alpe Retyezát (Hunyad) c. $6000^{\prime}$ alt. Transsylvaniae. 8/1873 Lojka. Sporidia transverse 10 cellularia. Mihi dubium, an speciminulum meum huc trahendum sit!

67. Leptosphaeria scotophila Sacc. f. Ven. II p. 317.

c f r Michelia I p. 342. Sacc. f. it. del. 282.

Asci cylindraceo-clavati, apice incrassati, 8 spori. Sporidia elliptica, 4 cellularia, ad septa subconstricta, fere hyalina, plerumque monosticha, 21/-7. Paraphyses ramosae. Jod -

Ad caulem siccam Umbelliferae. Lipócz (Sáros) Lojka sub 640.

Speciminulum ad hanc speciem pertinere videtur!

68. Leptosphaeria rubicunda Rehm nov. spec.

c f r Winter in Flora 1872 p. 543 . Saccardo f. Ven. II p. 315, f. it. del. 292.

ex : Rehm Ascomyc. 92.

Perithecia gregaria, in maculis rubris epidermidis immersa, minuta, globosa, in collum brevem attenuata, atra. Asci clavati, 8 spori, 60,9. Sporidia fusoidea, recta vel subcurvata, 4 cellularia, 
cellula secunda plerumque latiori, ad septa subconstricta, dilute flavidula, 2 -- 3 sticha, - 27/3. Paraphyses ramosae, artic ulatae. Jod -

Ad caules putridas Conii maculati in horto Eszterházyano. Kaposvár (Somogy) 1871 Lojka.

Edita in Rehm Ascomyc.

69. Leptosphaeria purpurea Rehm nov. spec.

Perithecia gregaria, primitus epidermide tecta, dein ea secedente libera, in ligni longe lateque purpuree tincti superficie insidentia, hemisphaerica, integra, papilla minutissima pertusa, glabra, atra, c. $0,3 \mathrm{~mm}$ diam., hyphis creberrimis, longiusculis, fuscis, c. 0,3 mikr. diam. basi insidentibus. Asci clavati, 8 spori, 70/8. Sporidia fusoideo-elongata, plerumque recta, 4 cellularia, cellula secunda multo latiori, cellulis nucleo 1 magno praeditis, mellea, 21/3-3,5, disticha. Paraphyses ramosae. Jod -

Ad caules siccas Artemisiae vulgaris in horto Eszterházyano. Kaposvár (Somogy). 1871 Lojka.

70. Leptosphaeria obesa (Dur, et Montagne sub Sphaeria) Saccardo in Michelia I p. 38.

syn: Heptameria elegans Thümen $18^{-} 9$ in herb. meo.

c f r Saccardo f. it. del. 281 .

Asci clavati, 8 spori, $75 / 15$. Sporidia fusoidea, recta vel subcurvata, acutata, cellula media magna, c. 9 mikr. lg., nucleo 1 magno centrali praedita, latiore caeteris cellulis cujusque lateris 4, ita ut sporidia 9 cellularia sint, cellula media fusca, lateralibus fuscidulis, 42/5, disticha. Paraphyses ramosae. Jod -

Ad caules siccas Artemisiae camp. in horto Eszterházyano Kaposvár (Somogy). 7!1871 Lojka.

Species, quoad sporidia egregia!

71. Leptosphaeria modesta (Desm.) Awd.

s y n : L. Cibostii Ces. et Ie N., setosa Niessl, Passerinii Sacc.

cfr Karsten myc. fenn. II p. 106. Cooke hdb. p. 905 (sub Sphaeria). Saccardo in Michelia I p. 37, 343, 511, 49\%, f. it. del. 521, 291 (Passerini).

exs: Kunze f., sel. 74, 257 (๖). Rabh. f. eur. 948. Saccardo myc. Ven. 1352. Fuckel f. rhen. 2439 (sub Pleospora Phyteumatis symb. myc. Nachtrag II p. 25). Thümen f. austr. 481, 1253, ? 483, myc. un. ? 165 (non Fuckel f. rhen. 856).

f. A coniti.

Sporidia fusiformia, obtusa, recta vel subcurvata, 5 cellularia, cellula secunda ceteris latiori, flavidula, $-30,5,2-3$ sticha in 
ascis clavatis $90 / 14$ Paraphyses ramosae. Jodii ope sporidia coerulescunt.

Ad caules siccas Aconiti Napelli in alpe Retyezát, alt. c 6000'. (Hunyad) Transsylvaniae. 8/1872 leg. Lojka.

72. Leptosphaeria macrospora (Fuckel symb. myc. p. 138 sub Pleospora) Thümen.

s y n: L. pyrenopezizoides Speg. et Sacc. cfr Sace. f. it. del. 323.

exs: Thümen myc. un. 1359. Sacc. myc. Ven. 1475.

Asci clavati, 8 spori, 105/12. Sporidia oblonga, obtusa, subcurvata, 4 cellularia, cellula secunda ceteris latiore, flavidula, $-30 / 5$. Paraphyses filiformes.

Ad caules Umbelliferarum putridas prope Kaposvár (Somogy). Lojka.

Videtur haud diversa a. L. Nitschkei Rehm Ascomyc.

73. Leptosphaeria Doliolum (Pers. sub Sphaeria) Ces. et De N.

s y n: Pleospora-Fuckel symb. myc. p. 135.

c f $r$ Cooke hdb. p. 902. Saccardo myc. Ven. p. 103; f. Ven. II 314 , f. it. del. 499. Karsten myc. fenn. II p. 97. Michelia I p. 318. Revue myc. 11 p. 44.

exs: Rehm Ascomyc. 93 a, b. Saccardo myc. Ven. 1350, 1472. Moug. et Nestler stirp. vog. 571. Plowright sphaer. brit. II 79. Cooke f. brit. II 495. Ellis n. am. f. 197. (? Fuckel f. rhen. 901. Rabh. hb. myc. II 545. bad. crypt. 635. Cooke f. brit. I 489. Thümen f. austr. 1255). no n Rabh. f. eur. 1546 (Ophiobolus).

Asci cylindracei, crassi, 8 spori, $-130 / 6$. Sporidia fusoideo . elliptica, (4 raro 6-) cellularia, recta vel subcurvata; cellula media plerumque latiore, saepe quaque cellula nucleo 1 magno praedita, interdum ad septa subconstricta, mellea, $1{ }^{1 / 2}$ sticha, $-25 / 5$. Paraphyses ramosae. Jod -

a. Ad caules siccas Urticae dioicae in horto Eszterházyano. Kaposvál (Somogy) 7/1871 Lojka.

Edita in Rehm Ascomyc. 93 ".

b. ibidem ad caules putridas Umbelliferarum 8/1871 Lojka. Edita in Rehm Ascomyc. $93 b$.

c. ibidem ad caules siccas Daturae Stramonii. 1871 Lojka sub $117 a$.

d. ibidem ad caulem siccam. 1872 Lojka sub 81 .

f. s y nd ol i o la Rehm.

Sporidia 4 (rarissime 6) cellularia, 18-21/3,5-5. Perithecia minuta, extus non rugosa. 
a. ad caule's putridas Conii in horto Eszterházyano. Kaposvár (Somogy) 1871 Lojka sub 40.

Forsitan minutie peritheciorum et sporidiorum revera diversa species!

b. ad caules siccas Peucedani longifolii prope "Kreuz" supra Thermas Herculis in Hung. 9/1872 Lojka sub 2008.

(extus formae normali similis!)

f. c o n o id ea De N.

cf $r$ Cooke hdb. p. 902. Saccardo f. Ven. nov. II p. 314, f. 7!. del. 500. Karsten myc. fenn. II 1. 98.

exs: Rehm Ascomyc. 194, 343. Saccardo myc. Vell. 76. Kunze f. sel. $335,336$.

Asci cylindracei, apice incrassati, 8 spori, $-90,6$. Sporidia fusoideo-elliptica, recta vel subcurvata, saepe altera cellula media latiori, 4 cellularia, quaque cellula plerumque nucleo magno 1 centrali instructa, mellea, ad septa subconstricta, $1 \frac{1 / 2}{2}$ sticha, $-18 / 4$ Paraphyses ramosae. Jod -

a. Ad caules siccas Dipsaci sylvestris prope Lunkany in Hung. 7/1872 Lojka.

Edita in Rehm Ascomyc. 194!

b. Ad caules siccas Angelicae sylv. in monte Retyezít (IIunyad) Transsylvaniae. 8/1873 Lojka.

74. Leptosphaeria Coniothyrium (Fuckel symb. myc. p. 115. Nachtrag II p. 89 sub Sphaeria). Saccardo f. Ven. II p. 317, V p.'200. cfr. Saccardo myc. Ven. 98 tab. IX f. $47-49$, f. it. del. 517.

exs: Rehm Ascomyc. 388. Fuckel f. rhen. 2521 (? 910 sub Sphaeria clypeiformis).

Asci clavati, 8 spori, $-66 / 7$. Sporidia fusoidea vel elliptica, recta vel subcurvata, 4 cellularia, ad septa subconstricta, fuscidula, 10/3, 11/2 sticha. Paraphyses filiformes. Jod -

Ad sarmenta sicca Rubi Jdaei infra Theu ursului prope Lun kány in Hung. 7/1872 Lojka sub 1883.

75. Leptosphaeria agnita (Desm. sub Sphaeria) Ces. et De N. s y n : Pleospora-Fuckel symb. myc. p. 135.

c f r Cooke hdb p. 903. Sacc. f. Ven. nov. II 316, f. it. del. 506. Winter in Flora 1872 p. 523.

ex s: Rabh. f. eur. 826, 1254, 2042, 1138 (sub Pleospora herbarum). Cooke f. brit. II 255. Thümen myc. un. 1257, f. austr. 861 (sub Rhaphidophora Betonicae). Rehm Ascomyc. 35, 144. (? Fuckel f. rhen. 888). 
Asci clavati, \& spori, 90/10. Sporidia dactyluidea, prope me. dium constricta, 7 cellularia, plerumque quaque cellula nucleo 1 magno praedita, recta vel subcurvata, obtusa, flavidula, disticha. 36/5. Paraphyses ramosae. Jod -

Ad caules siccas Centaureae Tauscheri (Kerner) in terra arenosa "Buczka" prope Szent-Miklós insulae Danubii Csepel (Pest). 6/1873 Lojka sub 1991.

76. Massaria polymorpha Rehm nov. spec. 1880.

Perithecia minuta, globosa, c. $0,5 \mathrm{~mm}$ diam., sub epidermide pro parte infuscata in cortice interiore nidulantia eanque pustulatim gregarie elevantia et radiatim findentia, atra, ostiolo minuto pertusa. Asci clavati, apice incrassati, 8 spori, -1!0/12. Sporidia obtuse elliptica, medio subconstricta, 2 cellularia, utraque cellula nucleis 2 magnis praedita, massa gelatinosa lata circumdata, hyalina, - 21/5, 1-1 $\frac{1}{2}$ sticha. Paraphyses ranosae. Jod -

exs: Rehm Ascomyc. 242 (sub Sphaeria intermixta).

Ad ramos siccos Rosarum infra alpem Retyezát (Hunyad) Transsylvaniae. Lojka.

Edita in Rehm Ascomyc. 242!

77. Massaria inquinans (Tode sub Sphaeria) Fr. S. Veg.

c f r Karsten myc. fenn. II p. 82. Saccardo myc. Ven p. 109 tab. $\mathrm{X}$ f. it. del. 413, 414. Fuckel symb. myc. p. 153. Cooke hdb. p. 846 .

ex s: Fuckel f. rhen. 803. Rabh. f. eur. 1237, 1526. Saccardo myc. Ven. 82, 1189 (? Plowright sphaer. brit. II 48).

Exemplaria senilia, sine ascis, sporidiis specifice tetrablastis, fuscis, $57-60 / 15$.

Ad ramulos siccos Aceris camp. Kaposvár. Lojka 1872 sub 154.

78. Rosselinia minima Fuckel symb. myc. p. 149.

Asci cylindracei, 8 spori, 60/5. Sporidia elliptica, 1 cellularia, nucleo 1 centrali magno praedita, fusca, monosticha, 9/4. Paraphyses? Jod -

Ad sarmenta putrida Sedi Telephii prope Kaposvár (Somogy). 1871 Lojka sub 143.

Dubitandum, an exemplaria revera ad hane speciem pertineant!

79. Rosselinia Aquila (Fr. sub Sphaeria) Tul. De N.

s yn: Sphaeria byssiseda Engl. fl.

ef r Fuckel symb. myc. p. 148. Saceardo myc. Ven. p. 108. f. Ven. II p. 32, f. it. del. 586. Michelia I p. 26. Cooke hdb. p. 853 . 
e x s: Fuckel f. rhen. 963,964, 1061 (sub Hypoxylon globulariforme). Rabh. hb. myc. II 648, f. eur. 1016. Noug. et Nestler stirp. vog. 965. Cooke f. brit. II 486. Plowright sphaer. brit. I 61.

= Ravenel f. amer. 365. Ellis n. am. f. 181. (? Sacc. myc. Ven. 1366) n o n Sacc. myc. Ven. 1488.

Asci cylindracei, 8 spori, 135/-7. Sporidia elliptica, 1 cellularia, nucleis 1-.2 magnis instructa et massa gelatinosa tenui involuta, fusca, monosticha, 15-18/5. Paraphyses articulatae, guttulatae. Jodii ope apex ascorum valde coerulescit.

a. Ad ramum putridum. Kaposvár (Somogy) 1872 Lojka sub 51.

b. Ad sarmenta putrida Vitis viniferae. Kaposvár (Somogy) 5/1871 Lojka.

80. Lasiosphaeria Racodium (Pers. sub Sphaeria) Ces. et De N. syn: L. hirsuta (Fr. sub Sphaeria) Fuckel symb. myc. p. 147. c f r Karsten myc. fenn. II p. 161. Fuckel symb. myc. p. 147. Cooke hdb. p. 854 . Saccardo f. it. del. 587.

exs: Fuckel f. rhen. 950, 951. Saccardo myc. Ven. 779. Plowright sphaer. brit. II 53 (sub Sph. hirsuta). (? Rabh. hb. myc. II 649. f. eur. 829).

Speciminulum mancum! Asci clavati, 8 spori. Sporidia dactyloidea, obtusa, inferiore tertia parte subcurvata, 1 cellularia, plurinucleata, hyalina. 50/-5.

Ad lignum salicis. Kaposvár Lojka 1872 sub 200.

81. Lasiosphaeria hispida (Tode sub Sphaeria) Fuckel symb. myc. p. 147. f. terrestris (Sow.). Thümen.

c f r Saccardo f. it. del. 558. Cooke hdb. p. 855, 857.

exs: Fuckel f. rhen. 949 (? 2039 f. terrestris), Thümen myc. un. 1745 (? 1741 sub L. terrestris Thümen), ubi cit. syn. : L. hispida Fuckel symb. myc. p. 147 p. p. Sphaeria biformis Pers. syn. fung. 1. 56 p. p. Sphaeria terrestris Sow. f. tab. 373 f. 7.)

Asci clavati, 8 spori. Sporidia dactyloidea, apice superiore obtusa. inferiore acutata; inferiore tertia parte obtusangule curvata, 1 cellularia, nucleis quatuor magnis instructa, hyalina, disticha, 45/5. Pili simplices, septati, fusci, c. 4 mikr. crass.

Ad terram sylvae Kislakensis prope Kaposvár (Somogy). 1871 Lojka sub 198.

82. Leptospora ovina (Pers. sub Sphaeria) Ces. et De N.

s y n : Lasiosphaeria-Karsten myc. fenn. II p. 164.

ef $r^{\circ}$ Fuckel symb. myc. p. 143. Cooke hdb. p. 856. Saccardo f. it. del. 559. Michelia I p. 45 
exs: Rabh. hb. myc. II 730 (: f. eur. 1444). Fuckel f. rhen. 788. Thümen myc. un. 968. Plowright sphaer. brit. II 51 (? Cooke f. brit. II 565,576$)$.

Asci clavati, 8 spori. Sporidia cylindrica, obtusa, subcurvata, 1 cellularia, nucleis 2 magnis praedita, hyalina, disticha, 36/5. Paraphyses filiformes. Jod -

Ad truncum putridum in sylva Kislakensi prope Kaposvál (Somogy) in Hung. Lojka 1872 sub 205.

83. Enchnosphaeria Pinetorum Fuckel symb. myc. p. 147.

syn: Sphaeria scabra Curr.

cfr Cooke hdb. p. 859. Winter in Flora 1872 p. 510.

exs: Rehm Ascomyc. 43 (sub Bertia querceti Rehm), 90. Fuckel f. rhen. 1797. Rabh. f. eur. 1215 (sub Lasiosphaeria scabra Awd!

Asci clavati, 8 spori, 80/12. Sporidia fusoidea, obtusa, recta, medio constricta, 2 cellularia, quaque cellula nucleis 2 magnis praedita, hyalina, (interdum utraque apice breviter hyaline appendiculata), -306 , disticha. Paraphyses filiformes. Jod -

Ad basim peritheciorum hyphae fuscae, c. 4 mikr. crassae.

Ad caules putridas Aconiti alpis Dzurowa prope Teplicska (Liptó), alt. c. 5000`. 8/1872 Lnjka.

Verisimiliter exemplaria ad hanc speciem trahenda puto!

S4. Ciypeosphaeria Notarisii Fuckel symb. myc. p. 117. Nachtrag III p. 19.

syn: Sphaeria clypeiformis De $\mathrm{N}$. (? Sph. manillana Fr.) non $=$ Sph. Notarisii Car.

c f r Saccardo f. it. del. 189. Michelia I p. 39. II p. 65. Cooke hdb. p. 889 (sub Sph. clypeata Nees).

exs: Fuckel f. rhen. 1823 (sub Sordaria clypeata). Saccardo myc. Ven. 1159. Plowright sphaer. brit. I 85, III 57. (? Rabh. hb. myc. II 64ว).

Asci cylindracei, 8 spori, 1508 . Sporidia cylindracea, obtusa, 4 cellularia, plerumque quaque cellula $1-3$ nucleis praedita, demum ad septa subconstricta, fusca, monosticha - 24,6,5. Paraphyses tiliformes. Jodii ope apex ascorum coerulescit.

Ad ramum siccum Rosae in valle Kolczvárensi (Hunyad) Transsylvaniae. 8/1873 Lojka.

85. Sphaerella superflua (Awd sub Sphaeria) Fuckel symb. myc. p. 102. ef $r$ Cooke hdb. p. 9u7. Karsten myc. fenn. II p. 177. 
ex s: Fuckel f. rhen. 884. Plowright sphaer. brit. II 83 (? Kunze f. sel. 258 sub Didymosphaeria-Niessl).

Asci clavati, 8 spori. Sporidia oblongo-elliptica, subobtusa, recta vel subcurvata, 2 cellularia, hyalina, disticha, 15/4. Paraphyses desunt. Jod -

Ad caules putridas Umbelliferarum in alpe Retyezát (Hunyad) Transsylvaniae. 1873 Lojka.

86. Ophiobolus rubellus (Pers. sub Sphaeria) Rehm.

s y n: Rhaphidophora-Fuckel symb. myc p. 125.

c f r Karsten myc. fenn. II p. 165. Cooke hdb. p. 899. Saccardo myc. Ven. p. 101. Michelia II p. 67.

exs: Fuckel f. rhen. 787 (sub Leptospora). Rabh. f. eur. 1546 (sub Pleospora Doliolum), 1555 (sub Rh. erythrospora Oud.) (? hb. myc. II 532). Plowright sphaer. brit. II 77. Thümen myc. un. 561. Saccardo myc. Ven. 920. Kunze f. sel. 79, 254. Cooke f. brit. II 688. Ellis f. n. amer. 191. Rehm Ascomyc. 94.

Asci cylindracei, apice incrassati, 8 spori, 180/-5. Sporidia filiformia, multiseptata, flavidula, recta, parallela, 180/1,5. Paraphyses ramosae. Jod -

a. Ad caules putridas Umbelliferarum in horto Eszterházyano. Kaposvár (Somogy). 5/1871 Lojka.

Edita in Rehm Ascomyc. 94!

b. Ibidem ad ramulos putridos Sambuci Ebuli. 5/1871 Lojka.

c. Ad caules putridas Kitaibeliae vitifoliae. Ercsi (Fehér) in Hungaria. 9/1873 Lojka sub 2124.

d. Ad caules putridas Amaranthı. Lojka 1871 sub 201.

87. Ophiobolns Fruticum (Rob. sub Sphaeria) Rehm.

s y n: Rhaphidophora-Fuckel symb. myc. p. 125. Rh. Ononidis Awd.

ex s: Fuckel f. rhen. 779. Kunze f. sel. 80. Rehm Ascomyc. 140. Rabh. f. eur. 1256) (? 359). Thümen myc. un. 61 (? f. austr. 249).

Asci cylindraceo-clavati, apice incrassati, 8 spori, 120/12. Sporidia filiformia, subacuta, -20 cellularia, flavidula, vermicularia, $-120 / 4-5$, circumvoluta. Paraphyses ramosae. Jod -

Ad ramulos siccos Ononidis spinosae. Szent-Miklós in insula Csepel Danubii (Pest). 6/1873 Lojka sub 2001.

88. Ophiobolus compressus Rehm.

Perithecia dimidiata, nigra, hemisphaerico-conica, primitus sub epidermide nidulantia, dein sessilia, sicca compressa, seriatim aggregata, c. $0,5 \mathrm{~mm}$ diam, minutissime papillata poroque vix per- 
spicuo instructa, sicca utrimque compressa. Asci cylindraceo-clavati, 8 spori, 110/15. Sporidia filiformia, subacuta, medio subconstricta, 8-16 cellularia, cellulis nucleatis, flavescentia, parallela, 90/4. Paraphyses ramosae. Jod -

ex s: Rehm Ascomyc. 189 (sub Rhaphidophora).

Ad caules emortuas Artemisiae camp. prope Szent-Miklós in insula Danubii Csepel (Pest). 6/1873 Lojka sub 1992.

Edita in Rehm Ascomyc!

89. Ophiobolus Bardanae (Wallr. sub Sphaeria) Rehm.

s y n: Rhaphidophora-Fuckel symb. myc. p. 126.

exs: ? Fuckel f. rhen. 886 et Thümen f. austr. 475.

Asci cylindraceo-clavati, 8 spori, 1209 . Sporidia filiformia, multicellularia, subcurvata, circumvoluta, $90 /-3$; (hinc inde cellula una media sublatiore).

a. Ad caules putridas Angelicae sylv. infra alpem Retyezát (Hunyad) Transsylvaniae. 8/1873 Lojka.

b. Ad caules putridas Conii prope Kaposvár (Somogy). 1871 Lojka.

Nescio an exemplaria revera ad hanc speciem, mihi ipsa plane dubiam et forsitan ad Ophiobolum acuminatum ducendam, pertineant.

90. Sordaria fimicola (Rob. et. Desm. sub Sphaeria) Ces. et $\mathrm{De} N$.

s y n: Hypocopra fimeti b. equina Fuckel symb. myc. p. 241. Sordaria conferta Awd man. sec. Winter. Massaria fimeti Cooke hdb. p. 847 ; no n Ixodopsis-Karsten myc. fenn. II p. 50.

c fr Winter Sordarien p. 17 tab. VII f. 6 a-f. Niessl in Hedwigia 1873 p. 131 (non Michelia p. 221).

ex s: Fuckel f. rhen. 1802 (? 1001). Saccardo myc. Ven. 1181. Cooke f. brit. II 566 (? Kunze f. sel. 101). Rabh. hb. myc. II 433, f. eur. $644,830$.

Asci cylindracei, 8 spori. Sporidia elliptica, 1 cellularia, nucleo 1 magno centrali praedita, fusca, monosticha, $18-27-10$.

Ad stercus equinum in alpe Kralowa Hola (Iiptó). 9/1872 Lojka sub 1867 A.

Exemplaria vetusta quoad ascos et sporidia!

91. Sordaria bombardioides Awd.

c f r Winter Sord. p. 22 tab. VIII f. 11. Niessl Beiträge in Hedwigia 1873 p. 130.

Asci cylindracei, 8 spori. Sporidia obtuse elliptica, 1 cellularia, nucleo magno 1 centrali praedita, fusca, massa gelatinosa tenui involuta, monosticha, - 24.9. Paraphyses ramosae. Jod - 
Ad stercus leporinum. in sylva Toponárensi prope Kaposvár (Somogy) in Hung. 1872 Lojka sub 168.

92. Sordaria minuta Fuckel symb. myc. Nachtrag II p. 44.

syn: S. tetraspora Winter in Helwigia 1871 p. 161. (? S. coprophila Fuckel symb. myc. p. 244).

ex s: Rehm Ascomyc. 199. (? Rabh. f. eur. 1529 sub S. tetraspora).

Asci clavati, 8 spori. Sporidia ovata, 1 cellularia, nucleo 1 magno centrali praedita, fusca, altera apice in papillam hyalinam, altera in appendicem -- 30 mikr. long., 5 lat., hyalinam abeuntia, -17/9, disticha. sub 1890.

a. ad stercus vaccinum prope Lunkány in Hung. 7/1872 Lojka

b. ibidem. Malomviz (Hunyad) Transsylvaniae. 1872 Lojka sub 1877. neant!

Mihi dubium, an exemplaria ad speciem nominatam perti-

93. Sordaria fimiseda Ces. et De N.

syn: Cercophora-Fuckel symb. inyc. p. 245. Nachtrag II p. 326. Sphaeria lanuginosa Preuss. Arnium--Nke.

c fr Winter Sord. p. 25. tab. IX f. 13. Michelia I p. 226. Karsten myc. fenn. II p. 52.

exs: Rehm Ascomyc. 235 (non Fuckel f. rhen. $2037=$ Sordaria curvula De By).

Asci elliptico-clavati, crassi, -330/50, 8 spori. Sporidia elliptica vel ovoidea, primitus hyalina, dein fusca, 1 cellularia, nucleo 1 magno centrali praedita, $42-50 /-27$; altera apice appendiculo hyalino, striatulo, curvato, basi - 15 lat., long. 90 mikr., altera recto, c. 30/9 instructa, 1--2 sticha. Paraphyses ramosae. Jod -

Pili apicis perithecii breves, septati, fusci, c. 4 mikr. crassi.

Ad fimum vaccinum prope Lunkány et Fácset in Hungaria. 7/1872 Lojka sub 1890 et 1891.

Edita in Rehm Ascomyc!

94. Sordaria decipiens Winter.

c f r Fuckel symb. myc. Nachtrag II p. 44 f. 33. Michelia I p. 226.

Asci elliptico-clavati, 145 long., 8 spori. Sporidia ovoidea, 1 cellularia, nucleo centrali 1 magno praedita, fusca, 30/15; altera apice acutata appendiculo hyalino, striatulo $12 / 9$, altera apice truncata appendiculo filiformi, hyalino, c. 4.5 long., recto instructa. 
Ad fimum vaccinum prope Lunkány in Hungaria. 7/1872 Lojka sub 1889 et 1890 .

95. Sordaria curvula De By.

sy n : Sphaeria fimiseda De N. Cercophora conica Fuckel symb. myc. p. 245. Sordaria breviseta Karsten myc. fenn. I p. 52. Malinvernia breviseta Fuckel symb. myc. p. 243. Schizothecium fimicolum Cda. Sordaria appendiculata Awd. (non Ixidopsis fimicola (Rob. sub Sphaeria) Karsten myc. fenn. I p. 50. nec Cercophora fimiseda Fuckel symb. myc. p. 245).

ef $\mathrm{r}$ Winter Sordarien p. 37. tab. XI f. 22 cum f. coronata et aloides Winter ( $\mathrm{y} n$ : Sordaria-Fuckel symb. myc. Nachtrag II p. 43). Hedwigia 1873 p. 161, 1874 p. 54. Grevillea IV p. 113. Michelia II p. 26, 225.

ex s: Kunze f. sel. 102. Fuckel f. rhen. 2037. Saccardo myc. Ven. 1182. Plowright sphaer. brit. III 43. Rehm Ascomyc. 138, 200 (f. juvenilis). (? Cooke f. brit. II 682. Thümen f. austr. 1152. Fuckel f. rhen. 2549, 1002).

Asci elliptico-clavati, 8 spori, 120/21. Sporidia elliptica, fusca, 1 cellularia, nucleo centrali 1 magno instructa, altera apice in papillam hyalinam protracta, altera filiformiter, hyaline, 18/3 appendiculata, c. 27.12 (sine appendiculis!) disticha. Paraphyses ramosae. Jod -

a. Ad fimum vaccinum prope Lunkány in Hungaria. 1872 Lojka sub 1890.

b. ibidem in Galicia australi. 8/1 69 Lojka.

96. Sordaria coprophila (Fr. syst. sub Sphaeria) Ces. et De N.

syn: Hypoxylon - Fr. S. Veg. Cercophora mirabilis Fuckel symb. myc. p. 245.

c f $\mathrm{r}$ Winter Sordarien p. 26 tab. IX f. 14. Karsten myc. fenn. I p. 51. Michelia II p. 227. Cooke hdb. p. 866. Hedwigia 15 tab. 13.

exs: Fuckel f. rhen. 1057, 2271. Rabh. hb. myc. II 257. Spegazzini dec. it. 43 (? Rabh. f. eur. 830. Ellis n. am. f. 499. Sacc. myc. Ven. 1492). Rehm Ascomyc. 198, 234 (inevoluta!).

Sporidia cylindrica ut in Lasiosphaeria et tertia inferiore parte obtusangule curvata, 1 cellularia, nucleis 2 magnis praedita, hyalina, c. 42/6, 8 disticha et convoluta in ascis clavatis, apice incrassatis, c. 150/8. Paraphyses articulatae.

Exemplaria adhuc juvenilia, inevoluta!

a. Ad fimum vaccinum prope Fácset in Hung. $7 / 872$ Lojka. 
b. ibidem prope Malomviz (Hunyad) Transsylvaniae. 8/1872 Lojka.

Edita in Rehm Ascomyc. 234!

97. Sporormia minima Awd in Hedwigia 1868 p. 66.

c f r Fuckel symb. myc. p. 242. Karsten myc. fenn. I p. 110. Michelia III p. 230. Hedwigia 1878 p. 146. Grevillea VIII p. 108.

ex s: Fuckel f. rhen. 997 p. p. (sub Sph. fimetaria). Moug. et Nestler stirp. vog. 1169 (sub Sphaeria stercoris). Rabh. f. eur. 1339 a, b. Cooke f. brit. I 451, II 567 (?). Thümen f. austr. 1055. Kunze f. sel. 167 (sub Sp. intermedia). Saccardo myc. Ven. 1183, 1181, 1362 (?). Ellis n. am. f. 198. Rehm Ascomyc. 34.

Asci cylindraceo-clavati, 8 spori, 60/12. Sporidia oblonga, obtusa, 4 celluria, $24 / 4$, in 4 partes mox secendentia, mucore gelatinosa tenui circumdata, disticha. Paraphyses? Jod -

Ad fimum vaccinum. Gelsendorf (Stryj) in Galicia australi. Lojka.

98. Sporormia intermedia Awd in Hedwigia 1868 p. 66.

c f r Fuckel symb. myc. p. 242. Cooke hdb. p. 866. Michelia p. 230. Karsten myc. fenn. II p. 110. Hedwigia 1878 p. 146. (s y n? f. grandispora Spegazz. in Michelia I p. 230).

exs: Fuckel f. rhen. 997. p. p. Rehm Ascomyc. 134 (n o n Fuckel 903, Rabh. eur. 644).

Asci clavati, 8 spori, 150,30. Sporidia oblonga, 4 cellularia, $48,9-10$, mox in partes quatuor secedentia, fusca, disticha, mucore gelatinosa tenui circumdata. Paraphyses articulatae. Jod -

Ad fimum vaccinum in sylva Holobutowensi prope Brigidau (Sambor) Galiciae australis. 9/1869 Lojka.

99. Sphaerotheca Castagnei Lev. Fuckel symb. myc. p. 78.

f. Ac on iti.

Ascus 1 in Peridio. Sporidia plane inevoluta.

Ad caules putridas Aconiti Napelli infra alpem Retyezát (Hunyad) Transsylvaniae, alt. c. 6000'. 8/1873 Lojka.

100. Perisporium vulgare Corda.

syn: Preussia funiculata (Preuss sub Perisporium) Fuckel symb. myc. p. 91. Sporormia Fleischhackii Awd. Perisporium laeve Awd. (cfr Karsten myc. fenn. I p. 109). Preussia Kunzei Fuckel symb. myc. Nachtrag II p. 18. Perisporium Typharum Sacc. myc. Ven. p. 92.

c f r Sacc. f. Ven. V p. 174. Michelia p. 401. Cooke hdb. p. 
644. Saccardo f. it. del. 597-599. Hedwigia 1865 no 5 tab. I no 2, 1869 no 1 p. 2.

exs: Rabh. f. eur. 921, 1338, 1433. Fuckel f. rhen. 1750. Thümen myc. un. 161. Cooke f. brit. I 699 (f. telae).

Asci ovales, crassi, 8 spori. Sporidia oblonga, 4 cellularia, articulata et mox in partes quatuor soluta, parallela, 27/6, fusca. Paraphyses ramosae. Jod -

Ad lignum quercinum fabrefactum. Kaposvár (Somogy) 1872. Lojka sub 29.

\section{A d d e nda. $\left.{ }^{*}\right)$}

101. Lasiosphaeria canescens (Pers. sub Sphaeria) Karsten myc. fenn. II p. 162.

cf $r$ Cooke hdb. p. 858 (descriptio sporarum haud plane quadrat).

Ad lignum tecti. Árva-Varálja. in Hungaria. Lojka.

Ad 82: Le ptospora ovina (Pers).

Ad ramum siccum Sambuci racemosae in valle "Ticha dolina" (Liptó) Tátra Hung. Lojka sub 4273.

*) Aegre fero, me permulta, quae ad species supra descriptas addenda et in descriptione data corrigenda erant, nunc, quia hoc demum anno in lucem prodierunt, omittere coactum esse. 



\section{Index Specierum et Synonymorum.}

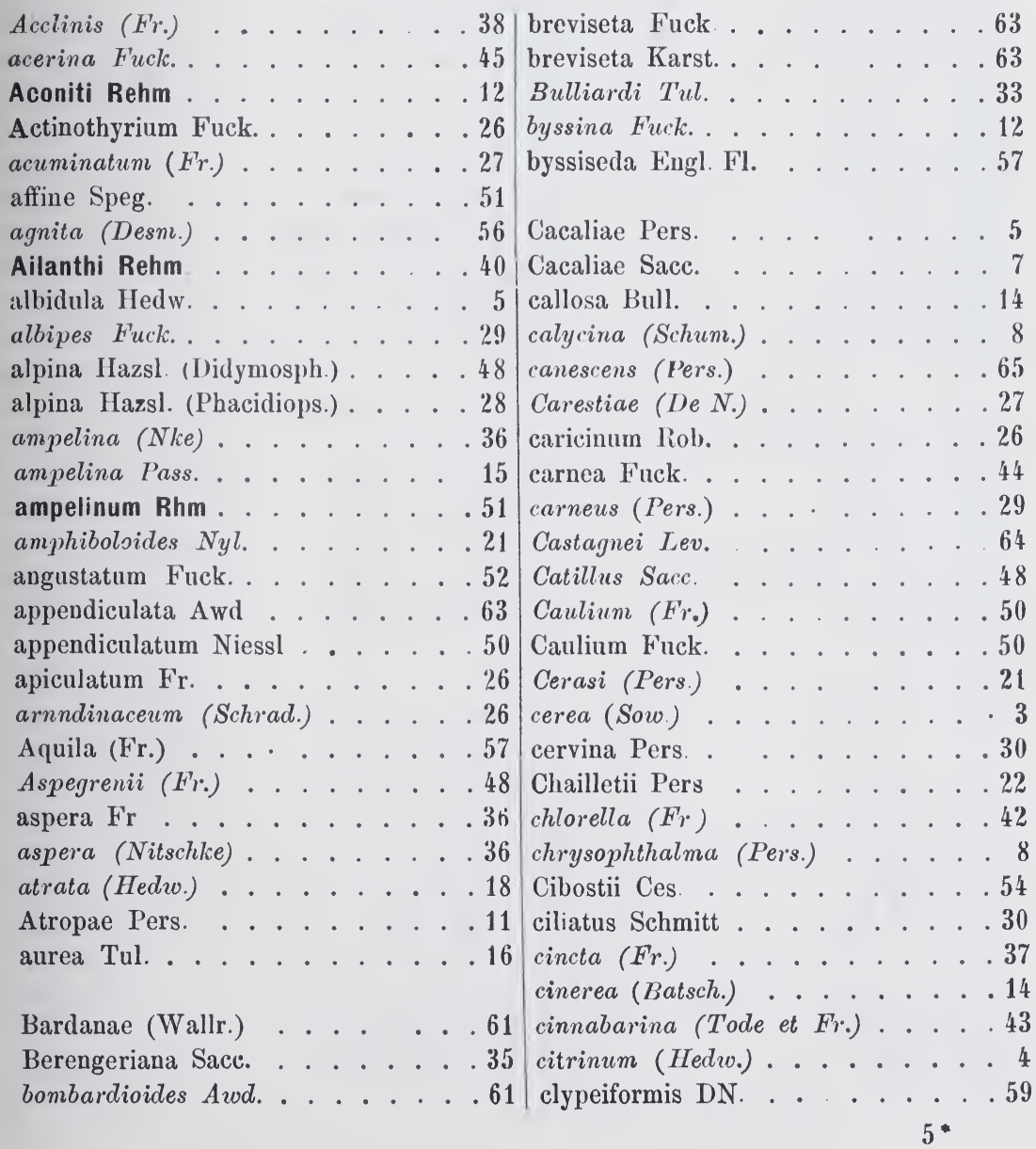


coccinea (Jacq.). . . . . . . . 4 coccinella Sommf. . . . . . 17 coerulescens Rehm . . . . . . 9 commutata Fuck. . . . . . . . . 19 compressum (Pers.) . . . . . . 52 compressus Rehm . . . . . . 60 conferta Awd. . . . . . . . 61 confluens. Tul. . . . . . . . 4 conica Fuck. . . . . . . . . . . 63 Coniothyrium (Fuck) . . . . . .56 conoidea Niessl . . . . . . . 52 convexellum (Karst.) . . . . . 8 coprinaria (Cooke) . . . . . . 1 coprophila (Fr.) . . . . . 63 coprophila Fuck. . . . . . . .62 coronata Niessl . . . . . . . 53 corrugatum Koerb. . . . . . . 46 corruscans Norm. . . . . . . 29 corticalis (Pers.) . . . . . . 23 crenatum (Pers.) . . . . . . . 51 cubicularis Fuck. . . . . . . . . 25 Cucurbitula (Tode) . . . . . . 42 culmigenum Fr. . . . . . . . . 26 curvula de By . . . . . . . . . . . 63 cyanogena Desm. . . . . . . 44 cyathoideum (Bull.) . . . . . . 5 Cypri (Tul.) . . . . . . . 38

Dacrymycella $\left(\mathrm{Nyl}_{\text {. }}\right)$. . . . . . 44 decipiens Wint........ . 62 decorticans (Fr.) . . . . . . 38 deflectens Karsten. . . . . . 52 depauperatus (Berk, et Br) . . . . 31 diaphana Rehm ........ . 16 dispersa D N. . . . . . . . 4' diversicolor Fr. . . . . . . . 30 Doliolum (Pers.) :. . . . . . . 55

Elegans Thümen ....... . 57 elongata (Fr.) . . . . . . 45 episphaerica (Tode) . . . . . 42 equina Müll. . . . . . . . . . 30 erythrinella Nyl. . . . . . . 44

Fascicularis (Alb. et Schw.) . . . 20 fimbriata Chaill. . . . . . . 22 Fimeti Cooke . . . . . . . 61 Fimeti Fuck. . . . . . . . 61 finicola (Rob. et Desm.) . . . . . 61

fimicolum Corda ...... . . 63

fimiseda Ces. et $D$ N. . . . . .62

fimiseda D N. . . . . . . 63

flacca (Wallr.) . . . . . . 44

flammea (Alb. et Schw.). . . . 23

flavorirens (Hoffm) . . . . . . 36

Fleischhackii Awd. . . . . . .64

Franzonianum De N. . . . . . 4

fructigenum (Bull.) . . . . 7

Fruticum (Rob.) . . . . . . 60

Fuckelii Duby . . . . . . 39

funiculata Fuck. . . . . . . .64

furfuraceus Pers. . . . . . . .31

fusarioides (Ber\%.) . . . . . 17

fusca (Pers.) . . . . . . . 12

fuscoatra Hazsl. . . . . . . . 22

fuscum (Pers.) . . . . . . . 33

Glaber Fuck. . . . . . . . . 31

glaber Karst. . . . . . . . . 31

glaciale Rehm . . . . . . . . 51

glanduliforme Rehm . . . . . 5

gramineum Moug. . . . . . . 26

granulatus Fuck. ........ . 2

granuliformis (Crouan) . . . . . 30

Herbarum (Pers.) . . . . . . 6

hirsuta Fr. . . . . . . . . . 58

hispida (Tode) . . . . . . . 58

Hoffmanni Tul . . . . . . 36

humilis Desm. . . . . . 6

Humuli (Lasch) . . . . . . . . . 6

Hungarica Rehm ....... . 13

Immersus Pers. . . . . . . . . . 32

inaequalis (Curr.) . . . . . . . 39

incrustans Nke...... . . 39

inquinans (Tode) . . . . . . . 57

insidiosum (Desm.) . . . . . . . 50

insitiva Ces. . . . . . . . . 34

intermedia Awd . . . . . . . 64

Iridis Rehm ......... . 16

Kalchbrenneri Fuck. . . . . . . . 44

Kerverni (Crouan) . . . . . . 30

Kerverni Karsten . . . . . . . 31 
Laeve Awd.

64 mytilinellum Karst. . . . . . 25

lageniforme Sollm. . . . . . 25

lanata Rehm ........ . 9

lanuginosa Preuss. . . . . 62

laricinum Fuck. . . . . . . . 22

Lecanactis Mass. . . . . . 18

lecanodes Ces........... 41

Lecanora Schm. et Kze . . . . . 16

lecideola (Fr.) . . . . . . . 19

lejoplaca (Fr.) . . . . . . . 36

lenticulare Fuck. ...... . 4

leptideum Fr. . . . . . . . . 24

leucomela DC ........ 22

leucophaea (Pers.) . . . . . . 9

leucostoma Rehm . . . . . . 10

lichenicola (Ces.) . . . . . . 44

lichenicola DN. . . . . . . 44

lichenicola Sacc. . . . . . . 44

lichenoides Rehm ....... . 47

Ligni (Desnı.) . . . . . . . 14

lignyota Fr. . . . . . . . 20

ligustici DC. . . . . . . 22

lineare (Fr.) . . . . . . . 27

lineare Rehm........ 26

lividofusca Fr. . . . . . . . 12

luctuosa Cooke ........ . 15

luteopallens (Nyl.) . . . . . 2

Lycii (Duby) . . . . . . . . 46

Macrospora (Fuck) . . . . . . . 57

macrospora Karst. . . . . . . 28

macrosporus Crouan . . . . . . 32

macrostomoides DN. . . . . . 50

macrostomum (Tode) . . . . . 49

mammillana Fr. . . . . . . . 54

Marianum Carus

mytilinum (Pers.)...... . 25

Napelli (Ces.) . . . . . . 40

nivea Fuck. . . . . . . . 11

Notarisii Fuck. . . . . . . . 59

nummularia DC. . . . . . . 34

Obesa Dur. . . . . . . . 54

ocellata Pers. . . . . . . . 16

omphalodes (Bull.) . . . . . 4

Ononidis Awd . . . . . . 60

Orni Rehm . . . . . . . 37

ovina (Pers.) . . . . . . 58, 65

Papillatus Wallr. ...... 30

parallela (Ach.) . . . . . . 28

parvula Speg. . . . . . . 16

Passerinii Sacc. . . . . . . 54

Patella (Tode) . . . . . . 22

patellaria Pers......... . 18

Peltigerae Fuck. . . . . . . . 20

Peltigerae Nyl. . . . . . . . 19

Peltigerae $\mathrm{Ph}$. et $\mathrm{Pl}$. . . . . 44

pertusum (Pers.) ....... . 48

perversum $D N$. . . . . . . 49

Peziza Tode .......... 41

pezizoides Zopf. . . . . . . 11

phaeotricha Rbh. . . . . . . 11

picastra Fuck. . . . . . . 47

pilosus (Fr.) . . . . . . . . . 30

Pinastri (Pers.). . . . . . . 23

Pinastri Tul........... 21

Pinetorum Fuck. . . . . . . . 59

pinicola (Rebent.) . . . . . . 28

pinicola Rehm . . . . . . . 48

Maydis Rehm . . . . . . . . 23

melaspermella Nyl. . . . . . 19

mimima Awd. . . . . . . . 64

mimima Fuck. . . . . . . . . 57

minuta Fuck.......... . .62

minutissima Rehm . . . . . . 42

minutula Koerb. . . . . . . 29

mirabilis Fuck. . . . . . . 63

modesta Desm. . . . . . . . 54

mollissima (Lasch.) . . . . . 10

moriformis (Tode) . . . . . . 46

Morthieri Fuck. . . . . . . . 47

pithyophila (Kze et $\mathrm{Fr}$.) . . . 45

plicata Rehm ......... 1 t

podoides Wallr.........35

Polygoni (Cooke) . . . . . 15

polymorpha Rehm .......57

Populi Lasch. . . . . . . 16

populnea Pers. . . . . . . 20

profusa $(F r$.) . . . . . 41

propolidoides Rehm ...... 24

Prunastri Fr. . . . . . . 21

Pruni Fuck. . . . . . . . 37

pseudomacrostomum Sacc. . . . 49 
pulicaris $(\mathrm{Fr}) \ldots . . . . .43,44$ punctata (L.) . . . . . . . . . 33 purpurea Rehm ......... . 54 pyrenopezizoides Speg. et Sacc. . . 55 pyrrhochlora $(A w d$.) . . . . . 41 Quadratus (Schmitt és $\dot{K} z e$ ) . . . . 24 quadrinucleatum Karst. . . . . . 49 quercinum (Pers) . . . . . 25

Racodium (Pers.) . . . . . . .58 relicina $(F r$. . . . . . . . 10 repanda (Wahlnb.) . . . . . 3 resinaria (Cooke et $\mathrm{Ph}$ ) . . . . . 11 Robergei Desm. ........ . 44 rosella Rehm . . . . . . . . 16 rubellus (Pers.) . . . . . . . 60 rubicunda Rehm ....... . 53 rubra Cooke . . . . . . . . . . 2 rubra Fuck. . . . . . . . . 17 rubricosum Fr. . . . . . . 35 rufum (Pers.) . . . . . . . 24

Saccharinus Boud..... . . 29 Sambuci (Pers.) . . . . . . . . 40 saprophila Fw. . . . . 18 Sarothanni Nle . . . . . . 39 Saubinetii (Dur. et Mont) . . . . 44 scabra Awd. . . . . . . . . 59 scabra Curr. . . . . . . . . 59 scotophila Sacc . . . . . . 53 Seiridii B. et Br. . . . . . . 41 seriatum Lib. . . . . . . . 26 setosa Niessl . . . . . . .54 simillimum Karst. . . . . . . 50 Solani Pers. . . . . . . . 5 spilomatica (Anzi) . . . . . . 29 spiniferum (Wallr.). . . . . . 35 spinosa (Pers.) . . . . . . . . 35 Stephensoniana Ellis .... . . 3 stercoraria Bull. ....... . 31 Stigma $(H n f f m$.$) . . . . . . 35$ striata Fr. . . . . . . 7
Strickeri Koerb. . . . . . . . . 19 subcompressum Karst. . . . . . 52 suecica Fuck . . . . . . . . . 9 sulphurea Fr. . . . . . . . . . 9 sulphurea Fuck. . . . . . . . 9 superflua (Awd) . . . . . . . 59 Syringae Nke . . . . . . . . 37

Syringae Rehm . . . . . . . 20

syringea Wallr......... 11

Tarzetta Cooke . . . . . . . 3

tenuis Fuck. . . . . . . . . 1

terrestris 'Thümen . . . . . . 58

tessera $\left(F^{\prime} r\right)$. . . . . . . 39

tetrasporum Fuck. . . . . . . 8

theleboloides (Alb. et Schw) . . . 1, 2 tortile (Schwe $n$.) . . . . . . . . 26

Transsylvanica Rehm . . . . . 47

Tyrolensis Rehm ....... 13

umbrata Cuoke . . . . . . . 3

umbrorum Fuck. . . . . . . . 2

unica Desm. . . . . . . . 25

unicaudata ( $B$ et $\mathrm{Br}$ ) . . . . . 52

Uiticae (Pers) ...... . 7

vagans Sacc. . . . . . 40

varians Hazsl. . . . . . . . 46

versicolor Karst . . . . . . . 31

vestitum $(\mathrm{Fr}$.) . . . . . . .40

vicinellum Sacc . . . . . . . 49

villosa (Pers.) . . . . . . . 11

vinosa (Alb. et Schw.) . . . . . . 17

vinosula Rehm . . . . . . 18

violascens Boud. . . . . . . . 31

Virgultorum $D C$. . . . . . 27

Virgultorum (Vahl.). . . . . . 7

Vitis Hazsl. . . . . . . . . 37

Vitis Rehm.......... . 5

vulgare Corda . . . . . . . 64

vulgaris $\mathrm{DN}$. . . . . . . 5

vulgaris Niessl ......... 53 



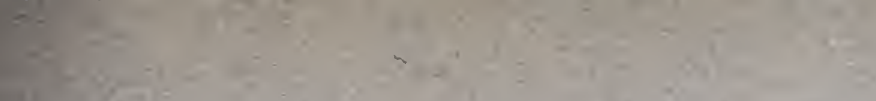

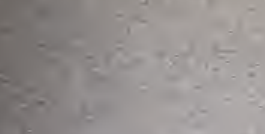

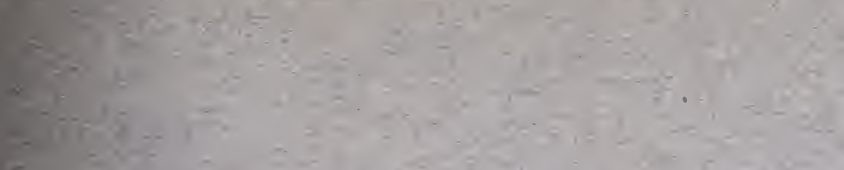

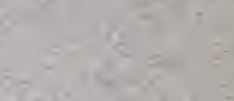

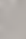

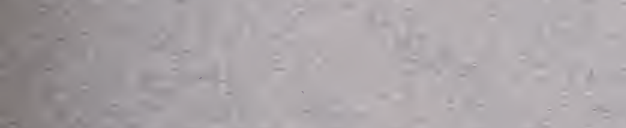

$8 x^{2}=-2$

20

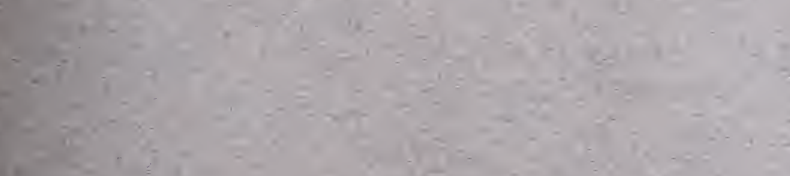

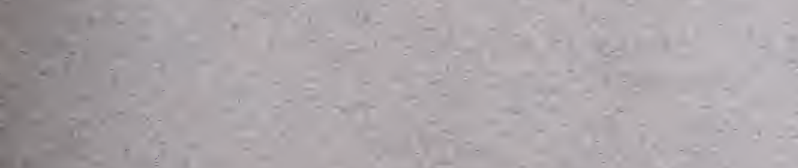

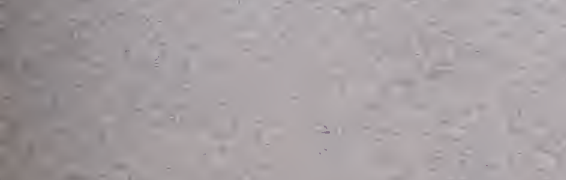



- 

